BARI-TH 431/02

\title{
EFFECTIVE DESCRIPTION AT VERY HIGH DENSITIES
}

\author{
Giuseppe Nardulli
}

Physics Department, University of Bari, Italy

I.N.F.N., Sezione di Bari, Italy

\begin{abstract}
We describe the effective lagrangian approach to the color superconductivity. Superconductivity in color interactions is due to vacuum instability at $T=0$ and high densities, which arises because the formation of quark-quark pairs is energetically favored. The true vacuum is therefore populated by a condensate of Cooper pairs breaking color and baryonic number. The effective langrangian follows from the Wilson's renormalization group approach and is based on the idea that at $T=0$ and $\mu \rightarrow \infty$ the only relevant degrees of freedom are those near the Fermi surface. The effective description that arises if one considers only the leading terms in the $1 / \mu$ expansion is particularly simple. It is based on a lagrangian whose effective fermion fields are velocity-dependent; moreover strong interactions do not change quark velocity (Fermi velocity superselection rule) and the effective lagrangian does not contain spin matrices. All these features render the effective theory similar to the Heavy Quark Effective Theory, which is the limit of Quantum ChromoDynamics for $m_{Q} \rightarrow \infty$. For this reason one can refer to the effective lagrangian at high density as the High Density Effective Theory (HDET). In some cases HDET results in analytical, though approximate, relations that are particularly simple to handle.

After a pedagogical introduction, several topics are considered. They include the treatment of the Color-Flavor-Locking and the 2SC model, with evaluation of the gap parameters by the Nambu-Gorkov equations, approximate dispersion laws for the gluons and calculations of the Nambu-Goldstone Bosons properties. We also discuss the effective lagrangian for the crystalline color superconductive (LOFF) phase and we give a description of the phonon field related to the breaking of the rotational and translational invariance. Finally a few astrophysical applications of color superconductivity are discussed.
\end{abstract}





\section{Contents}

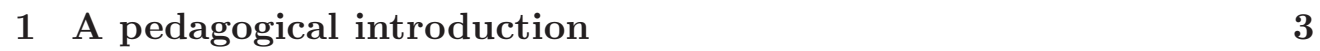

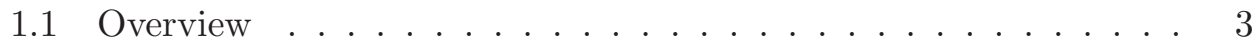

1.2 Color superconductivity $\ldots \ldots \ldots$. . . . . . . . . . . 6

1.3 Effective action à la Wilson . . . . . . . . . . . . . . . . . . . 8

1.4 The true vacuum . . . . . . . . . . . . . . . . . . 13

1.5 Effective lagrangian for the NGB in the CFL mode $\ldots . . . .16$

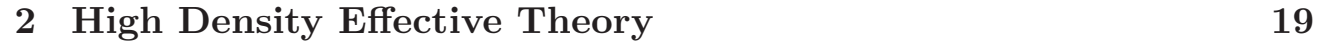

2.1 The 2-D effective theory . . . . . . . . . . . . . . . . . . . 19

2.2 HDET for superconducting phases . . . . . . . . . . . . . . 23

2.2 .1 HDET in the CFL phase . . . . . . . . . . . . 24

2.2 .2 HDET in the 2 SC phase . . . . . . . . . . . . . . . . . . . . . . . 25

2.3 NGB and their parameters . . . . . . . . . . . . . 27

2.3.1 Gradient expansion for the $U(1)$ NGB in the CFL mode] and in the 2SC model . . . . . . . . . . . 27

$2.3 .2 \quad$ Gradient expansion for the $S U(3)$ NGBS . . . . . . . . . . 31

2.4 Gluon masses and dispersion laws . . . . . . . . . . . . . . . . 33

2.4 .1 Evaluating the bare gluon mass . . . . . . . . . . . . . 33

2.4 .2 Debye and Meissner mass and gluon approximate dispersion laws: CFL cast . . . . . . . . . . . . 34

2.4.3 Debye and Meissner mass and gluon approximate dispersion laws: 2 SC case $\ldots \ldots \ldots \ldots$. . . . . . . . . . . 39

2.5 One-loop corrections to three- and four-point gluon vertices in the 2 SC mode $\ldots \ldots \ldots \ldots \ldots . \ldots \ldots 4$

2.6 Gap equation in HDET . . . . . . . . . . . . . . . . . . 45

2.7 Appendix. A few useful integrals . . . . . . . . . . . . 47

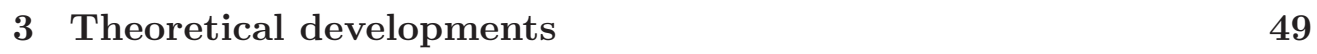

3.1 Advances in the CFL model . . . . . . . . . . . . . . . . . . . . . 49

3.1.1 $\quad$ Would-be NGBs masses . . . . . . . . . . . . . . . . . . . 49

3.1 .2 Electromagnetic interactions . . . . . . . . . . 50

3.1 .3 Quark-hadron continuity . . . . . . . . . . . . . . . 51

3.1 .4 Asymptotic analysis . . . . . . . . . . . . . . . . 52

3.2 Other models . . . . . . . . . . . . . . . 53

3.2 .1 One flavor . . . . . . . . . . . . . . . . . . . 53

3.2 .2 QCD at large isospin density . . . . . . . . . . . 54 
$3.3 \quad$ Transport theory for quarks in dense matter . . . . . . . . . . . 54

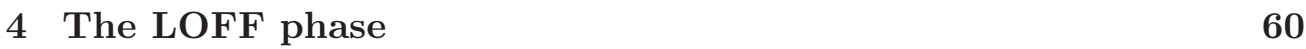

4.1 Overview of the crystalline superconductive (LOFF) state . . . . 60

4.2 Phonons in the LOFF phase . . . . . . . . . . . . . . 62

4.3 HDET approach to the LOFF state . . . . . . . . . . . . 63

4.4 Phonon-quark coupling . . . . . . . . . . . . . . . . . . . . . . . . 68

4.5 Dispersion law for the phonon field . . . . . . . . . . . . . . 69

$\begin{array}{lll}5 & \text { Astrophysical implications } & \mathbf{7 0}\end{array}$

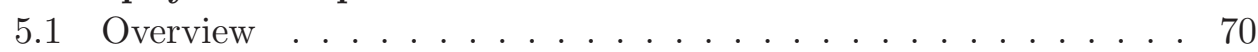

$5.2 \quad$ A brief introduction to compact star . . . . . . . . . . . . 71

5.3 Supernovae neutrinos and cooling of neutron stars . . . . . . . . 75

$5.4 \quad R$-mode instabilities in neutron stars and strange stars . . . . . . 76

5.5 Miscellaneous results . . . . . . . . . . . . . . 77

$5.6 \quad$ Glitches in neutron stars . . . . . . . . . . . . . . . . . 78 


\section{Chapter 1}

\section{A pedagogical introduction}

\subsection{Overview}

The aim of the present review is to describe various approaches to the color superconductivity (CSC) [1] that are based on the method of the effective lagrangians. CSC is one of the most fascinating advances in Quantum Chromo Dynamics (QCD) in recent years [2, 3, 4, 5, 6, 7, 8, 9, 10, 11, 12, 13] (for reviews see [14, 15, 16]); it offers a clue to the behavior of strong interactions at very high baryonic densities, an issue of paramount relevance both for the understanding of heavy ion collisions and the physics of compact stars.

The origin of the superconductivity in color interactions is related to the instability of the naive vacuum at $T=0$; at low densities this instability is supposed to arise because the formation of a quark-antiquark pair is energetically favored; therefore Cooper pairs formed by a quark and an antiquark (in a color singlet representation) populate the true vacuum. This phase is indeed characterized by the order parameter

$$
\langle\bar{\psi} \psi\rangle \neq 0
$$

i.e. by a condensate of $\bar{q} q$ pairs, similarly to the phenomenon of the Cooper pairs in ordinary superconducting materials at low temperatures, described by the theory of Bardeen, Cooper and Schrieffer (BCS) 17]. The condensate (1.1) violates chiral invariance, which is a symmetry of the QCD Lagrangian if one neglects the current quark masses. Therefore a non vanishing condensate (1.1) is tantamount to the spontaneous breaking of the chiral symmetry and the existence, because of the Goldstone's theorem, of a certain number of massless particles, the Nambu-Goldstone-Bosons (NGBs).

At high baryonic densities a different phenomenon sets in. As a matter of fact, for sufficiently high baryon chemical potential $\mu$, the color interaction favors the formation of a quark-quark condensate in the color antisymmetric channel. This condensate

$$
\left\langle\psi_{i \alpha}^{T} C \psi_{j \beta}\right\rangle
$$

( $\alpha, \beta=1,2,3$ color indices; $i, j=1,2,3$ flavor indices) acts as the order parameter of a new phase where both the $S U(3)_{c}$ color symmetry and the $U(1)_{B}$ baryonic number are spontaneously broken. As to chiral symmetry, the color 
interaction favors in general pairing of quarks of total spin 0 and opposite momenta; therefore the quarks in (1.2) have the same helicities and the condensate (1.2) does not break chiral invariance explicitly円.

Different phenomena take place depending on the value of the order parameter (1.2). One could have indeed:

$$
\epsilon^{\alpha \beta \gamma} \epsilon_{i j}<\psi_{i \alpha}^{T} C \psi_{j \beta}>=\Delta \delta^{\gamma 3}
$$

where the sum over the flavor indices run from 1 to 2 and $\psi$ represents a left handed 2-component Weyl spinor (the right handed field satisfies the same relation with $\Delta \rightarrow-\Delta$ ); moreover a sum over spinor indices is understood and $C=i \sigma_{2}$. This case correspond to the decoupling of the strange quark $\left(m_{s} \rightarrow\right.$ $\left.\infty ; m_{u}=m_{d}=0\right)$ and is called in the literature 2SC model. From dynamical analyses [2], [14] one knows that, for $\mu$ sufficiently large, the condensate (1.3) is non vanishing. Therefore it breaks the original symmetry group $S U(3)_{c} \otimes$ $S U(2)_{L} \otimes S U(2)_{R} \otimes U(1)_{B}$ down to

$$
S U(2)_{c} \otimes S U(2)_{L} \otimes S U(2)_{R} \otimes Z_{2}
$$

The chiral group remains unbroken, while the original color symmetry group is broken to $S U(2)_{c}$, with generators $T^{A}$ corresponding to the generators $T^{1}, T^{2}, T^{3}$ of $S U(3)_{c}$. As a consequence, three gluons remain massless whereas the remaining five acquire a mass. The $Z_{2}$ group means that the quark fields can still be multiplied by -1 . Even though the original $U(1)_{B}$ is broken there is an unbroken global symmetry that plays the role of $U(1)_{B}$. As for $U(1)_{A}$, this axial symmetry is broken by anomalies, so that in principle there is no Goldstone boson associated to its breaking by the condensate; however at high densities explicit axial symmetry breaking is weak and therefore there is a light would be Goldstone boson associated to the breaking of the axial color. One can construct an effective theory to describe the emergence of the unbroken subgroup $S U(2)_{c}$ and the low energy excitations, much in the same way as one builds up chiral effective lagrangian with effective fields at zero density. For the two flavor case this development can be found in [19].

For the three flavor case $\left(m_{u}=m_{d}=m_{s}=0\right)$ the following interesting case has been widely discussed [3]:

$$
\left\langle\psi_{i \alpha}^{L} \psi_{j \beta}^{L}\right\rangle=-\left\langle\psi_{i \alpha}^{R} \psi_{j \beta}^{R}\right\rangle=\Delta \sum_{K=1}^{3} \epsilon_{\alpha \beta K} \epsilon_{i j K} .
$$

The condensate (1.5) breaks the original symmetry group $S U(3)_{c} \otimes S U(3)_{L} \otimes$ $S U(3)_{R} \otimes U(1)_{B}$ down to

$$
S U(3)_{c+L+R} \otimes Z_{2}
$$

\footnotetext{
${ }^{1}$ The presence of an external chromagnetic field may affect CSC: for an analysis see [18].

${ }^{2}$ The more general case is

$$
\left\langle\psi_{i \alpha}^{L} \psi_{j \beta}^{L}\right\rangle=-\left\langle\psi_{i \alpha}^{R} \psi_{j \beta}^{R}\right\rangle=\gamma_{1} \delta_{a i} \delta_{b j}+\gamma_{2} \delta_{a j} \delta_{b i},
$$
}

but the solution with $\gamma_{1}=-\gamma_{2}$ corresponding to (1.5) is energetically favored. 
Both the chiral group and the color symmetry are broken but a diagonal $S U(3)$ subgroup remains unbroken in a way that locks together color and flavor (ColorFlavor-Locking=CFL model). We have 17 broken generators; since there is a broken gauge group, 8 of these generators correspond to 8 longitudinal degrees of the gluons, because the gauge bosons acquire a mass; one has 9 NGBs organized in an octet associated to the breaking of the flavor group and in a singlet associated to the breaking of the baryonic number. The effective theory describing the NGB for the CFL model has been studied in [20].

The 2SC model might be applicable for intermediate chemical potential $\mu$, while for very high $\mu$ the CFL phase should set in; therefore the QCD phase diagram should display different phases as schematically depicted in Fig. 1.1.

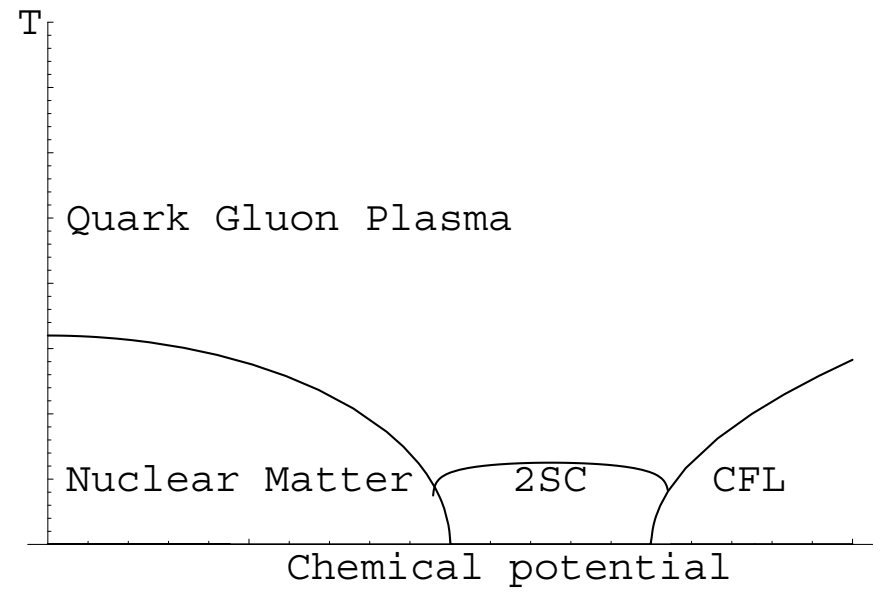

Fig. 1.1 Schematic view of the QCD phase diagram

Similarly to the chiral symmetry breaking condensate (1.1), the phase transition and the non vanishing of the condensates (1.3) or (1.5) result from a mechanism analogous to the formation of an electron Cooper pair in a BCS superconductor. At $T=0$ the only QCD interactions are those involving fermions near the Fermi surface. Quarks inside the Fermi sphere cannot interact because of the Pauli principle, unless the interactions involve large momentum exchanges. In this way the quarks can escape the Fermi surface, but these processes are disfavored, as large momentum transfers imply small couplings due to the asymptotic freedom property of QCD. Even though interactions of fermions near the Fermi surface involve momenta of the order of $\mu$, their effects are not necessarily negligible. As a matter of fact, even a small attractive interaction between fermions near the Fermi surface and carrying opposite momenta can create an instability and give rise to coherent effects. This is what really happens [2], [3] and the result is the formation of a diquark condensate, as expressed by (1.3) or (1.5). We stress again that the only relevant fermion degrees of freedom are therefore those near the Fermi surface.

This property and the effective theory for the fields at the Fermi surface is discussed for electromagnetism and ordinary BCS superconductivity in [21, 22, 23] where the Wilson Renormalization Group approach [24] is used to prove 
the Landau Theory of the Fermi liquid [25] and to prove the marginal character of the BCS interaction. First works towards the implementation of these ideas in the context of color superconductivity can be found in [26, 27, 28]. In 29] this approach was used to build up an effective theory for the CFL model based on the approximation of the neglect of the negative energy states. This results in a rather terse formalism resembling the heavy quark effective theory [30] and displaying as a characteristic note the existence of a Fermi velocity superselection rule and effective velocity-dependent fermion fields. Due to these features, we refer to this effective lagrangian as the High Density Effective Theory (HDET). In [31] the 2SC model has been studied by the same formalism, while in [32, 33, 34] this effective theory has been applied to a different color superconducting phase, the crystalline color superconducting phase [35, 36, 37, 38, 39], also called the LOFF phase from the authors of the original model of the crystalline electromagnetic superconductivity [40].

The aim of this paper is to review the developments in the description of Color Super Conductivity that are based on the effective lagrangian approach, in particular the HDET approach. While there are good reviews of the whole subject, the approach based on effective lagrangian has not been extensively discussed yet and this paper aims to do that. In the remaining part of this first section we will present an elementary introduction to some basic aspects of color superconductivity, most notably the Wilsonian version of the Landau theory of Fermi liquids and the derivation of the gap equation. The effective lagrangian approach for the two basic models (CFL and 2SC) is discussed extensively in the subsequent Section 2. In Section 3 we review some theoretical developments that in a way or in another are related to the main theme of this survey. In Section 4 we present a detailed discussion of the LOFF state of QCD. Finally in Section 5 we discuss astrophysical implications of color superconductivity in the context of the compact stars phenomenology.

\subsection{Color superconductivity}

To start with, we consider a non interacting fermion gas; its energy distribution $(\beta=1 / k T)$

$$
f(E)=\frac{1}{e^{\beta(E-\mu)}+1},
$$

becomes, when the gas is degenerate $(T \rightarrow 0)$, the step function, see fig. 1.2:

$$
f(E) \rightarrow \theta(\mu-E)
$$

The condition $E \leq \mu$ defines a domain in the momentum space whose boundary is the surface $E(p) \equiv \epsilon_{F}=\mu$, called Fermi surface; $\epsilon_{F}$ is called Fermi energy and the momentum $p_{F}$ satisfying $E\left(p_{F}\right)=\mu$ is the Fermi momentum. Any quantum state inside the Fermi surface, i.e. $E(p) \leq \epsilon_{F}$ is occupied by a fermion, and the other ones are empty. For $m=0, E=p$ and the Fermi surface is spherical $p_{x}^{2}+p_{y}^{2}+p_{z}^{2}=p_{F}^{2}=\mu^{2}$. This results applies also for non relativistic non interacting fermion (with $p_{F}^{2}=2 m \mu$ ). 
In presence of interactions the situation is more complicated, because, in general $f=f(\vec{r}, \vec{p})$. At very large chemical potentials one can however take advantage of the asymptotic (large $p$ ) properties of QCD, which implies that at $T=0$ the only QCD interactions are those involving fermions near the Fermi surface, as we remarked already. We shall discuss in the sequel the role of the surviving interactions for the quarks at the Fermi surface that, even if small, can be not negligible. For the time being we observe that the chemical potential is related to the fermion density (number of fermions per unit of volume $n$ by

$$
n=g \int \frac{d^{3} p}{(2 \pi \hbar)^{3}} f,
$$

where $g$ is the degeneracy of the state. If $f(\vec{r}, \overrightarrow{, p})$ does not depend on $\vec{r}$ this results in a constant density $n$. Clearly the density increases with $\mu$; in particular, for degenerate free fermions one gets

$$
n=\frac{4 \pi g}{(2 \pi \hbar)^{3}} \int_{0}^{p_{F}} p^{2} d p=\frac{4 \pi g p_{F}^{3}}{3 h^{3}} .
$$

In a similar vein the energy density and the pressure are given by:

$$
\epsilon=g \int \frac{d^{3} p}{(2 \pi \hbar)^{3}} E f
$$

and

$$
P=g \int \frac{d^{3} p}{(2 \pi \hbar)^{3}} \frac{p v}{3} f
$$

respectively. For non interacting fermions the state we have described is the

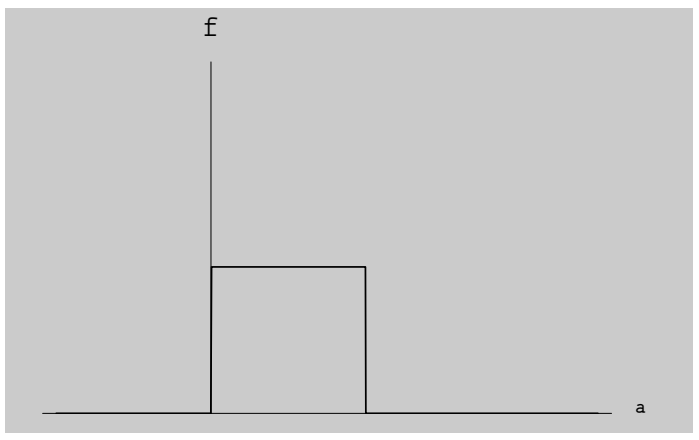

Fig. 1.2 Fermi distribution $f(E)$ at $T=0 . f(E)=0$ for $E>\mu=\epsilon_{F}$, and $f(E)=1$ for $0 \leq E \leq \mu=\epsilon_{F}$.

ground-state, i.e. the vacuum. Let us now suppose that the fermions are quarks and let us discuss the origin of the color superconductivity; we show by a simple argument that the naive vacuum is unstable provided an attractive interaction exists. Let us consider a pair of non interacting fermions near the Fermi surface; their energy is $\approx 2 \mu$. Let us substitute them with a bound state, i.e. a pair (a boson) with binding energy $\epsilon_{\text {int }}<0$. The fermion energy decreases by the amount $-2 \mu$; on the other hand the boson energy increases by $2 \mu+\epsilon_{\text {int }}$, so that the total energy is decreased by the amount $-\left|\epsilon_{\text {int }}\right|$. Therefore the formation of 
a bound state is favored. This might appear a small thing, but it is not, as one can immediately realize since there is an infinite number of ways to do that. The Cooper pair has preferably total momentum and total spin zero, but there is an infinite number of possible pairs, corresponding to the different directions of the Fermi velocity, see fig. 1.3

$$
\vec{v}_{F}=\frac{\vec{p}_{F}}{\mu}
$$

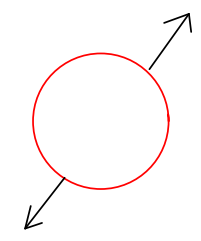

Fig. 1.3 Cooper pair of two fermions living at the Fermi surface with total momentum and total spin zero.

For the phenomenon of the superconductivity to occur one therefore needs an attractive interaction between the fermions. In the case of electronic superconductivity in the metals the attraction is the effect of the interaction of the electrons with the phonon: the resulting interaction between the fermions is indeed attractive and provides the required binding energy of the electronic Cooper pair. In the case of quarks the situation is more favorable because the QCD interaction in the color antitriplet $\overline{\mathbf{3}}$ (antisymmetric color channel) is attractive; therefore color superconductivity can occur even in absence of a lattice. The result of the Cooper interaction is the formation of a colored diquark condensate, with total baryonic number 2 .

The possibility of color superconductivity was studied long ago [1], but only recently [2] it has been realized that the effect offers several physical possibilities and is indeed sufficiently robust to survive the thermal fluctuations. The physical mechanism at its basis arises from interactions between two fermions at the Fermi surface. They will be discussed in the next paragraph.

\subsection{Effective action à la Wilson}

The previous results can be expressed in a more formal way by making use of the Landau theory of the Fermi liquid [25]; we will describe it in the Renormalization Group language à la Wilson [21, 22, 23, 24] ․ The idea of this approach is that the effective interactions of the fermions at $T=0$ can be described substituting the particles with their excitations, the quasiparticles that are in one-to-one correspondence with the particles. If one is interested only in

\footnotetext{
${ }^{3}$ Our presentation follows [21]; a similar discussion is in 26].
} 
special values of the momenta (in our case momenta near the Fermi surface), one can ignore the details of the interaction, which considerably simplifies the description without loss of physical insight. The simplification consists in the fact that the quasiparticles can be treated as free. However the form of the energy dependence on the momentum, i.e. the dispersion law $\epsilon=\epsilon(\vec{p})$, differs from the free particle case.

To derive in the framework of the Landau theory the results already discussed in previous sections, let us start with a gas of fermions at $T \simeq 0$ and the free lagrangian

$$
\mathcal{L}=\bar{\psi}\left(i \not \partial+\mu \gamma_{0}\right) \psi
$$

from which the free action can be constructed

$$
S=\int d t d \vec{p} \psi^{\dagger}(\vec{p})\left(i \partial_{t}+\mu-\epsilon(\vec{p})\right) \psi(\vec{p})
$$

For free particles one obtains (1.13) using the Dirac equation to write $\vec{\alpha} \cdot \vec{p} \psi=$ $\gamma_{0} \vec{\gamma} \cdot \vec{p} \psi=\epsilon(\vec{p}) \psi$ (see below, Eq. (2.1)); therefore, for free particles, $\epsilon(\vec{p})=$ $\sqrt{\vec{p}^{2}+m^{2}}$. However the interactions can modify this simple dispersion law and therefore we do not use this result. Because of this possible modifications, as we have already stressed, the particles are substituted, in the Landau theory of the normal Fermi liquid, by quasi-particles, that we can imagine as particles dressed by their interactions.

Let us now consider what happens when we scale energies and momenta down towards the Fermi surface. Let us divide the fermion momentum as follows

$$
\vec{p}=\vec{k}+\vec{\ell},
$$

with $\vec{k}$ on the Fermi surface and $\epsilon(\vec{k})=\mu$ (i.e. $\vec{k}$ is a Fermi momentum), while $\vec{\ell}=\ell \vec{n}$, with $\vec{n}$ a unit vector orthogonal to the Fermi surface. We scale down the momenta by a factor $s \rightarrow 0$ so that they approach the Fermi surface; clearly

$$
\begin{aligned}
& E \rightarrow s E, \\
& \vec{k} \rightarrow \vec{k}, \\
& \vec{\ell} \rightarrow s \vec{\ell} .
\end{aligned}
$$

We note that these conditions can be also expressed as follows:

$$
E,|\vec{\ell}|<\delta \text {. }
$$

with $\delta \leq \mu$. Moreover

$$
\epsilon(\vec{p})-\mu \rightarrow \epsilon(\vec{k})-\mu+\left.(\vec{p}-\vec{k}) \cdot \frac{\partial \epsilon}{\partial \vec{p}}\right|_{\vec{p}=\vec{k}}=\left.\vec{\ell} \cdot \frac{\partial \epsilon}{\partial \vec{p}}\right|_{\vec{p}=\vec{k}}=\ell v_{F}(k),
$$

where

$$
\vec{v}_{F}(\vec{k})=\left.\frac{\partial \epsilon}{\partial \vec{p}}\right|_{\vec{p}=\vec{k}}
$$

is a vector orthogonal to the Fermi surface. Therefore

$$
S=\int d t d \vec{k} d \ell \psi^{\dagger}(\vec{p})\left(i \partial_{t}-\ell v_{F}(\vec{k})\right) \psi(\vec{p})
$$


Under the scale transformation (1.15) we also have

$$
\begin{array}{ccc}
d t \rightarrow s^{-1} d t, & d^{3} \vec{p}=d^{2} \vec{k} d \ell \rightarrow s d^{2} \vec{k} d \ell, \\
\partial_{t} \rightarrow s \partial_{t}, & \ell \rightarrow s \ell,
\end{array}
$$

which means that the fields $\psi$ in the action $S$ of Eq. (1.13) scale as $s^{-1 / 2}$, while $S$ scales as $s^{0}$. Let us note explicitly that for spherical Fermi surfaces the integration is 2-dimensional and the measure is

$$
d \vec{k}=p_{F}^{2} d \Omega
$$

Let us now consider the terms in the action that might result from the interactions; they are constrained by the symmetries of the problem. We classify them according to the number of fermion fields:

1. A term quadratic in the fermion fields, such as

$$
\int d t d^{2} \vec{k} d \ell m(\vec{k}) \psi^{\dagger}(\vec{p}) \psi(\vec{p})
$$

scales as $s^{-1}$. Potentially this is a relevant operator 1 . However $m(\vec{p})$ can be absorbed 5 in the definition of $\epsilon(\vec{p})$, so that (1.22) is not a new operator at all. As to other quadratic terms such as, for example, those containing time derivatives and/or factor of $\ell$ they can be either absorbed in the corresponding terms in $(1.13)$ or are irrelevant.

2. A quartic term such as

$$
\begin{gathered}
\int d t \quad \prod_{j=1}^{4} \quad\left(d^{2} \vec{k}_{j} d \ell_{j}\right)\left(\psi^{\dagger}\left(\vec{p}_{1}\right) \psi\left(\vec{p}_{3}\right)\right)\left(\psi^{\dagger}\left(\vec{p}_{2}\right) \psi\left(\vec{p}_{4}\right)\right) \\
\times \quad V\left(\left\{\vec{k}_{j}\right\}\right) \delta\left(\vec{p}_{1}+\vec{p}_{2}-\vec{p}_{3}-\vec{p}_{4}\right)
\end{gathered}
$$

scales as $s=s^{-1} s^{-4 / 2+4}$ multiplied by the scale factor of the Dirac delta; it is therefore irrelevant if one can assume that

$$
\begin{aligned}
\delta\left(\vec{p}_{1}+\vec{p}_{2}-\vec{p}_{3}-\vec{p}_{4}\right) & =\delta\left(\vec{k}_{1}+\vec{\ell}_{1}+\vec{k}_{2}+\vec{\ell}_{2}-\vec{k}_{3}-\vec{\ell}_{3}-\vec{k}_{4}-\vec{\ell}_{4}\right) \\
& \rightarrow \delta\left(\vec{k}_{1}+\vec{k}_{2}-\vec{k}_{3}-\vec{k}_{4}\right) .
\end{aligned}
$$

3. Operators containing $2 n(n>2)$ fermion fields scale as $s^{n-1}$ and are therefore irrelevant.

\footnotetext{
${ }^{4}$ In the language of the Renormalization Group, an operator that becomes less and less important when the momenta scale is called irrelevant; an operator that becomes more and more important is called relevant; an operator equally important at all scales is called marginal.

${ }^{5}$ Since we can absorb $m(\vec{p})$ into $\epsilon(\vec{p})$ we cannot assume for the dispersion law the free particle expression; the specific form of the dispersion law depends on the nature of the interaction.
} 
This is the Landau theory of the normal Fermi liquid derived by the renormalization group approach. Let us now explain how this theory is modified for superconducting materials. Let us consider an interaction term with a quartic coupling (1.23) such that the two incoming momenta $\vec{p}_{1}$ and $\vec{p}_{2}$ satisfy $\vec{p}_{1}+\vec{p}_{2}=0$ (see Fig. 1.3). If the Fermi surface is parity symmetric, which we assume, then $\vec{k}_{1}+\vec{k}_{2}=0$ and also $\vec{k}_{3}+\vec{k}_{4}=0$. In other terms, for the special case of scattering with zero momentum, instead of (1.24) we have

$$
\delta\left(\vec{p}_{1}+\vec{p}_{2}-\vec{p}_{3}-\vec{p}_{4}\right)=\delta\left(\vec{\ell}_{1}+\vec{\ell}_{2}-\vec{\ell}_{3}-\vec{\ell}_{4}\right),
$$

which scales as $s^{-1}$.

It follows that the corresponding operator becomes marginal and, since there are no relevant interactions, it may dominate. This is the mechanism at the basis of the phenomenon of Cooper pairing in the BCS 5 superconductors. The Cooper pairing dominates over the electronic electrostatic repulsion, in spite of the fact that the Cooper pair has a size of $10^{4} \AA$ and the binding energy between the electrons is of order $10^{-3} \mathrm{eV}$, while typical electronic interactions are of the order of the electron volt.
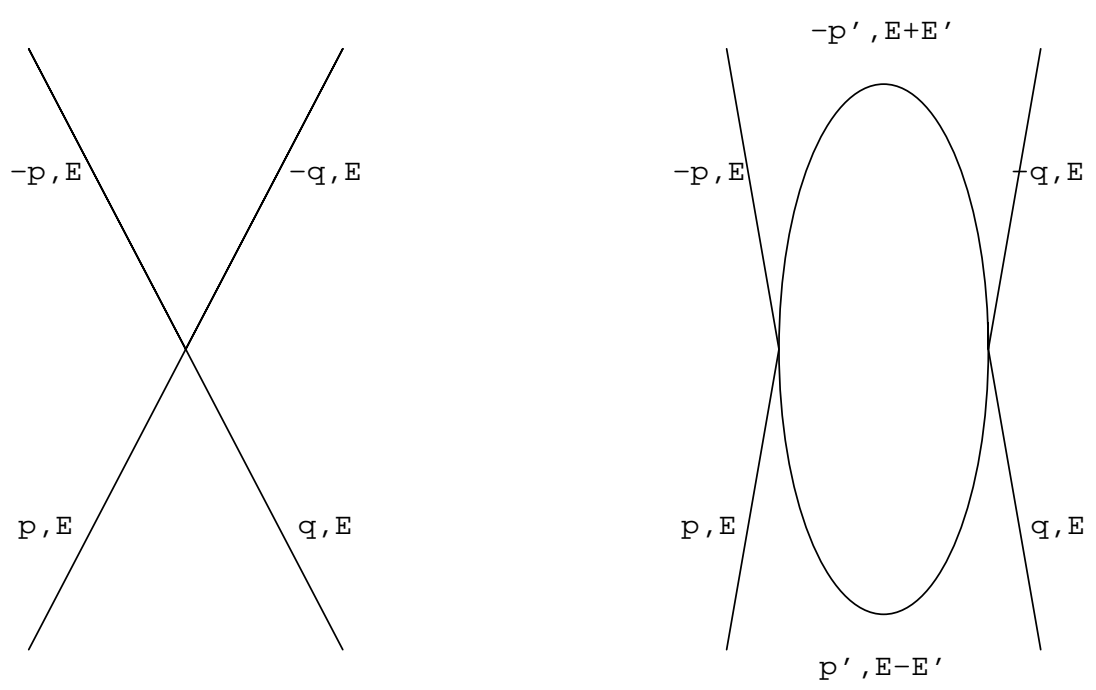

Fig.1.4 Four-fermion vertex with the marginal coupling (sum of incoming momenta $\vec{p}+(-\vec{p})=0)$, together with the loop correction; $+\vec{q}$ and $-\vec{q}$ are the final momenta.

The origin of the attractive interaction lies in the electron-phonon interactions in the metal. In order to study them we substitute them with an effective four-fermion interaction that, as we know, for special values of the momenta is marginal.

The importance of the marginal interactions depends on the quantum corrections because they can produce the effect of reducing or increasing the coupling. Let us therefore show that, if one considers the loop diagram of Fig 1.4, the attractive interaction becomes stronger.

\footnotetext{
${ }^{6}$ The BCS theory, valid in the limit of weak electron-phonon coupling, is generalized to strong coupling by the Eliashberg theory 41].
} 
To compute Fig 1.4 we use the coupling (1.23) assuming, for simplicity a constant interacting potential $V$. The fermion propagator can be easily derived from the (1.19). The energy and the longitudinal momentum integration are cut-off, so that one is actually considering only momenta near the Fermi surface.

The sum of the two diagrams is given by

$$
\begin{aligned}
& V(E)=V-V^{2} \times \\
\times & \int \frac{d E^{\prime} d^{2} \vec{k} d \ell}{(2 \pi)^{4}\left[\left(E+E^{\prime}\right)(1+i \epsilon)-v_{F}(\vec{k}) \ell\right]\left[\left(E-E^{\prime}\right)(1+i \epsilon)-v_{F}(\vec{k}) \ell\right]} \\
= & V-V^{2} N \ln \frac{\delta}{E},
\end{aligned}
$$

where

$$
N=\int \frac{d^{2} \vec{k}}{(2 \pi)^{3} v_{F}(\vec{k})}
$$

and $\delta$ is the upper cutoff in the energy integration. From (1.26) we get, at the same order of approximation,

$$
V(E) \approx \frac{V}{1+N V \ln \frac{\delta}{E}}
$$

which shows that, when $E \rightarrow 0$, if $V>0$ (repulsive interaction), $V(E)$ becomes weaker and weaker while, if $V<0$ (attractive interaction), the coupling becomes more negative and stronger. Therefore one expects that, if $V<0$, at zero temperature the naive vacuum is unstable and the formation of fermion pairs is favored.

For metals at low temperatures the situation is as follows. If at some intermediate scale $E_{1}=\left(\frac{m}{M}\right)^{1 / 2} \delta(m=$ electron mass, $M=$ nucleus mass $)$ the attractive interaction is stronger than the Coulomb interaction, then at smaller energies the ordinary vacuum, filled with gapless fermions, will become unstable and the electrons will condense; if, one other hand, this condition is not satisfied, the metal is an ordinary conductor. In a superconductor there is a non-vanishing vacuum expectation value of the electron bilinear:

$$
\left\langle\psi_{e} \psi_{e}\right\rangle \neq 0
$$

The condensate $(\overline{1.29})$ acts as an order parameter and its non-vanishing value signals the phenomenon of spontaneous symmetry breaking. As the broken symmetry is a gauge symmetry, $U(1)_{e . m}$., there are no Goldstone bosons associated to it, but the photon acquires a mass, which produces, for example, the Meissner effect. This is what happens for BCS electromagnetic superconductors. For QCD with light quarks at zero density and zero temperature this mechanism is supposed to favor the formation of a $\bar{q} q$ condensate, with breaking of the chiral symmetry and associated Nambu-Goldstone bosons (the pions). For QCD at high density an attractive coupling $(V<0)$ between a quark pair is provided by the color antitriplet channel; therefore it should ultimately dominate for $T \rightarrow 0$ and $\mu \rightarrow \infty$. 


\subsection{The true vacuum}

Once we have been convinced that the naive vacuum is unstable we should try to find the true superconducting vacuum state. To do that we have to show that the formation of a quark-quark condensate occurs because of the color interaction. The interaction term is fundamentally provided by gluon exchanges and to compute it and derive the gap equation one can follow different approaches, such as, for example, the one of Bailin and Love in [1] based on the resummation of bubble diagrams. For this method and similar approaches we refer to the reviews [14].

For pedagogical purposes it is however sufficient to consider a Nambu-Jona Lasinio [42] (NJL) interaction (4 fermion interaction) that mimics the QCD interaction. In this approximation the hamiltonian is:

$$
H=\int d^{3} x\left(\bar{\psi}\left(i \not \partial+\mu \gamma_{0}\right) \psi+F \bar{\psi} \gamma^{\mu} T^{A} \psi \bar{\psi} \gamma_{\mu} T^{A} \psi\right)
$$

Here $F$ is a form factor that is introduced to take into account the asymptotic freedom property of QCD; its Fourier transform behaves as follows: $F(p) \rightarrow 0$ for $p \rightarrow \infty$, and this property also ensures the good behavior of the integrals for $p \rightarrow \infty$.

In order to obtain the gap equation we make a mean field ansatz:

$$
\psi_{\alpha i}^{T} C \psi_{\beta j} \rightarrow\left\langle\psi_{\alpha i}^{T} C \psi_{\beta j}\right\rangle .
$$

We repeat that Greek indices $\alpha, \beta$ are color indices, Latin indices $i, j$ are flavor indices. Let us now show that one expects

$$
\left\langle\psi_{\alpha i}^{T} C \psi_{\beta j}\right\rangle=\frac{\Delta}{2} \epsilon_{\alpha \beta I} \epsilon_{i j K} \Omega_{I K} .
$$

It is useful to note that, by evaluating (1.30) in mean field approximation, i.e. substituting to a pair of fermion fields in (1.30) their mean value given by (1.32) and (1.31), the interaction term takes the form

$$
\sim \Delta \psi \psi+\text { h.c. }
$$

i.e. the fermions acquire a Majorana mass.

To prove (1.32) we note that, as we have already discussed, QCD interaction favors antisymmetry in color; the pair has zero angular momentum (in this way the entire Fermi surface is available and the effect becomes macroscopic); if it has also spin zero the fermion pair must be in an antisymmetric state of flavor to produce an antisymmetric wavefunction 1 .

Clearly the form of the matrix $\Omega_{I K}$ corresponding to the true vacuum state depends on dynamical effects. In the literature two cases have been widely discussed

\footnotetext{
${ }^{7}$ With one flavor this possibility does not exist and one remains with the sole possibility of a condensate with angular momentum equal to 1 ; in this case, however the superconducting effect is more modest, with gaps of the order of $1 \mathrm{MeV} 43$.
} 
1. Color-Flavor-Locking (CFL): $\Omega_{I K}=\delta_{I K}$, see (1.5);

2. 2 flavor SuperConductivity (2SC): $\Omega_{I K}=\delta_{I 3} \delta_{K 3}$, see (1.3),

and we will concentrate our attention on them (other cases have been also considered; we refer to the literature, as reviewed for example in [14])

The CFL model takes its name from the well known property

$$
\sum_{K=1}^{3} \epsilon_{\alpha \beta K} \epsilon_{i j K}=\delta_{\alpha i} \delta_{\beta j}-\delta_{\alpha j} \delta_{\beta i},
$$

by which the color and flavor indices become mixed and indeed locked together.

The 2SC model corresponds to the presence in the condensate of only the $u$ and $d$ flavors: this means that this ansatz may be relevant in the situation characterized by an intermediate chemical potential $m_{u}, m_{d} \ll \mu \ll m_{s}$. Therefore one can imagine of having taken the limit $m_{s} \rightarrow \infty$ and the theory is effectively a two-flavor theory.

Let us now introduce $a, a^{\dagger}, b, \ldots$ annihilation/creation operators of particles and holes:

$$
\psi_{\alpha i}=\sum_{k} u_{k}\left(a_{k} e^{i k x}+b_{k}^{\dagger} e^{-i k x}\right)_{\alpha i} .
$$

The existence of the condensate gives rise to the following hamiltonian

$$
\begin{aligned}
H & =\sum_{\vec{k}}\left(|k-\mu| a_{k}^{\dagger} a_{k}+(k+\mu) b_{k}^{\dagger} b_{k}\right) \\
& +\sum_{\vec{k}} \frac{\Delta F^{2}}{2} e^{-i \Phi} a_{k} a_{-k}+\frac{\Delta F^{2}}{2} e^{+i \Phi} b_{k}^{\dagger} b_{-k}^{\dagger}+h c
\end{aligned}
$$

where we have omitted for simplicity any color-flavor indices as we wish to stress the mechanism that produces the condensate. Let us now perform a unitary Bogolubov-Valatin [44] transformation. To do that we introduce annihilation and creation operators for quasiparticle and quasiholes

$$
\begin{aligned}
& y_{k}=\cos \theta a_{k}-e^{i \Phi} \sin \theta a_{-k}^{\dagger}, \\
& z_{k}=\cos \varphi b_{k}-e^{i \Phi} \sin \varphi b_{-k}^{\dagger} .
\end{aligned}
$$

By an appropriate choice of the parameters of the transformation we can transform the original hamiltonian in a new hamiltonian describing a gas of non interacting quasiparticles

$$
H=\sum\left[\epsilon_{y}(k) y_{k}^{\dagger} y_{k}+\epsilon_{z}(k) z_{k}^{\dagger} z_{k}\right]
$$

with

$$
\begin{aligned}
& \epsilon_{y}(k)=\sqrt{(k-\mu)^{2}+\Delta^{2} F^{4}}, \\
& \epsilon_{z}(k)=\sqrt{(k-\mu)^{2}+\Delta^{2} F^{4}} .
\end{aligned}
$$

These equations show two effects: 
1. The quasiparticles and quasiholes are free, there is no interaction term;

2. in the dispersion laws of the quasiparticles a mass term proportional to $\Delta$, called gap parameter, appears.

To obtain (1.37), i.e. a free hamiltonian, the parameters have to be chosen as follows:

$$
\begin{aligned}
\cos 2 \theta & =\frac{|k-\mu|}{\sqrt{|k-\mu|^{2}+\Delta^{2} F^{4}}}, \\
\cos 2 \varphi & =\frac{k+\mu}{\sqrt{\mid(k+\mu)^{2}+\Delta^{2} F^{4}}}
\end{aligned}
$$

We note that, differently from the original $a_{k}, b_{k}$ operators that annihilate the false vacuum, the quasiparticles annihilation operators destroy the true vacuum

$$
y_{k}\left|0>=0, \quad z_{k}\right| 0>=0 .
$$

We have still to prove that $\Delta \neq 0$. We do that and get an equation for $\Delta$ by substituting for $y_{k}, z_{k}$ in eq. (1.32). We get in this way an integral equation that has the following schematic form (gap equation):

$$
\Delta=C \int d k F^{2}(k)\left[\frac{\Delta}{\sqrt{(k-\mu)^{2}+\Delta^{2} F^{4}}}+\frac{\Delta}{\sqrt{(k+\mu)^{2}+\Delta^{2} F^{4}}}\right] .
$$

We see immediately that the origin of the instability of the false vacuum lies in the first of the two terms in the r.h.s. of (1.41): If $\Delta=0$ there is no compensation for the divergence at $k=\mu$.

To be more quantitative we have to consider the two models in more detail. We differ this quantitative analysis to Section 2 , while giving here only the results.

CFL model $\left(m_{s}=m_{u}=m_{d}=0\right)$.

In this case all the $3 \times 3=9$ quarks acquire a Majorana mass. The CFL condition gives two different set of eigenvalues. The first one comprises 8 degenerate masses $\Delta_{1}=\Delta_{2}=\ldots=\Delta_{8}$ and the second set the non degenerate mass $\Delta_{9}$. The actual values of the gaps depends on the model and the approximations involved; typical values are

$$
\begin{aligned}
& \Delta_{1}=\ldots=\Delta_{8}=\Delta \approx 80 \mathrm{MeV}, \\
& \Delta_{9} \approx-2 \Delta,
\end{aligned}
$$

for $\mu \approx 400 \mathrm{MeV}$ (a numerical analysis for this case is contained in Section 2.6). The interaction couples quarks of different flavor and color. Moreover, since we start with a massless theory, the pairing occurs for left-handed and right handed quarks separately. Therefore these condensates break $S U(3)_{c} \otimes$ $S U(3)_{L} \otimes S U(3)_{R}$; finally, since the pair has baryonic number $2 / 3$, also $U(1)_{B}$ is 
broken. It should be observed, however, that a diagonal $S U(3)_{c+L+R}$ symmetry remains unbroken.

2SC model $\left(m_{s} \gg \mu \gg m_{u}, m_{d}\right)$.

In this case there are 4 massive quarks (the up and down quarks with colors 1 and 2), while the strange quark with any color and the $u$ and $d$ quarks with color 3 remain ungapped. Numerical results for this case are qualitatively in agreement with the results (1.42) of the CFL model (see for example Section 2.6).

One can show, using the Schwinger-Dyson equation (also called in this context the Nambu-Gorkov equation), that, for arbitrary large $\mu$, the CFL model is favored [6]; however, for intermediate $\mu$ the $2 \mathrm{SC}$ state can be more stable and the phase diagram should resemble that depicted in Fig. 1.1.

The spontaneous breaking of global symmetries implies the existence of Nambu Goldstone bosons; for internal symmetries there are as many NGBs as there are broken generators $G_{a}$. The NGBs are massless scalar particles that interpolate among different degenerate vacua

$$
\left[H, G^{a}\right]=0 \quad G^{a} \mid 0>\neq 0 .
$$

As they are massless, they are the lowest energy quasiparticles of the effective theory. On the other hand, for broken local gauge symmetries, by the HiggsAnderson mechanism the gauge bosons acquire masses and there are no NGBs. In CFL model the diquark condensate (1.5) breaks $S U(3)_{L} \times S U(3)_{R} \times S U(3)_{c} \times$ $U(1)_{B}$ to $S U(3)_{c+L+R} \times Z_{2}$. All the 9 quarks are massive and they belong to a $S U(3)_{c+L+R}$ singlet and a $S U(3)_{c+L+R}$ octet. All the 8 gluons are massive and are degenerate. There are $8+1 \mathrm{Nambu-Goldstone}$ bosons. In the 2SC model the condensate (1.3) breaks $S U(3)_{c} \otimes S U(2)_{L} \otimes S U(2)_{R} \times U(1)_{B}$ down to $S U(2)_{c} \times S U(2)_{L} \times S U(2)_{R}$. While the chiral group is unbroken, $S U(3)_{c}$ is broken to $S U(2)_{c}$. Therefore 3 gluons remain massless and 5 acquire a mass. As to the other quasiparticles, there is one would-be NGB associated to the breaking of the axial color; moreover of the 6 quarks (2 flavors in 3 colors) 4 are massive and 2 are massless.

\subsection{Effective lagrangian for the NGB in the CFL model}

We wish now derive an effective lagrangian for the low energy excitations (NGBs) of the CFL model [20]. In order to do that let us introduce two coset fields $X$ and $Y$ transforming under the symmetry group as the left handed and right handed quark fields

$$
\begin{gathered}
X \rightarrow g_{c} X U_{L}^{T}, \\
Y \rightarrow g_{c} Y U_{R}^{T} .
\end{gathered}
$$


$X$ and $Y$ contain both 8 scalar fields

$$
\begin{aligned}
& X=\exp \left\{i \frac{\tilde{\Pi}_{X}^{A} T^{A}}{F}\right\} \\
& Y=\exp \left\{i \frac{\tilde{\Pi}_{Y}^{A} T^{A}}{F}\right\}
\end{aligned}
$$

corresponding to the $16 \mathrm{NGBs}$ induced by the spontaneous symmetry breaking $S U(3)_{c} \times S U(3)_{L} \times S U(3)_{R} \rightarrow S U(3)_{c+L+R}$ (we neglect for the moment the $U(1)_{B}$ factor $)$. We introduce the gauge covariant currents

$$
\begin{aligned}
& J_{X}^{\mu}=X \partial^{\mu} X^{\dagger}+g^{\mu}, \\
& J_{Y}^{\mu}=Y \partial^{\mu} Y^{\dagger}+g^{\mu},
\end{aligned}
$$

where

$$
g_{\mu}=i g T^{A} A_{\mu}^{A}
$$

is the gluon field. Under the gauge group these currents transform as follows:

$$
\begin{aligned}
& J_{X}^{\mu} \rightarrow g_{c} J_{X}^{\mu} g_{c}^{\dagger} \\
& J_{Y}^{\mu} \rightarrow g_{c} J_{Y}^{\mu} g_{c}^{\dagger} .
\end{aligned}
$$

The most general gauge invariant (but not Lorentz invariant) lagrangian containing $X, Y$ and $g_{\mu}$ fields is as follows

$$
\begin{aligned}
\mathcal{L}_{e f f} & =-\frac{F^{2}}{4} \tilde{g}_{\mu \nu}\left\{\operatorname{Tr}\left[\left(J_{X}^{\mu}-J_{Y}^{\mu}\right)\left(J_{X}^{\nu}-J_{Y}^{\nu}\right)\right]\right. \\
& \left.+\alpha \operatorname{Tr}\left[\left(J_{X}^{\mu}+J_{Y}^{\mu}\right)\left(J_{X}^{\nu}+J_{Y}^{\nu}\right)\right]\right\} \\
& + \text { (kinetic term for } g),
\end{aligned}
$$

where $\tilde{g}_{\mu \nu}=\operatorname{diag}(1,-v,-v,-v)$ and $F, \alpha$ are parameters. We will prove in par. 2.3.2 that an effective lagrangian of this type for the scalar fields is generated by loop corrections. We rewrite this equation as follows

$$
\begin{aligned}
\mathcal{L}_{e f f} & =-\frac{F^{2}}{4} \tilde{g}_{\mu \nu}\left\{\operatorname{Tr}\left[\left(X \partial_{\mu} X^{\dagger}-Y \partial_{\mu} Y^{\dagger}\right)\left(X \partial_{\nu} X^{\dagger}-Y \partial_{\nu} Y^{\dagger}\right)\right]\right. \\
& \left.+\alpha \operatorname{Tr}\left[\left(X \partial_{\mu} X^{\dagger}+Y \partial_{\mu} Y^{\dagger}+2 g_{\mu}\right)\left(X \partial_{\nu} X^{\dagger}+Y \partial_{\nu} Y^{\dagger}+2 g_{\nu}\right)\right]\right\} \\
& + \text { (kinetic term for } g) .
\end{aligned}
$$

Note that in this equation one has both a Debye and a Meissner mass for the gluon, $m_{D}^{2}=\alpha g^{2} F^{2}, m_{M}^{2}=v^{2} \alpha m_{D}^{2}$ corresponding to a mass term for $A_{0}$ and $\vec{A}$, respectively; they can be read from $(1.50)$. We will show in par. 2.3 .2 that one has to put $\alpha=1$ in order to have the correct normalization for the gluon masses. Since $F=\mathcal{O}(\mu)$, in the limit of small momenta, one can neglect the kinetic gluon term and, because of the equations of motion, the gluon field becomes an auxiliary field given by

$$
g_{\mu}=-\frac{1}{2}\left(X \partial_{\mu} X^{\dagger}-Y \partial_{\mu} Y^{\dagger}\right) .
$$


Therefore the lagrangian (1.50) reduces to

$$
\mathcal{L}_{e f f}=-\frac{F^{2}}{4} \tilde{g}_{\mu \nu} \operatorname{Tr}\left[\left(X \partial_{\mu} X^{\dagger}-Y \partial_{\mu} Y^{\dagger}\right)\left(X \partial_{\nu} X^{\dagger}-Y \partial_{\nu} Y^{\dagger}\right)\right] .
$$

If we put

$$
\Sigma=Y^{\dagger} X=\exp \left\{\frac{2 i \Pi^{A} T^{A}}{F}\right\}
$$

$T^{A}=\lambda^{A} / 2$, from $(1.52)$ we finally obtain

$$
\mathcal{L}_{e f f}=\frac{F^{2}}{4} \operatorname{Tr}\left(\partial_{t} \Sigma \partial_{t} \Sigma^{\dagger}-v^{2} \vec{\nabla} \Sigma \cdot \vec{\nabla} \Sigma^{\dagger}\right) .
$$

We note that the matrix $\Sigma$ transforms under the chiral group as

$$
\Sigma \rightarrow U_{L} \Sigma U_{R}^{\dagger}
$$

The values of the parameters in (1.52) have been derived by several authors (the derivation in the framework of the effective theory will be presented in Section 2). One finds [45, 46, 47, 48, 49, 50] :

$$
F^{2}=\frac{\mu^{2}(21-8 \ln 2)}{36 \pi^{2}}, \quad v^{2}=\frac{1}{3} .
$$

A similar analysis can be done for the superfluid mode [20], 45]; we can therefore write down the low energy effective lagrangian including both the $S U(3)$ and the $U(1)$ NGBs as follows:

$$
\begin{aligned}
\mathcal{L}_{N G B} & =\frac{F^{2}}{4} \operatorname{Tr}\left(\partial_{t} \Sigma \partial_{t} \Sigma^{\dagger}-\frac{1}{3} \vec{\nabla} \Sigma \cdot \vec{\nabla} \Sigma^{\dagger}\right) \\
& +\frac{f_{\sigma}^{2}}{2}\left(\partial_{t} U \partial_{t} U^{\dagger}-\frac{1}{3} \vec{\nabla} U \cdot \vec{\nabla} U^{\dagger}\right)
\end{aligned}
$$

where

$$
U=\exp \left\{\frac{i \sigma}{f_{\sigma}}\right\}, \quad f_{\sigma}^{2}=9 \mu^{2} / \pi^{2},
$$

see below, eq. (2.75).

The lagrangian (1.49) is completely analogous to the hidden gauge symmetry version of the chiral lagrangian of QCD [51], but for the breaking of the Lorentz invariance: $g_{\mu \nu} \neq \tilde{g}_{\mu \nu}$, due to the high density.

We finally note that there is an extra $U(1)_{A}$ symmetry in the lagrangian, which is broken by instantons. Even though the contribution of the instantons is parametrically small at asymptotic densities, it is sufficient to forbid the presence of an extra massless scalar 8 . Notice that one has no NGB associated to the breaking of the chiral symmetry, because, as the non-vanishing condensates only couple quarks with the same helicities, chiral symmetry is broken only indirectly, by the fact that the mechanism of Color Flavor Locking locks left handed quarks to color as well as right handed quarks to color.

\footnotetext{
${ }^{8}$ Nevertheless, because of this suppression, this extra pseudoscalar is expected to be rather light. It can be observed that the instanton interaction is suppressed not only by the asymptotic energies, but also by the fact that, being a 6-quark coupling, it becomes irrelevant at the Fermi surface.
} 


\section{Chapter 2}

\section{High Density Effective Theory}

\subsection{The 2-D effective theory}

We wish now to derive an effective description of QCD at very high baryonic chemical potential $\mu$ and very small temperature $(T \rightarrow 0)$.

To begin with we consider the equations of motion for a massless quark at finite density

$$
\left(i \not D+\mu \gamma_{0}\right) \psi=0
$$

where $\mu=$ is the quark number chemical potential. The $\mu \gamma_{0}$ term corresponds to a $\mu N=\mu \psi^{\dagger} \psi$ term in the hamiltonian. We note explicitly the breaking of Lorentz invariance of this equation, which, however, is still symmetric under rotations. At $T=0$ the vacuum is characterized by the fact that the fermions fill in all the low energy states up to a maximum energy, the Fermi energy. Due to the Pauli principle, the interaction of low energy quarks implies the exchange of high momenta, which is disfavored by the Asymptotic Freedom property of QCD. Therefore only the fermions that are near the Fermi surface can interact. Since they have high momentum $(p \simeq \mu)$ they are almost free and we can write:

$$
D^{\mu} \rightarrow \partial^{\mu}=-i p^{\mu}
$$

i.e. in momentum space:

$$
\left(\not p+\mu \gamma_{0}\right) \psi=0
$$

or $\left(\vec{\alpha}=\gamma_{0} \vec{\gamma}\right)$ :

$$
p_{0} \psi=(\vec{\alpha} \cdot \vec{p}-\mu) \psi .
$$

Let us now consider the eigenvalue equation for the Dirac hamiltonian:

$$
H_{D}=p_{0}=\vec{\alpha} \cdot \vec{p}-\mu,
$$

whose eigenvalues can be found solving the equation $\left(p_{0}+\mu\right)^{2}=\vec{p}^{2}$. There are positive and negative energies, given by

$$
p_{0}=E_{ \pm} \equiv-\mu \pm|\vec{p}|
$$


with eigenvectors

$$
\psi_{ \pm}=P_{ \pm} \psi \equiv \frac{1}{2}\left(1 \pm \frac{\vec{\alpha} \cdot \vec{p}}{|\vec{p}|}\right) \psi
$$

Therefore at high-density the energy spectrum of a massless fermion is described by states $| \pm\rangle$ with energies given by (2.3). As we have already stressed, for energies much lower than the Fermi energy $\mu$, among the states $|+\rangle$ only those close to the Fermi surface. i.e. with $|\vec{p}| \approx \mu$, can be excited. As to the states $|-\rangle$, they have $E_{-} \approx-2 \mu$ and therefore decouple; this means that in the physical spectrum only the positive energy states $|+\rangle$ and the gluons remain.

After this quantum mechanical introduction, let us consider the field theoretical version of the previous argument. The main idea of the effective theory is the observation that the quarks participating in the dynamics have large $(\sim \mu)$ momenta. Wherefore one can introduce velocity dependent fields by extracting the large part $\mu \vec{v}$ of this momentum. One starts with the Fourier decomposition of the quark field $\psi(x)$ :

$$
\psi(x)=\int \frac{d^{4} p}{(2 \pi)^{4}} e^{-i p \cdot x} \psi(p),
$$

and introduces the quark velocity by

$$
p^{\mu}=\mu v^{\mu}+\ell^{\mu},
$$

where

$$
v^{\mu}=(0, \vec{v})
$$

with $|\vec{v}|=1$. Let us put

$$
\ell^{\mu}=\left(\ell^{0}, \vec{\ell}\right)
$$

and

$$
\vec{\ell}=\vec{v} \ell_{\|}+\vec{\ell}_{\perp}
$$

with

$$
\vec{\ell}_{\perp}=\vec{\ell}-(\vec{\ell} \cdot \vec{v}) \vec{v}
$$

We can always choose the velocity parallel to $\vec{p}$, so that $\vec{\ell}_{\perp}=0$ and

$$
\int d^{4} p=\mu^{2} \int d \Omega \int d \ell_{\|} \int_{-\infty}^{+\infty} d \ell_{0}=4 \pi \mu^{2} \int \frac{d \vec{v}}{4 \pi} \int d \ell_{\|} \int_{-\infty}^{+\infty} d \ell_{0} .
$$

In general we will be interested only in the degrees of freedom at the Fermi surface therefore we can limit the integration limits as follows:

$$
-\delta \leq \ell_{\|} \leq+\delta
$$

with $\delta \leq \mu$ and $\delta$ much larger than any other energy scale, in particular the energy gap masses, if any. By this hypothesis

$$
\int d^{3} p=4 \pi \mu^{2} \sum_{\vec{v}}^{\prime} \int_{-\delta}^{+\delta} d \ell_{\|},
$$

\footnotetext{
${ }^{1}$ There are some important cases where this limitation does not apply, see below.
} 
with

$$
\sum_{\vec{v}}^{\prime} \equiv \int \frac{d \vec{v}}{4 \pi}
$$

eventually we take the limit $\delta \rightarrow+\infty$. In this way the Fourier decomposition (2.5) takes the form

$$
\psi(x)=\sum_{\vec{v}}{ }^{\prime} e^{-i \mu v \cdot x} \int \frac{d^{4} \ell}{(2 \pi)^{4}} e^{-i \ell \cdot x} \psi_{\vec{v}}(\ell),
$$

where

$$
\int \frac{d^{4} \ell}{(2 \pi)^{4}}=\frac{4 \pi \mu^{2}}{(2 \pi)^{4}} \int d^{2} \ell
$$

while $\psi_{\vec{v}}(\ell)$ are velocity-dependent fields. The projection operators in (2.4) can be approximated as follows:

$$
P_{ \pm}=\frac{1 \pm \vec{\alpha} \cdot \vec{v}}{2}
$$

and one can write

$$
\psi(x)=\sum_{\vec{v}} e^{-i \mu v \cdot x}\left[\psi_{+}(x)+\psi_{-}(x)\right],
$$

where

$$
\psi_{ \pm}(x)=P_{ \pm} \psi_{\vec{v}}(x)=P_{ \pm} \int \frac{d^{4} \ell}{(2 \pi)^{4}} e^{-i \ell \cdot x} \psi_{\vec{v}}(\ell)
$$

It is clear that $\psi_{ \pm}$are velocity dependent fields corresponding to positive and energy solutions of the Dirac equation.

Let us now define

$$
\begin{array}{rlrl}
V^{\mu} & =(1, \vec{v}), & & \tilde{V}^{\mu}=(1,-\vec{v}), \\
\gamma_{\|}^{\mu}=\left(\gamma^{0},(\vec{v} \cdot \vec{\gamma}) \vec{v}\right), & \gamma_{\perp}^{\mu}=\gamma^{\mu}-\gamma_{\|}^{\mu} .
\end{array}
$$

Moreover we use

$$
\begin{aligned}
& \bar{\psi}_{+} \gamma^{\mu} \psi_{+}=V^{\mu} \bar{\psi}_{+} \gamma^{0} \psi_{+}, \\
& \bar{\psi}_{-} \gamma^{\mu} \psi_{-}=V^{\mu} \bar{\psi}_{-} \gamma^{0} \psi_{-}, \\
& \bar{\psi}_{+} \gamma^{\mu} \psi_{-}=V^{\mu} \bar{\psi}_{+} \gamma_{\perp}^{\mu} \psi_{-}, \\
& \bar{\psi}_{-} \gamma^{\mu} \psi_{+}=V^{\mu} \bar{\psi}_{-} \gamma_{\perp}^{\mu} \psi_{+} .
\end{aligned}
$$

Substituting into the Dirac part of the QCD lagrangian we obtain, in the hypothesis that the residual momenta $\ell$ are small,

$$
\begin{aligned}
\mathcal{L}_{D} & =\sum_{\vec{v}}{ }^{\prime}\left[\psi_{+}^{\dagger} i V \cdot D \psi_{+}+\psi_{-}^{\dagger}(2 \mu+i \tilde{V} \cdot D) \psi_{-}+\right. \\
& \left.+\left(\bar{\psi}_{+} i D D_{\perp} \psi_{-}+\text {h.c. }\right)\right]
\end{aligned}
$$

$D_{\perp}=D_{\mu} \gamma_{\perp}^{\mu}$ and $D_{\mu}$ is the covariant derivative: $D^{\mu}=\partial^{\mu}+i g A^{\mu}$. We note that here quark fields are evaluated at the same Fermi velocity; off-diagonal terms 
are subleading due to the Riemann-Lebesgue lemma, as they are cancelled by the rapid oscillations of the exponential factor in the $\mu \rightarrow \infty$ limit. One may call this phenomenon Fermi velocity superselection rule, in analogy with the behaviour of QCD in the $m_{Q} \rightarrow \infty$ limit, where the corresponding effective theory, the Heavy Quark Effective Theory exhibits a similar phenomenon 30. By the same analogy we may refer to the present effective theory as High Density Effective Theory (HDET),

We can get rid of the negative energy solutions by integrating out the $\psi_{-}$ fields in the generating functional; at the tree level this corresponds to solve the equations of motion, which gives

$$
i V \cdot D \psi_{+}=0
$$

and

$$
\psi_{-}=-\frac{i}{2 \mu+i \tilde{V} \cdot D} \gamma_{0} \not D_{\perp} \psi_{+} .
$$

This equation shows the decoupling of $\psi_{-}$in the $\mu \rightarrow \infty$ limit. Therefore, in this limit, the $\psi_{-}$field plays no role. We also note that in the equation for $\psi_{+}$ only the energy and the momentum parallel to the Fermi velocity are relevant and the effective theory is two-dimensional.

We can write the effective lagrangian at the next to leading order in $\mu$ as follows:

$$
\mathcal{L}=-\frac{1}{4} F_{\mu \nu}^{a} F^{a \mu \nu}+\mathcal{L}_{D}
$$

where

$$
\mathcal{L}_{D}=\sum_{\vec{v}}^{\prime}\left[\psi^{\dagger} i V \cdot D \psi-\psi^{\dagger} \frac{1}{2 \mu+i \tilde{V} \cdot D} D_{\perp}^{2} \psi\right]
$$

Using the identity

$$
\psi_{+}^{\dagger} \gamma_{\perp}^{\mu} \gamma_{\perp}^{\nu} \psi_{+}=\psi_{+}^{\dagger} P^{\mu \nu} \psi_{+}
$$

where

$$
P^{\mu \nu}=g^{\mu \nu}-\frac{1}{2}\left[V^{\mu} \tilde{V}^{\nu}+V^{\nu} \tilde{V}^{\mu}\right],
$$

we can write (2.18) as follows

$$
\mathcal{L}_{D}=\sum_{\vec{v}}^{\prime}\left[\psi^{\dagger} i V \cdot D \psi-P^{\mu \nu} \psi^{\dagger} \frac{1}{2 \mu+i \tilde{V} \cdot D} D_{\mu} D_{\nu} \psi\right] .
$$

We can make this equation more symmetric by introducing positive energy fields with opposite velocity:

$$
\psi_{ \pm} \equiv \psi_{+, \pm \vec{v}} .
$$

Even though we use the same notation $\psi_{ \pm}$here and for the positive/negative energy fields, we stress that $\psi_{ \pm}$in (2.21) and in the sequel are positive energy 
fields, with opposite Fermi velocities: $\pm \vec{v}$. Eq. (2.20) can be therefore written as follows:

$$
\begin{aligned}
\mathcal{L}_{D} & =\sum_{\vec{v}}\left[\psi_{+}^{\dagger} i V \cdot D \psi_{+}+\psi_{-}^{\dagger} i \tilde{V} \cdot D \psi_{-}\right. \\
& \left.-\psi_{+}^{\dagger} \frac{P^{\mu \nu}}{2 \mu+i \tilde{V} \cdot D} D_{\mu} D_{\nu} \psi_{+}-\psi_{-}^{\dagger} \frac{P^{\mu \nu}}{2 \mu+i V \cdot D} D_{\mu} D_{\nu} \psi_{-}\right] .
\end{aligned}
$$

To this lagrangian involving only the left handed fields, one should add a similar one containing the right handed fermionic fields. We observe that a factor $\frac{1}{2}$ has been introduced in the definition of the sum over the Fermi velocities that now reads:

$$
\sum_{\vec{v}}=\int \frac{d \vec{v}}{8 \pi} .
$$

This extra factor is needed because, after the introduction of the fields with opposite $\vec{v}, \psi_{-}=\psi_{+,-\vec{v}}$, we have doubled the degrees of freedom and therefore we must integrate only over half solid angle. Apart from this factor there should be another 7 factor $\frac{1}{2}$ in (2.22), but we got rid of it by a redefinition of the fermion field,

$$
\psi \rightarrow \frac{1}{\sqrt{2}} \psi
$$

We close this section by a definition that will be useful in the sequel:

$$
\mathcal{L}_{D}=\mathcal{L}_{0}+\mathcal{L}_{1}+\mathcal{L}_{2}
$$

with

$$
\begin{aligned}
& \mathcal{L}_{0}=\sum_{\vec{v}}\left[\psi_{+}^{\dagger} i V \cdot \partial \psi_{+}+\psi_{-}^{\dagger} i \tilde{V} \cdot \partial \psi_{-}\right], \\
& \mathcal{L}_{1}=i g \sum_{\vec{v}}\left[\psi_{+}^{\dagger} i V \cdot A \psi_{+}+\psi_{-}^{\dagger} i \tilde{V} \cdot A \psi_{-}\right],
\end{aligned}
$$

and

$$
\mathcal{L}_{2}=-\sum_{\vec{v}} P^{\mu \nu}\left[\psi_{+}^{\dagger} \frac{1}{2 \mu+i \tilde{V} \cdot D} D_{\mu} D_{\nu} \psi_{+}+\psi_{-}^{\dagger} \frac{1}{2 \mu+i V \cdot D} D_{\mu} D_{\nu} \psi_{-}\right]
$$

$\mathcal{L}_{0}$ is the free quark lagrangian (without gap mass terms); $\mathcal{L}_{1}$ represents the coupling of quarks to one gluon; $\mathcal{L}_{2}$ is a non local lagrangian containing couplings of two quarks to any number of gluons. Its effect will be discussed in section 2.4.1.

\subsection{HDET for superconducting phases}

The construction described above is valid for any theory describing massless fermions at high density provided one excludes degrees of freedom very far from

\footnotetext{
${ }^{2}$ In [29] this factor explicitly appears in the lagrangian; here we have simplified the notation.
} 
the Fermi surface. As discussed in Section 1, however, for small temperature and high density the fermions are likely to be gapped due to the phenomenon of the color superconductivity, We shall examine the modification in the formalism in two models, i.e. the Color Flavor Locking, CFL, model (3 massless quarks: $\left.m_{u}, m_{d}, m_{s} \ll \mu\right)$ and the Two-Flavor, 2SC, model $\left(m_{s} \rightarrow \infty\right)$

\subsubsection{HDET in the CFL phase}

In the Color Flavor Locking phase the symmetry breaking is induced by the condensates

$$
\left\langle\psi_{\alpha i}^{L T} C \psi_{\beta j}^{L}\right\rangle=-\left\langle\psi_{\alpha i}^{R T} C \psi_{\beta j}^{R}\right\rangle=\frac{\Delta}{2} \epsilon_{\alpha \beta I} \epsilon_{i j I},
$$

where $\psi^{L, R}$ are Weyl fermions and $C=i \sigma_{2}$. Eq. (2.28) corresponds to the invariant coupling $\left(\psi \equiv \psi_{L}\right)$ :

$$
-\frac{\Delta}{2} \sum_{I=1,3} \psi^{T} C \epsilon_{I} \psi \epsilon_{I}-(L \rightarrow R)+h . c .,
$$

and

$$
\left(\epsilon_{I}\right)_{a b}=\epsilon_{I a b} .
$$

For the Dirac fermions $\psi_{ \pm}$we introduce the compact notation

$$
\chi=\left(\begin{array}{c}
\psi_{+} \\
C \psi_{-}^{*}
\end{array}\right)
$$

and we use a different basis for quark fields:

$$
\psi_{ \pm}=\frac{1}{\sqrt{2}} \sum_{A=1}^{9} \lambda_{A} \psi_{ \pm}^{A} .
$$

The CFL fermionic lagrangian has therefore the form:

$$
\begin{aligned}
\mathcal{L}_{D} & =\mathcal{L}_{0}+\mathcal{L}_{1}+\mathcal{L}_{\Delta}= \\
& =\sum_{\vec{v}} \sum_{A, B=1} \chi^{A \dagger}\left(\begin{array}{cc}
i \operatorname{Tr}\left[T_{A} V \cdot D T_{B}\right] & \Delta_{A B} \\
\Delta_{A B} & i \operatorname{Tr}\left[T_{A} \tilde{V} \cdot D^{*} T_{B}\right]
\end{array}\right) \chi^{B} \\
& +(L \rightarrow R)
\end{aligned}
$$

where

$$
\Delta_{A B}=\Delta \operatorname{Tr}\left[\epsilon_{I} T_{A}^{T} \epsilon_{I} T_{B}\right]
$$

and

$$
T_{A}=\frac{\lambda_{A}}{\sqrt{2}}
$$

Here $\lambda_{9}=\lambda_{0}=\sqrt{\frac{2}{3}} \times \mathbf{1}$. We use the identity $(g$ any $3 \times 3$ matrix $)$ :

$$
\epsilon_{I} g^{T} \epsilon_{I}=g-\operatorname{Tr} g
$$


we obtain

$$
\Delta_{A B}=\Delta_{A} \delta_{A B}
$$

where

$$
\Delta_{1}=\cdots=\Delta_{8}=\Delta
$$

and

$$
\Delta_{9}=-2 \Delta
$$

The CFL free fermionic lagrangian assumes therefore the form:

$$
\mathcal{L}_{0}+\mathcal{L}_{\Delta}=\sum_{\vec{v}} \sum_{A=1}^{9} \chi^{A \dagger}\left(\begin{array}{cc}
i V \cdot \partial & \Delta_{A} \\
\Delta_{A} & i \tilde{V} \cdot \partial
\end{array}\right) \chi^{A}+(L \rightarrow R) .
$$

Here the average over the velocities is given by (2.23). From this equation one can immediately obtain the free fermion propagator that in momentum space is given by:

$$
S_{A B}(p)=\frac{\delta_{A B}}{V \cdot p \tilde{V} \cdot p-\Delta_{A}^{2}+i \epsilon}\left(\begin{array}{cc}
\tilde{V} \cdot p & -\Delta_{A} \\
-\Delta_{A} & V \cdot p
\end{array}\right)
$$

We note that this propagator is analogous to the Nambu-Gorkov propagator of the full theory.

\subsubsection{HDET in the 2SC phase}

For the two flavour case, which encompasses both the 2SC model and the existing calculation in the LOFF phase we follow a similar approach.

The symmetry breaking is induced by the condensates

$$
\left\langle\psi_{\alpha i}^{L T} C \psi_{\beta j}^{L}\right\rangle=-\left\langle\psi_{\alpha i}^{R T} C \psi_{\beta j}^{R}\right\rangle=\frac{\Delta}{2} \epsilon_{\alpha \beta 3} \epsilon_{i j 3},
$$

corresponding to the invariant coupling $\left(\psi \equiv \psi^{L}\right)$ :

$$
\mathcal{L}_{\Delta}=-\frac{\Delta}{2} \psi^{T} C \epsilon \psi \epsilon-(L \rightarrow R)+\text { h.c. }
$$

where

$$
\epsilon=i \sigma_{2} \text {. }
$$

As in the previous section we use a different basis for the fermion fields by writing

$$
\begin{aligned}
\psi_{+, \alpha i} & =\sum_{A=0}^{3} \frac{\left(\sigma_{A}\right)_{\alpha i}}{\sqrt{2}} \psi_{+}^{A} \quad(i, \alpha=1,2) \\
\psi_{+, 31} & =\psi_{+}^{4} \\
\psi_{+, 32} & =\psi_{+}^{5}
\end{aligned}
$$

where $\sigma_{A}$ are the Pauli matrices for $A=1,2,3$ and $\sigma_{0}=1$. As usual $\psi_{+}^{A}$ are positive energy, velocity dependent fields and we also introduce $\psi_{-}^{A}$ according to the formula:

$$
\psi_{ \pm}^{A} \equiv \psi_{+, \pm \vec{v}}^{A}
$$


A different, but also useful notation for the fields $\psi_{+, \alpha i}$ uses a combination of $\lambda$ matrices, as follows

$$
\psi_{+, \alpha i}=\sum_{A=0}^{5} \frac{\left(\tilde{\lambda}_{A}\right)_{\alpha i}}{\sqrt{2}} \psi_{+}^{A}
$$

The $\tilde{\lambda}_{A}$ matrices are defined in terms of the usual $\lambda$ matrices as follows: $\tilde{\lambda}_{0}=$ $\frac{1}{\sqrt{3}} \lambda_{8}+\sqrt{\frac{2}{3}} \lambda_{0} ; \tilde{\lambda}_{A}=\lambda_{A}(A=1,2,3) ; \tilde{\lambda}_{4}=\frac{\lambda_{4-i 5}}{\sqrt{2}} ; \tilde{\lambda}_{5}=\frac{\lambda_{6-i 7}}{\sqrt{2}}$.

After the introduction, analogously to (2.30), of the fields $\chi^{A}$, the $2 \mathrm{SC}$ fermionic lagrangian assumes the form:

$$
\begin{aligned}
\mathcal{L}_{D} & =\mathcal{L}_{0}+\mathcal{L}_{1}+\mathcal{L}_{\Delta}= \\
& =\sum_{\vec{v}} \sum_{A, B=0}^{5} \chi^{A \dagger}\left(\begin{array}{cc}
i \operatorname{Tr}\left[\tilde{T}_{A} V \cdot D \tilde{T}_{B}\right] & \Delta_{A B} \\
\Delta_{A B} & i \operatorname{Tr}\left[\tilde{T}_{A} \tilde{V} \cdot D^{*} \tilde{T}_{B}\right]
\end{array}\right) \chi^{B} \\
& +(L \rightarrow R) .
\end{aligned}
$$

Here

$$
\begin{array}{ll}
\Delta_{A B}=\frac{\Delta}{2} \operatorname{Tr}\left[\epsilon \sigma_{A}^{T} \epsilon \sigma_{B}\right] & (A, B=0, \ldots 3) \\
\Delta_{A B}=0 & (A, B=4,5) .
\end{array}
$$

and

$$
\tilde{T}_{A}=\frac{\tilde{\lambda}_{A}}{\sqrt{2}} \quad(A=0, \ldots, 5) .
$$

Analogously to (2.35) we use the identity ( $g$ any $2 \times 2$ matrix):

$$
\epsilon g^{T} \epsilon=g-\operatorname{Tr} g
$$

we obtain

$$
\Delta_{A B}=\Delta_{A} \delta_{A B}
$$

where $\Delta_{A}$ is defined as follows:

$$
\Delta_{A}=(-\Delta,+\Delta,+\Delta,+\Delta, 0,0) .
$$

Therefore the effective lagrangian for free quarks in the $2 \mathrm{SC}$ model can be written as follows

$$
\mathcal{L}_{0}+\mathcal{L}_{\Delta}=\sum_{\vec{v}} \sum_{A=0}^{5} \chi^{A \dagger}\left(\begin{array}{cc}
i V \cdot \partial & \Delta_{A} \\
\Delta_{A} & i \tilde{V} \cdot \partial
\end{array}\right) \chi^{A}+(L \rightarrow R)
$$

From this equation one can immediately obtain the free fermion propagator that in momentum space is still given by (2.40), with the $\Delta_{A}$ given by (2.50). 


\subsection{NGB and their parameters}

In the CFL model the diquark condensate breaks $S U(3)_{L} \otimes S U(3)_{R} \otimes S U(3)_{c} \otimes$ $U(1)_{B}$ to $S U(3)_{c+L+R} \otimes Z_{2}$. By the Goldstone theorem we have 8 NambuGoldstone Bosons +1 related to the breaking of $U(1)_{B}$. We have described the effective theory for this case in par. 1.5. On the other hand in the $2 \mathrm{SC}$ model there is only one would-be NGB associated to the axial color because the chiral group is unbroken and there is an unbroken subgroup that plays the role of $U(1)_{B}$.

How to describe the NGB couplings to the fermions? In order to derive an effective low energy lagrangian for the NGBs and obtain these couplings we use the gradient expansion (for a clear exposition see e.g. [52]). First we introduce as many external fields as the NGBs, with the same quantum numbers of the goldstones; subsequently we perform a derivative expansion of the generating functional. This gives rise to the effective action for the NGBs.

\subsubsection{Gradient expansion for the $U(1)$ NGB in the CFL model and in the 2SC model}

To describe the procedure of the gradient expansion we consider the effective lagrangian for the NGB associated to the spontaneous breaking of the $U(1)$ symmetry. For pedagogical purposes this calculation will be described in detail.

To start with we consider the CFL model. The method of gradient or derivative expansion proceeds as follows. Let us introduce the external field $\sigma$ by the substitution

$$
\chi=\left(\begin{array}{c}
\psi_{+} \\
C \psi_{-}^{*}
\end{array}\right) \rightarrow\left(\begin{array}{c}
U^{\dagger} \psi_{+} \\
U C \psi_{-}^{*}
\end{array}\right)
$$

where

$$
U=e^{i \sigma / f_{\sigma}}
$$

The quark lagrangian (2.39) becomes

$$
\mathcal{L}_{0}+\mathcal{L}_{\Delta}+\mathcal{L}_{\sigma}=\sum_{\vec{v}} \sum_{A=1}^{9} \chi^{A \dagger}\left(\begin{array}{cc}
i V \cdot \partial & \Delta_{A} U^{2} \\
\Delta_{A} U^{\dagger 2} & i \tilde{V} \cdot \partial
\end{array}\right) \chi^{A}+(L \rightarrow R) .
$$

At the lowest order in the field $\sigma$ we have

$$
\mathcal{L}_{\sigma} \approx \sum_{\vec{v}} \sum_{A=1}^{9} \chi^{A \dagger} \Delta_{A}\left(\begin{array}{cc}
0 & \frac{2 i \sigma}{f_{\sigma}}-\frac{2 \sigma^{2}}{f_{\sigma}^{2}} \\
-\frac{2 i \sigma}{f_{\sigma}}-\frac{2 \sigma^{2}}{f_{\sigma}^{2}} &
\end{array}\right) \chi^{A}+(L \rightarrow R)
$$

which contains the couplings $\sigma \chi \chi$ and $\sigma \sigma \chi \chi$. Since $\sigma$ is an external field there is no associated kinetic term. However such term is generated by quantum corrections. To show this we consider the generating functional depending on the external fermion sources $\eta$ and $\eta^{\dagger}$ (we consider only left-handed fields for simplicity):

$$
W\left[\eta, \eta^{\dagger}\right]=\int[d \chi]\left[d \chi^{\dagger}\right][d \sigma] \exp \left\{i \sum_{\vec{v}} \int \chi^{\dagger} A \chi+\eta^{\dagger} \chi+\chi^{\dagger} \eta\right\}
$$


where we have introduced

$$
A=S^{-1}+\frac{2 i \sigma \Delta}{f_{\sigma}} \Gamma_{0}-\frac{2 \sigma^{2} \Delta}{f_{\sigma}^{2}} \Gamma_{1}
$$

and

$$
\Gamma_{0}=\left(\begin{array}{cc}
0 & +1 \\
-1 & 0
\end{array}\right), \quad \Gamma_{1}=\left(\begin{array}{ll}
0 & 1 \\
1 & 0
\end{array}\right)
$$

Clearly we have

$$
\begin{aligned}
W\left[\eta, \eta^{\dagger}\right]=\int[d \chi]\left[d \chi^{\dagger}\right][d \sigma] \times \\
\times \exp \left\{i \sum_{\vec{v}} \int\left(\chi^{\dagger}+\eta^{\dagger} A^{-1}\right) A\left(\chi+A^{-1} \eta\right)-\eta^{\dagger} A^{-1} \eta\right\} .
\end{aligned}
$$

After a change of variables in the functional integration we obtain

$$
\begin{aligned}
& W\left[\eta, \eta^{\dagger}\right]=\int[d \chi]\left[d \chi^{\dagger}\right][d \sigma] \exp \left\{i \sum_{\vec{v}} \int \chi^{\dagger} A \chi-\eta^{\dagger} A^{-1} \eta\right\} \\
= & \int[d \sigma] \exp \left\{-i \sum_{\vec{v}} \int \eta^{\dagger} A^{-1} \eta\right\} \int[d \chi]\left[d \chi^{\dagger}\right] \exp \left\{i \sum_{\vec{v}} \int \chi^{\dagger} A \chi\right\} \\
= & \int[d \sigma] \exp \left\{-i \sum_{\vec{v}} \int \eta^{\dagger} A^{-1} \eta\right\} \exp \{\operatorname{Tr} \ln A\} \\
= & \int[d \sigma] \exp \left\{i \sum_{\vec{v}} \int\left[-i \operatorname{Tr} \ln A-\eta^{\dagger} A^{-1} \eta\right]\right\} .
\end{aligned}
$$

Now we have

$$
\begin{aligned}
& -i \operatorname{Tr} \ln A=-i \operatorname{Tr} \ln S^{-1}\left(1+S \frac{2 i \sigma \Delta}{f_{\sigma}} \Gamma_{0}+S \frac{(-2) \sigma^{2} \Delta}{f_{\sigma}^{2}} \Gamma_{1}\right) \\
& =-i \operatorname{Tr} \ln S^{-1}+i \sum_{n=1}^{\infty} \frac{(-1)^{2 n}}{n}\left(i S \frac{2 i \sigma \Delta}{f_{\sigma}} i \Gamma_{0}+i S \frac{(-2) \sigma^{2} \Delta}{f_{\sigma}^{2}} i \Gamma_{1}\right)^{n} .
\end{aligned}
$$

This is a loop expansion. At the lowest order it produces the effective action

$$
\begin{aligned}
\mathcal{S}_{\text {eff }} & =+\frac{i}{2} \operatorname{Tr} \int d x d y\left[\frac{i S(y, x) 2 i \sigma(x) \Delta}{f_{\sigma}} i \Gamma_{0} \frac{i S(x, y) 2 i \sigma(y) \Delta}{f_{\sigma}} i \Gamma_{0}\right] \\
& +i \operatorname{Tr} \int d x\left[\frac{i S(x, x)(-2) \Delta \sigma^{2}(x)}{f_{\sigma}^{2}} i \Gamma_{1}\right] .
\end{aligned}
$$

The two terms correspond to the diagrams in Fig. 2.1, i.e. the self-energy, Fig. 2.1a, and the tadpole, Fig. 2.1b. They can be computed by a set of Feynman rules to provide

$$
i \mathcal{L}_{\text {eff }}
$$

In momentum space the Feynman rules are as follows: 

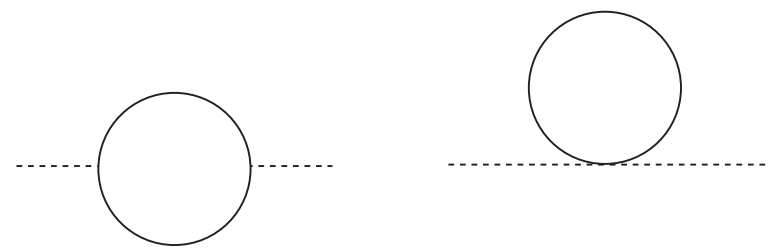

Fig. 2.1 One-loop diagrams. External lines represent the currents $J_{\mu}^{a}, J_{\nu}^{b}$. Full lines are fermion propagators.

1. For each fermionic internal line with momentum $p$ the propagator

$$
i S_{A B}(p)=i \delta_{A B} S(p)=\frac{i \delta_{A B}}{V \cdot p \tilde{V} \cdot p-\Delta_{A}^{2}+i \epsilon}\left(\begin{array}{cc}
\tilde{V} \cdot p & -\Delta_{A} \\
-\Delta_{A} & V \cdot p
\end{array}\right)
$$

2. For each vertex a term $i \mathcal{L}_{\text {int }}$ that can be derived from the effective lagrangian; for example the $\sigma$ couplings to quarks can be derived from (2.55);

3. For each internal momentum not constrained by the momentum conservation the factor

$$
\frac{4 \pi \mu^{2}}{(2 \pi)^{4}} \int d^{2} \ell=\frac{\mu^{2}}{4 \pi^{3}} \int_{-\delta}^{+\delta} d \ell_{\|} \int_{-\infty}^{+\infty} d \ell_{0}
$$

4. A factor of 2 for each fermion loop to take into account the spin $(L+R)$;

5. A statistical factor arising from the Wick theorem if needed.

The result of the calculation of the effective lagrangian in momentum space is as follows:

$$
\begin{aligned}
i \mathcal{L}_{I} & =2 \frac{1}{2} \frac{\mu^{2}}{4 \pi^{3}} \sum_{\vec{v}, A, B} \int d^{2} \ell \\
& \times \operatorname{Tr}\left[i S_{A B}(\ell+p) \frac{2 i \Delta_{B} \sigma}{f_{\sigma}} i \Gamma_{0} i S_{B A}(\ell) \frac{2 i \Delta_{A} \sigma}{f_{\sigma}} i \Gamma_{0}\right], \\
i \mathcal{L}_{I I} & =2 \frac{\mu^{2}}{4 \pi^{3}} \sum_{\vec{v}, A, B} \int d^{2} \ell T r\left[i S_{A B}(\ell) \frac{-2 \Delta_{B} \sigma^{2}}{f_{\sigma}^{2}} i \Gamma_{1}\right]
\end{aligned}
$$

corresponding to the two diagrams of Figs. 2.1a and 2.1b respectively. After some computation one has

$$
\begin{gathered}
i \mathcal{L}_{\text {eff }}(p)=i \mathcal{L}_{I}(p)+i \mathcal{L}_{I I}(p)=\sum_{\vec{v}, A} \frac{\mu^{2} \Delta_{A}^{2}}{\pi^{3} f_{\sigma}^{2}} \\
\times \quad \int d^{2} \ell\left[\frac{\tilde{V} \cdot(\ell+p) \sigma V \cdot \ell \sigma+V \cdot(\ell+p) \sigma \tilde{V} \cdot \ell \sigma-2 \Delta_{A}^{2} \sigma^{2}}{D_{A}(\ell+p) D_{A}(\ell)}\right.
\end{gathered}
$$




$$
\left.-\frac{2 \sigma^{2}}{D_{A}(\ell)}\right]
$$

where we have put

$$
D_{A}(p)=V \cdot p \tilde{V} \cdot p-\Delta_{A}^{2}+i \epsilon .
$$

One can immediately notice that

$$
\mathcal{L}_{I}(p=0)+\mathcal{L}_{I I}(p=0)=0 .
$$

This result implies that the scalar $\sigma$ particle has no mass, in agreement with Goldstone's theorem. To get the effective lagrangian in the CFL model at the lowest order in the $\sigma$ momentum we expand the function in $p(|p| \ll \mid \Delta)$ to get, in momentum space

$$
i \mathcal{L}_{e f f}(p)=\sum_{\vec{v}, A} \frac{2 \mu^{2} \Delta_{A}^{4}}{\pi^{3} f_{\sigma}^{2}}(V \cdot p) \sigma(\tilde{V} \cdot p) \sigma I_{2},
$$

where we have defined

$$
I_{2}=\int \frac{d^{2} \ell}{D_{A}^{3}(\ell)}
$$

(for other integrals of this form see the appendix at the end of this section). Since we have

$$
I_{2}=\frac{-i \pi}{2 \Delta_{A}^{4}}
$$

one gets, in the configuration space,

$$
\mathcal{L}_{e f f}(x)=\frac{9 \mu^{2}}{\pi^{2} f_{\sigma}^{2}} \sum_{\vec{v}}(V \cdot \partial \sigma)(\tilde{V} \cdot \partial \sigma) .
$$

Since

$$
\sum_{\vec{v}} V^{\mu} \tilde{V}^{\nu}=\frac{1}{2} \int \frac{d \vec{v}}{4 \pi} V^{\mu} \tilde{V}^{\nu}=\frac{1}{2}\left(\begin{array}{cccc}
1 & 0 & 0 & 0 \\
0 & -\frac{1}{3} & 0 & 0 \\
0 & 0 & -\frac{1}{3} & 0 \\
0 & 0 & 0 & -\frac{1}{3}
\end{array}\right)_{\mu \nu}
$$

we obtain

$$
\mathcal{L}_{e f f}(x)=\frac{9 \mu^{2}}{\pi^{2} f_{\sigma}^{2}} \frac{1}{2}\left(\left(\partial_{0} \sigma\right)^{2}-v_{\sigma}^{2}(\vec{\nabla} \sigma)^{2}\right),
$$

with

$$
v_{\sigma}^{2}=\frac{1}{3}
$$

This kinetic lagrangian has the canonical normalization factor provided

$$
f_{\sigma}^{2}=\frac{9 \mu^{2}}{\pi^{2}} \quad(\mathrm{CFL}),
$$

which fixes the $\sigma$ constant. Therefore the effective lagrangian for the NGB $\sigma$ particle is:

$$
\mathcal{L}_{e f f}=\frac{1}{2}\left(\left(\partial_{0} \sigma\right)^{2}-\frac{1}{3}(\vec{\nabla} \sigma)^{2}\right),
$$


We note that the value of the velocity (2.74) is a consequence of the average over the Fermi velocities and reflects the number of the space dimensions, i.e. 3. Therefore it is universal and we expect the same value in all the calculations of this type.

In the case of the 2SC model the formalism applies with obvious changes. In particular again we introduce the external field $\sigma$ as in 2.53); the effective quark-NGB lagrangian is given by the analogue of (2.39) where now the sum over $A$ runs from $A=0$ to $A=5$ and $\Delta_{A}$ is given by (2.50). The final result differs from the CFL case only in the coefficient in front of (2.75) which reflects the number of color-flavor gapped degrees of freedom, 9 in the CFL case and 4 in the 2SC case; therefore one has

$$
f_{\sigma}^{2}=\frac{4 \mu^{2}}{\pi^{2}} \quad(2 \mathrm{SC})
$$

whereas the result (2.74) being universal holds also in this case. The NGB effective lagrangian is still given by 2.76). The NGB boson is in this case only a would-be NGB because the axial color is explicitly broken, though this breaking is expected to be small at high densities.

\subsubsection{Gradient expansion for the $S U(3)$ NGBs}

The gradient expansion for the $S U(3)$ NGBs is more complicated because of the color-flavor structure of these fields. However, the procedure is conceptually identical to the case of $U(1)$ : the NGBs are introduced as external fields and acquire a kinetic term, thus becoming dynamical fields, by integrating out the fermion fields. Let us outline this calculation in the present section.

From the invariant coupling

$$
-\frac{\Delta}{2} \sum_{I=1,3} \psi^{T} C \epsilon_{I} \psi \epsilon_{I}
$$

the coupling of the left-handed Weyl spinors $\psi$ 's to the octet of external scalar fields is:

$$
-\frac{\Delta}{2} \sum_{I=1,3} \operatorname{Tr}\left[\left(\psi X^{\dagger}\right)^{T} C \epsilon_{I}\left(\psi X^{\dagger}\right) \epsilon_{I}\right]
$$

with an analogous relation (with matrix $Y$ ) for the right handed fields. Both $X$ and $Y$ have v.e.v. given by

$$
\langle X\rangle=\langle Y\rangle=1
$$

and shall use the gauge

$$
X=Y^{\dagger} .
$$

The lagrangian giving the the coupling of the quarks to the external fields can be obtained from (2.32) and is given by

$$
\mathcal{L}_{D}=\sum_{\vec{v}} \sum_{A, B=1}^{9} \chi^{A \dagger}\left(\begin{array}{cc}
i V \cdot \partial \delta_{A B} & \Xi_{B A}^{*} \\
\Xi_{A B} & i \tilde{V} \cdot \partial \delta_{A B}
\end{array}\right) \chi^{B}
$$




$$
+(L \rightarrow R)
$$

where

$$
\begin{aligned}
\Xi_{A B} & =\Delta \operatorname{Tr}\left[\epsilon_{I}\left(T_{A} X^{\dagger}\right)^{T} \epsilon_{I}\left(T_{B} X^{\dagger}\right)\right]= \\
& =\Delta\left(\operatorname{Tr}\left[T_{A} X^{\dagger} T_{B} X^{\dagger}\right]-\operatorname{Tr}\left[T_{A} X^{\dagger}\right] \operatorname{Tr}\left[T_{B} X^{\dagger}\right]\right) .
\end{aligned}
$$

One can now expand the NGB fields

$$
X=\exp i\left(\frac{\lambda_{a} \Pi^{a}}{2 F}\right), \quad a=1, \cdots, 8,
$$

and obtain the 3-point $\chi \chi \Pi$, and the 4-point $\chi \chi \Pi \Pi$ couplings. The result is, for the 3 -point coupling

$$
\begin{aligned}
\mathcal{L}_{\chi \chi \Pi}= & \sum_{\vec{v}} \frac{-i \Delta}{F}\left\{\sum_{a=1}^{8} \frac{\Pi^{a}}{\sqrt{6}}\left[\chi^{9 \dagger} \Gamma_{0} \chi^{a}+\chi^{a \dagger} \Gamma_{0} \chi^{9}\right]-\right. \\
& \left.-\sum_{a, b, c=1}^{8} d_{a b c} \chi^{a \dagger} \Gamma_{0} \chi^{b} \Pi^{c}\right\} .
\end{aligned}
$$

On the the other hand the 4-point coupling (2 Goldstones +2 fermions)

$$
\begin{aligned}
\mathcal{L}_{\chi \chi \Pi \Pi}= & \sum_{\vec{v}}\left\{\frac{4}{3} \sum_{a=1}^{8} \frac{\Delta}{8 F^{2}} \chi^{9 \dagger} \Gamma_{1} \chi^{9} \Pi^{a} \Pi^{a}\right. \\
& +3 \sqrt{\frac{2}{3}} \sum_{a, b, c=1}^{8}\left(\frac{\Delta}{8 F^{2}} d_{a b c} \chi^{c \dagger} \Gamma_{1} \chi^{9} \Pi^{a} \Pi^{b}+\text { h.c. }\right) \\
& \left.+\sum_{a, b, c, d=1}^{8} \frac{\Delta}{8 F^{2}} h_{a b c d} \chi^{c \dagger} \Gamma_{0} \chi^{d} \Pi^{a} \Pi^{b}\right\} \\
h_{a b c d}= & 2 \sum_{p=1}^{8}\left(g_{c a p} g_{d b p}+d_{c d p} d_{a b p}\right)-\frac{8}{3} \delta_{a c} \delta_{d b}+\frac{4}{3} \delta_{c d} \delta_{a b} .
\end{aligned}
$$

Using the Feynman rules given above and the interaction lagrangians (2.82) and 2.83 we get the effective lagrangian as follows:

$$
\mathcal{L}_{e f f}^{k i n}=\frac{\mu^{2}(21-8 \ln 2)}{36 \pi^{2} F^{2}} \frac{1}{2} \sum_{a=1}^{8}\left(\dot{\Pi}^{a} \dot{\Pi}^{a}-\frac{1}{3}\left|\vec{\nabla} \Pi_{a}\right|^{2}\right) .
$$

The canonical normalization for the kinetic term is obtained provided

$$
F^{2}=\frac{\mu^{2}(21-8 \ln 2)}{36 \pi^{2}}
$$

The NGB velocity is again $1 / \sqrt{3}$ because of the universality noted above. 


\subsection{Gluon masses and dispersion laws}

\subsubsection{Evaluating the bare gluon mass}

Let us go back to Eq. (2.27). $\mathcal{L}_{2}$ is a non local lagrangian containing an infinite number of local terms; let us discuss in the formalism of the two component fermion field $\chi$ introduced in eq. (2.30):

$$
\chi=\left(\begin{array}{c}
\psi_{+} \\
C \psi_{-}^{*}
\end{array}\right)
$$

Let us work in the quark basis $\chi \equiv \chi_{\alpha j},(\alpha=$ color, $j=$ flavor $) ; \mathcal{L}_{2}$ can be written as follows:

$$
\mathcal{L}_{2}=-\sum_{\vec{v}} \chi^{\dagger}\left(\begin{array}{cc}
\{\mu+i \tilde{V} \cdot D\}^{-1} & 0 \\
0 & \{2 \mu+i V \cdot D\}^{-1}
\end{array}\right) P^{\mu \nu} D_{\mu} D_{\nu} \chi .
$$

Let us consider this coupling in the momentum space where

$$
\{2 \mu+i \tilde{V} \cdot D\}^{-1} P^{\mu \nu} D_{\mu} D_{\nu}=\frac{\left(-i \ell_{\mu}+g A_{\mu}^{c} \lambda^{c} / 2\right)\left(-i \ell_{\nu}+g A_{\nu}^{d} \lambda^{d} / 2\right)}{2 \mu+i \tilde{V} \cdot\left(-i \ell+g A^{e} \lambda^{e} / 2\right)} P^{\mu \nu}
$$

Since the effective theory is bidimensional we can put $\ell_{\perp}^{\mu}=\ell_{\rho} P^{\rho \mu}=0$. The simplest coupling generated by this interaction term is a quadrilinear coupling (two quarks, two gluons); it is obtained by putting to zero the coupling to the gluon in the denominator and one gets:

$$
\mathcal{L}_{\chi \chi A A}=-g^{2} \sum_{\vec{v}} \chi^{\dagger} A_{\mu}^{c} A_{\nu}^{d} \frac{\lambda^{c}}{2} \frac{\lambda^{d}}{2}\left(\begin{array}{cc}
\frac{1}{2 \mu+\tilde{V} \cdot \ell} & 0 \\
0 & \frac{1}{2 \mu+V \cdot \ell}
\end{array}\right) P^{\mu \nu} \chi .
$$

Let us now consider the CFL model. Introducing the quark basis $\chi^{A}$ ( $A=$ $1, \ldots, 9)$, we get the coupling in the following form:

$$
\mathcal{L}_{\chi \chi A A}=-g^{2} \sum_{\vec{v}} \chi_{A}^{\dagger} A_{\mu}^{c} A_{\nu}^{d}\left(\begin{array}{cc}
\frac{1}{2 \mu+\tilde{V} \cdot \ell} & 0 \\
0 & \frac{1}{2 \mu+V \cdot \ell}
\end{array}\right) P^{\mu \nu} \chi_{B} \xi_{c d}^{A B},
$$

where the Clebsch Gordan coefficient is given by

$$
\xi_{c d}^{A B}=\frac{1}{8} \operatorname{tr}\left(\lambda^{A} \lambda^{c} \lambda^{d} \lambda^{D}\right)
$$

$\mathcal{L}_{\chi \chi A A}$ contributes to the gluon self-energy through a tadpole graph analogous to that in Fig. 2.1b (the external lines should be interpreted now as gluon lines). It is important to stress that, due to the denominator in (2.89), the loop integral takes relevant contribution from the region where $\ell_{0} \pm \ell_{\|} \approx-2 \mu$. This means that one cannot integrate only near the Fermi surface, but the longitudinal integration must be extended up to $2 \mu$ or, in terms of $\ell_{\|}=p-\mu$, from $-\mu$ to $+\mu$. 
The tadpole contribution to the gluon self energy can be computed by using the set of Feynman rules discussed above and by (2.89). One gets

$$
\begin{gathered}
\Pi_{c d}^{\mu \nu}=-i 4 g^{2} \sum_{\vec{v}} P^{\mu \nu} \int_{-\mu}^{+\mu} \frac{d \ell_{\|}}{2 \pi}\left(\mu+\ell_{\|}\right)^{2} \int_{-\infty}^{+\infty} \frac{d \ell_{0}}{2 \pi} \times \\
\times\left(\sum_{A=1}^{8} \xi_{c d}^{A A} \frac{1}{8 D(\ell)}+\frac{\delta_{c d}}{6 D^{\prime}(\ell)}\right) \cdot\left(\frac{\tilde{V} \cdot \ell}{2 \mu+\tilde{V} \cdot \ell+i \epsilon}+\tilde{V} \rightarrow V\right)
\end{gathered}
$$

where $D(\ell)=\ell_{0}^{2}-\ell_{\|}^{2}-\Delta^{2}+i \epsilon$ and $D^{\prime}(\ell)=\ell_{0}^{2}-\ell_{\|}^{2}-\Delta^{\prime 2}+i \epsilon$. The factor $4=2 \times 2$ originates from a statistical factor and a spin factor. Since

$$
\sum_{A=1}^{8} \xi_{c d}^{A A}=\frac{4}{3} \delta_{c d}
$$

and

$$
\sum_{\vec{v}} P^{\mu \nu}=-\frac{1}{3} \delta^{\mu \nu} \delta^{\mu i} \delta^{\nu j}
$$

we obtain

$$
\begin{aligned}
& \Pi_{c d}^{00}=0, \quad \Pi_{c d}^{0 j}=0, \\
& \Pi_{c d}^{i j}=-\omega_{p}^{2} \delta^{i j} \delta_{c d}
\end{aligned}
$$

where $\omega_{p}=g \mu / \sqrt{2} \pi$ is the plasma frequency and we have used the result

$$
\int_{-\mu}^{+\mu} \frac{d \ell_{\|}}{2 \pi}\left(\mu+\ell_{\|}\right)^{2} \int_{-\infty}^{+\infty} \frac{d \ell_{0}}{2 \pi} \frac{\tilde{V} \cdot \ell}{(2 \mu+\tilde{V} \cdot \ell+i \epsilon)\left(\ell_{0}^{2}-\ell_{\|}^{2}-\Delta^{2}+i \epsilon\right)}=\frac{i \mu^{2}}{8 \pi} .
$$

For the 2SC case the calculation is similar; one obtains the following results 目 $(c, d=1, \cdots, 8)$ :

$$
\begin{aligned}
& \Pi_{c d}^{00}=0, \quad \Pi_{c d}^{0 j}=0 \\
& \Pi_{c d}^{i j}=-m_{g}^{2} \delta^{i j} \delta_{c d}
\end{aligned}
$$

where $m_{g}=g \mu / \sqrt{3} \pi$. Note that the tadpole graphs only contributes to the Meissner mass.

\subsubsection{Debye and Meissner mass and gluon approximate disper- sion laws: CFL case}

For both the CFL and the 2SC model the calculation of Section 2.4.1 provides a mass term to the gluons; it is a chromo-magnetic mass term (Meissner mass)

\footnotetext{
${ }^{3}$ We note that in this case the tadpole diagram presents infrared divergences (in $\ell_{0}$ ). To control these divergences one considers the system in a heat bath and substitutes the energy euclidean integration $\ell_{4}=-i \ell_{0}$ with a sum over the Matsubara frequencies $\ell_{4} \rightarrow \omega_{n}=$ $2 \pi\left(n+\frac{1}{2}\right) T$; eventually one performs the limit $T \rightarrow 0$. For details see 31 and the appendix.
} 
since it gives mass to $\vec{A}$; there is however a second diagram contributing to the Meissner mass, i.e. the graph of Fig. 2.1a, with external line interpreted as a gluon. As we shall see in this section it contributes also to the chromo-electric (Debye) mass and to the dispersion law, i.e.

$$
E=E(p)
$$

masses and dispersion laws can be derived by the polarization tensor

$$
\Pi^{\mu \nu}(p)
$$

For example

$$
\begin{aligned}
\Pi^{00}(0) & =m_{D}^{2} \\
\Pi^{i j}(0) & =-\delta^{i j} m_{M}^{2} .
\end{aligned}
$$

To compute $\Pi^{\mu \nu}$ from Fig. 2.1a we need $\mathcal{L}_{1}$ i.e. the interaction term between the quarks and one gluon. The fermionic current could be extracted from eq. (2.26). However it is more convenient to work in the quark basis $\chi^{A}$. We use eq. (2.32) to write, in the CFL case

$$
\mathcal{L}_{1}=i g A_{a}^{\mu} J_{\mu}^{a}
$$

where

$$
J_{\mu}^{a}=\sum_{\vec{v}} \sum_{A, B=1}^{9} \chi^{A \dagger}\left(\begin{array}{cc}
i V_{\mu} h_{A a B} & 0 \\
0 & -i \tilde{V}_{\mu} h_{A a B}^{*}
\end{array}\right) \chi^{B}+(L \rightarrow R)
$$

where

$$
h_{A a B}=\frac{1}{2} \operatorname{Tr}\left[T_{A} \lambda_{a} T_{B}\right]
$$

We obtain

$$
\begin{aligned}
J_{\mu}^{a} & =\frac{i}{2} \sqrt{\frac{2}{3}} \sum_{\vec{v}}\left(\chi^{9 \dagger}\left(\begin{array}{cc}
V_{\mu} & 0 \\
0 & -\tilde{V}_{\mu}
\end{array}\right) \chi^{a}+\text { h.c. }\right)+ \\
& +\frac{i}{2} \sum_{\vec{v}} \sum_{b, c=1}^{8} \chi^{b \dagger}\left(\begin{array}{cc}
V_{\mu} g_{b a c} & 0 \\
0 & -\tilde{V}_{\mu} g_{b a c}^{*}
\end{array}\right) \chi^{c}
\end{aligned}
$$

where

$$
g_{a b c}=d_{a b c}+i f_{a b c} .
$$

and $d_{a b c}, f_{a b c}$ are the usual $S U(3)$ symbols. The result of the self energy diagram (Fig. 2.1a, with external lines representing fermionic currents) can be written as follows:

$$
\begin{gathered}
i \Pi_{a b}^{\mu \nu}(p)=-2 \sum_{\vec{v}} \sum_{A, C, D, E}\left(\frac{-i g}{2}\right)^{2} \frac{4 \pi \mu^{2}}{(2 \pi)^{4}} \int d^{2} \ell \operatorname{Tr}\left[i S_{C D}(\ell+p) \times\right. \\
\left.\times\left(\begin{array}{cc}
V_{\nu} h_{D b E} & 0 \\
0 & -\tilde{V}_{\nu} h_{D b E}^{*}
\end{array}\right) i S_{E A}(\ell)\left(\begin{array}{cc}
V_{\mu} h_{A a C} & 0 \\
0 & -\tilde{V}_{\mu} h_{A a C}^{*}
\end{array}\right)\right]
\end{gathered}
$$


where the propagator is given by eq. (2.63). We note the minus sign on the r.h.s of (2.114), due to the presence of a fermion loop and the factor 2 due to the spin $(L+R)$. To this result one should add the contribution arising from the tadpole diagram of Fig. 2.1b (with, also in this case, external lines representing fermionic currents $J_{\mu}^{a}, J_{\nu}^{b}$ ); this diagram was computed in 2.4.1:

$$
\left.\Pi_{a b}^{\mu \nu}\right|_{1 b}=-\delta_{a b} \delta^{j k} \delta^{\mu j} \delta^{\nu k} \omega_{p}^{2} .
$$

To derive the dispersion law for the gluons, we write the equations of motion for the gluon field $A_{\mu}^{b}$ in momentum space and high-density limit:

$$
\left[\left(-p^{2} g^{\nu \mu}+p^{\nu} p^{\mu}\right) \delta_{a b}+\Pi_{a b}^{\nu \mu}\right] A_{\mu}^{b}=0 .
$$

We define the invariant quantities $\Pi_{0}, \Pi_{1}, \Pi_{2}$ and $\Pi_{3}$ by means of the following equations,

$$
\Pi^{\mu \nu}\left(p_{0}, \vec{p}\right)=\left\{\begin{array}{l}
\Pi^{00}=\Pi_{0}\left(p_{0}, \vec{p}\right) \\
\Pi^{0 i}=\Pi^{i 0}=\Pi_{1}\left(p_{0}, \vec{p}\right) n^{i} \\
\Pi^{i j}=\Pi_{2}\left(p_{0}, \vec{p}\right) \delta^{i j}+\Pi_{3}\left(p_{0}, \vec{p}\right) n^{i} n^{j}
\end{array}\right.
$$

with $\vec{n}=\frac{\vec{p}}{p}$. It is clear that in deriving the dispersion laws we cannot go beyond momenta at most of the order $\Delta$, as the Fermi velocity superselection rule excludes gluon exchanges with very high momentum; it is therefore an approximation, but nevertheless a useful one, as in most cases hard gluon exchanges are strongly suppressed by the asymptotic freedom property of QCD.

Besides the longitudinal and transverse gluon fields defined by

$$
\begin{aligned}
A_{i L}^{a} & =\left(\vec{n} \cdot \vec{A}^{a}\right) n_{i}, \\
A_{i T}^{a} & =A_{i}^{a}-A_{i L}^{a},
\end{aligned}
$$

it is useful to consider also the scalar gluon field $A_{0}^{a}$. By the equation

$$
p_{\nu} \Pi_{a b}^{\nu \mu} A_{\mu}^{b}=0
$$

one obtains the relation

$$
\left(p_{0} \Pi_{0}-|\vec{p}| \Pi_{1}\right) A_{0}=\vec{n} \cdot \vec{A}\left(p_{0} \Pi_{1}-|\vec{p}|\left(\Pi_{2}+\Pi_{3}\right)\right),
$$

between the scalar and the longitudinal component of the gluon fields. The dispersion laws for the scalar, longitudinal and transverse gluons are respectively

$$
\begin{aligned}
\left(\Pi_{2}+\Pi_{3}+p_{0}^{2}\right)\left(|\vec{p}|^{2}+\Pi_{0}\right) & =p_{0}|\vec{p}|\left(2 \Pi_{1}+p_{0}|\vec{p}|\right), \\
\left(\Pi_{2}+\Pi_{3}+p_{0}^{2}\right)\left(|\vec{p}| p_{0}+\Pi_{0}\right) & =p_{0}|\vec{p}|\left(2 \Pi_{1}+p_{0}^{2}\right)+\Pi_{1}^{2}, \\
p_{0}^{2}-|\vec{p}|^{2}+\Pi_{2} & =0 .
\end{aligned}
$$

The analysis of these equations is rather complicated [53]. We give an approximate evaluation of them by developing $\Pi^{\mu \nu}$ in powers of $p$. By including in the previous formulas terms up to the second order in momenta we get [29]:

$$
\left.\Pi_{a b}^{\mu \nu}(p)\right|_{1 a}=\Pi_{a b}^{\mu \nu}(0)+\delta \Pi_{a b}^{\mu \nu}(p)
$$


where

$$
\begin{aligned}
& \Pi_{a b}^{\mu \nu}(0)=\frac{\mu^{2} g^{2}}{2 \pi} \delta_{a b} \sum_{\vec{v}} \Sigma^{0, \mu \nu}, \\
& \delta \Pi_{a b}^{\mu \nu}(p)=\frac{\mu^{2} g^{2}}{2 \pi} \delta_{a b} \sum_{\vec{v}} \Sigma^{\mu \nu},
\end{aligned}
$$

and

$$
\begin{gathered}
\Sigma^{0, \mu \nu}=k_{1}\left(V^{\mu} V^{\nu}\right)+k_{2}\left(V^{\mu} \tilde{V}^{\nu}\right)+(V \rightarrow \tilde{V}), \\
\Sigma^{\mu \nu}=a V^{\mu} V^{\nu} \frac{(\tilde{V} \cdot p)^{2}}{\Delta^{2}}+b V^{\mu} \tilde{V}^{\nu} \frac{V \cdot p \tilde{V} \cdot p}{\Delta^{2}}+(V \rightarrow \tilde{V}) .
\end{gathered}
$$

The coefficients are given by

$$
\begin{aligned}
a & =\frac{1}{108 \pi}\left(31-\frac{32}{3} \ln 2\right) \\
b & =\frac{10}{108 \pi}\left(1-\frac{8}{3} \ln 2\right) \\
k_{1} & =\frac{3}{2 \pi} \\
k_{2} & =-\frac{1}{9 \pi}(3+4 \ln 2) .
\end{aligned}
$$

Since one has, including the one-loop $\mathcal{O}\left(g^{2}\right)$ correction in the gluonic lagrangian,

$$
\mathcal{L}_{G}=-\frac{1}{4} G^{2} \rightarrow-\frac{1}{4} G^{2}+\frac{1}{2} A_{a \nu} \Pi_{a b}^{\mu \nu} A_{b \mu},
$$

one can compute the one-loop contributions to the Debye and Meissner mass from $\Pi_{a b}^{00}(0)$ and $\Pi_{a b}^{i j}(0)$ respectively, according to 2.100); to the results of (2.115) for the diagram of Fig. 2.1a, one must add the contribution (2.107); the two masses are identical for all the eight gluons in the CFL model and are given by:

$$
m_{D}^{2}=\frac{\mu^{2} g^{2}}{36 \pi^{2}}(21-8 \ln 2)=g^{2} F^{2}
$$

and

$$
m_{M}^{2}=\frac{\mu^{2} g^{2}}{\pi^{2}}\left(-\frac{11}{36}-\frac{2}{27} \ln 2+\frac{1}{2}\right)=\frac{m_{D}^{2}}{3}
$$

where the first two terms are the result of the diagram of Fig.2.1a and the last one is the result of the diagram of Fig. 2.1b (this contribution is called the bare Meissner mass in [45]). These results have been obtained by the method of the effective lagrangian in [29] and agree with the findings of other authors 45], [46]. In particular $m_{M}^{2}=v^{2} m_{D}^{2}$, where $v$, as for the NGB velocity, is equal to $1 / \sqrt{3}$.

The Debye and Meissner masses do not exhaust the analysis of the dispersion laws for the gluons in the medium. In particular we wish to show the existence of a light excitation for small momenta in the CFL phase. To obtain the dispersion 
laws for the gluons in the small momenta limit we proceed as follows. From (2.112) one obtains, for small momenta:

$$
p^{0} A_{0} \approx \frac{1}{3}(\vec{p} \cdot \vec{A}) .
$$

Substituting this result in (2.111) taken for $\nu=0$ one gets the dispersion law for the time-like gluons $A_{0}^{b}$ :

$$
p^{0}= \pm E_{A_{0}}, \quad E_{A_{0}}=\frac{1}{\sqrt{3}} \sqrt{|\vec{p}|^{2}+\frac{m_{D}^{2}}{1+\alpha_{1}}},
$$

with

$$
\alpha_{1}=\frac{\mu^{2} g_{s}^{2}}{6 \Delta^{2} \pi}(a-b)=\frac{\mu^{2} g_{s}^{2}}{216 \Delta^{2} \pi^{2}}\left(7+\frac{16}{3} \ln 2\right) .
$$

The rest mass of $A_{0}^{b}$ is therefore given by

$$
m_{A_{0}}^{R}=\frac{m_{D}}{\sqrt{3\left(1+\alpha_{1}\right)}} .
$$

Since for large values of $\mu$ one has $g^{2} \mu^{2} \gg \Delta^{2}$ it follows that

$$
m_{A_{0}}^{R} \approx \frac{m_{D}}{\sqrt{3 \alpha_{1}}}=\sqrt{6 \frac{21-8 \ln 2}{21+16 \ln 2}} \Delta \approx 1.70 \Delta .
$$

We also define the so-called effective mass (to be denoted $m^{*}$ in the sequel) by

$$
\vec{v}=\frac{\partial E}{\partial \vec{p}}=\frac{\vec{p}}{m^{*}(p)}
$$

in the limit $\vec{p} \rightarrow 0$. We get, if $m^{*}=m^{*}(0)$,

$$
m_{A_{0}}^{*}=\frac{\sqrt{3} m_{D}}{\sqrt{1+\alpha_{1}}}=3 m_{A_{0}}^{R} \approx 5.10 \Delta
$$

Let us now turn to the dispersion law for the longitudinal and transverse gluons; they are obtained by considering (2.111) for $\nu=i$ and use again (2.126). One gets the dispersion laws for the results for the longitudinal and transverse cases as follows:

$$
\begin{aligned}
E_{A_{L}} & =\frac{1}{\sqrt{3}} \sqrt{|\vec{p}|^{2} \frac{1+\alpha_{2}}{1+\alpha_{1}}+\frac{m_{D}^{2}}{1+\alpha_{1}}} \\
E_{A_{T}} & =\sqrt{|\vec{p}|^{2} \frac{1+\alpha_{3}}{1+\alpha_{1}}+\frac{m_{D}^{2}}{3\left(1+\alpha_{1}\right)}},
\end{aligned}
$$

with

$$
\alpha_{2}=\frac{\mu^{2} g_{s}^{2}}{30 \Delta^{2} \pi^{2}}(a-9 b)=-\frac{\mu^{2} g_{s}^{2}}{3240 \Delta^{2} \pi^{2}}\left(59-\frac{688}{3} \ln 2\right)
$$




$$
\alpha_{3}=-\frac{\mu^{2} g_{s}^{2}}{30 \Delta^{2} \pi^{2}}(a+b)=-\frac{\mu^{2} g_{s}^{2}}{3240 \Delta^{2} \pi^{2}}\left(41-\frac{112}{3} \ln 2\right)
$$

We get easily the following results

$$
m_{A_{L}}^{R}=m_{A_{T}}^{R}=m_{A_{0}}^{R}=\frac{m_{D}}{\sqrt{3\left(1+\alpha_{1}\right)}} \equiv m_{R} .
$$

The results (2.130) and 2.135) show that the rest mass is of of the order of $\Delta$ and one could therefore wonder if these results are significant, since they have been obtained in the limit $|p / \Delta| \ll 1$. To estimate the validity of this approximation we use the exact result, which can be obtained by equations (2.113). Since $\Pi_{3}\left(p_{0}, 0\right)=0$, the rest mass of the three species $A_{0}, A_{L}, A_{T}$ is given by

$$
m^{2}+\Pi_{2}\left(m^{2}, 0\right)=0
$$

with

$$
\begin{aligned}
\Pi_{2}\left(m^{2}, 0\right) & =\frac{\mu^{2} g^{2}}{2 \pi^{2}}\left[-1+\int_{0}^{+\infty} d x\left(\frac{1}{e(x)\left[4 e(x)^{2}-m^{2} / \Delta^{2}\right]}\right.\right. \\
& \left.\left.+\frac{e(x)+\tilde{e}(x)}{9 e(x) \tilde{e}(x)} \frac{2-x^{2}+e(x) \tilde{e}(x)}{(e(x)+\tilde{e}(x))^{2}-m^{2} / \Delta^{2}}\right)\right]
\end{aligned}
$$

with

$$
\begin{aligned}
& e(x)=\sqrt{1+x^{2}} \\
& \tilde{e}(x)=\sqrt{4+x^{2}} .
\end{aligned}
$$

The numerical result of (2.136) is

$$
m \equiv m_{R}=1.36 \Delta .
$$

A comparison with (2.130) shows that the relative error between the two procedures is of the order of $18 \%$ and this is also the estimated difference for the dispersion law at small $\vec{p}$. We notice that the gradient expansion approximation tends to overestimate the correct result [53].

\subsubsection{Debye and Meissner mass and gluon approximate disper- sion laws: 2SC case}

The analysis of the dispersion laws for the glue in the case of the 2SC model is complicated by the presence of an unbroken color subgroup.

The fermionic current appearing in

$$
\mathcal{L}_{1}=i g A_{\mu}^{a} J_{a}^{\mu}
$$

can be derived from 2.45). One finds

$$
J_{\mu}^{a}=\sum_{\vec{v}} \sum_{A, B=0}^{5} \chi^{A \dagger}\left(\begin{array}{cc}
i V_{\mu} K_{A a B} & 0 \\
0 & -i \tilde{V}_{\mu} K_{A a B}^{*}
\end{array}\right) \chi^{B}+(L \rightarrow R) .
$$


where the coefficients $K_{A a B}$ are given by:

$$
K_{A a B}=\frac{1}{2} \operatorname{Tr}\left\{\tilde{\lambda}_{A} \lambda^{a} \tilde{\lambda}_{B}\right\}
$$

In this way one can compute the self energy contribution to $\Pi_{a b}^{\mu \nu}$. These results are contained in [31] and we briefly summarize them.

We write

$$
\Pi_{a b}^{\mu \nu}(p)=\Pi_{a b}^{\mu \nu}(0)+\delta \Pi_{a b}^{\mu \nu}(p)
$$

with

$$
\Pi_{a b}^{\mu \nu}(0)=\frac{\mu^{2} g^{2}}{\pi^{2}} \sum_{\vec{v}} \Sigma_{a b}^{0, \mu \nu}
$$

and

$$
\delta \Pi_{a b}^{\mu \nu}(p)=\frac{\mu^{2} g^{2}}{\pi^{2}} \sum_{\vec{v}} \Sigma_{a b}^{\mu \nu}(p) .
$$

It is useful to distinguish gluons of different colours, i.e. the gluons $A_{\mu}^{a}$ ( $a=$ $1,2,3)$ of the unbroken color subgroup $S U(2)_{c}$ and the gluons $A_{\mu}^{a}$ with $a=4, \ldots 7$ and $a=8$.

\section{Colors $=1,2,3$}

In this case one finds from the self energy diagram of Fig. $2.1(a, b=1,2,3)$ :

$$
\begin{aligned}
\Pi_{a b}^{\mu \nu}(p) & =i \delta_{a b} \frac{g^{2} \mu^{2}}{2 \pi^{3}} \sum_{\vec{v}} \int d^{2} \ell\left(\frac{V^{\mu} V^{\nu} \tilde{V} \cdot \ell \tilde{V} \cdot(\ell+p)+(V \leftrightarrow \tilde{V})}{D_{1}(\ell+p) D_{1}(\ell)}+\right. \\
& \left.+\Delta^{2} \frac{V^{\mu} \tilde{V}^{\nu}+V^{\nu} \tilde{V}^{\mu}}{D_{1}(\ell+p) D_{1}(\ell)}\right) .
\end{aligned}
$$

As we noted in the previous sections working out the CFL case the extraction of the dispersion law in the general case is rather difficult. We consider here only the small momentum limit. Working in this limit one gets for the various components of the tensor $\Pi^{\mu \nu}$ :

$$
\begin{gathered}
\Pi_{a b}^{00}(p)=\Pi_{a b}^{00}(0)+\delta \Pi_{a b}^{00}(p)=\delta \Pi_{a b}^{00}(p)=\delta \delta_{a b} \frac{\mu^{2} g^{2}}{18 \pi^{2} \Delta^{2}}|\vec{p}|^{2}, \\
\Pi_{a b}^{k l}(p)=\Pi_{a b}^{k l}(0)+\delta \Pi_{a b}^{k l}(p)=\delta_{a b} \delta^{k l} \frac{\mu^{2} g^{2}}{3 \pi^{2}}\left(1+\frac{p_{0}^{2}}{6 \Delta^{2}}\right),
\end{gathered}
$$

and

$$
\Pi_{a b}^{0 k}(p)=\delta \Pi_{a b}^{0 k}(p)=\delta_{a b} \frac{\mu^{2} g^{2}}{18 \pi^{2} \Delta^{2}} p^{0} p^{k} .
$$

These results agree with the outcomes of [54] and [55].

We note that there is no contribution to the Debye mass in this case; moreover the contribution to the Meissner mass from this term exactly cancels the contribution from the tadpole graph. This results reflects the fact that in the 2SC model the $S U(2)_{c}$ color subgroup generated by the first three generators $T^{c}(c=1,2,3)$ remains unbroken. 
One can now compute the dispersion laws for the unbroken gluons. From the previous formulae one gets:

$$
\mathcal{L}=-\frac{1}{4} F_{a}^{\mu \nu} F_{\mu \nu}^{a}+\frac{1}{2} \Pi_{a b}^{\mu \nu} A_{\mu}^{a} A_{\nu}^{b}
$$

Introducing the fields $E_{i}^{a} \equiv F_{0 i}^{a}$ and $B_{i}^{a} \equiv i \varepsilon_{i j k} F_{j k}^{a}$, and using (2.147), (2.148) and (2.149) these results can be written as follows (we neglect for the time being the 3 gluons and 4 gluons vertices to be discussed below):

$$
\mathcal{L}=\frac{1}{2}\left(E_{i}^{a} E_{i}^{a}-B_{i}^{a} B_{i}^{a}\right)+\frac{k}{2} E_{i}^{a} E_{i}^{a},
$$

with

$$
k=\frac{g^{2} \mu^{2}}{18 \pi^{2} \Delta^{2}} .
$$

These results have been first obtained in [55]. As discussed in this paper, these results imply that the medium has a very high dielectric constant $\epsilon=k+1$ and a magnetic permeability $\lambda=1$. The gluon speed in this medium is now

$$
v=\frac{1}{\sqrt{\epsilon \lambda}} \propto \frac{\Delta}{g \mu}
$$

and in the high density limit it tends to zero. The one loop lagrangian (2.151) assumes the gauge invariant expression

$$
\mathcal{L}=-\frac{1}{4} F_{j}^{\mu \nu} F_{\mu \nu}^{j} \quad(j=1,2,3),
$$

provided the following rescaling is used

$$
\begin{aligned}
A_{0}^{j} & \rightarrow A_{0}^{j \prime}=k^{3 / 4} A_{0}^{j}, \\
A_{i}^{j} & \rightarrow A_{i}^{j \prime}=k^{1 / 4} A_{i}^{j}, \\
x_{0} & \rightarrow x_{0}^{\prime}=k^{-1 / 2} x_{0}, \\
g & \rightarrow g^{\prime}=k^{-1 / 4} g .
\end{aligned}
$$

\section{Colors $=4-7$}

For $a, b=4, \ldots, 7$ the polarization tensor is

$$
\begin{aligned}
\Pi_{a b}^{\mu \nu}(p) & =i \delta_{a b} \frac{g^{2} \mu^{2}}{4 \pi^{3}} \sum_{\vec{v}} \int d^{2} \ell\left(V^{\mu} V^{\nu} \tilde{V} \cdot \ell \tilde{V} \cdot(\ell+p)+V \leftrightarrow \tilde{V}\right) \times \\
& \times\left(\frac{1}{D_{1}(\ell+p) D_{2}(\ell)}+\frac{1}{D_{2}(\ell+p) D_{1}(\ell)}\right) .
\end{aligned}
$$

In the small momentum limit one gets in this case

$$
\Pi_{a b}^{00}(p)=\Pi_{a b}^{00}(0)+\delta \Pi_{a b}^{00}(p)=\delta_{a b} \frac{\mu^{2} g^{2}}{2 \pi^{2}}\left(1+\frac{p_{0}^{2}+|\vec{p}|^{2} / 3}{2 \Delta^{2}}\right),
$$




$$
\Pi_{a b}^{0 i}(p)=\delta \Pi_{a b}^{0 i}(p)=\delta_{a b} \frac{\mu^{2} g^{2}}{6 \pi^{2} \Delta^{2}} p^{0} p^{i}
$$

and

$$
\Pi_{a b}^{i j}(p)=\Pi_{a b}^{i j}(0)+\delta \Pi_{a b}^{i j}(p)=\delta_{a b} \frac{\mu^{2} g^{2}}{6 \pi^{2}}\left(\delta^{i j}+\frac{\delta^{i j} p_{0}^{2}}{2 \Delta^{2}}+\frac{\delta^{i j} \vec{p}^{2}+2 p^{i} p^{j}}{10 \Delta^{2}}\right) .
$$

We can observe a contribution to both the Debye and Meissner mass in this case.

\section{Color $=8$}

For the gluon 8 , one has

$$
\begin{aligned}
\Pi_{88}^{\mu \nu} & =i \frac{g^{2} \mu^{2}}{6 \pi^{3}} \sum_{\vec{v}} \int d^{2} \ell\left[\left(V^{\mu} V^{\nu} \tilde{V} \cdot \ell \tilde{V} \cdot(\ell+p)+V \leftrightarrow \tilde{V}\right) \times\right. \\
& \times\left(\frac{1}{D_{1}(\ell+p) D_{1}(\ell)}+\frac{2}{D_{2}(\ell+p) D_{2}(\ell)}\right) \\
& \left.-\Delta^{2} \frac{V^{\mu} \tilde{V}^{\nu}+V^{\nu} \tilde{V}^{\mu}}{D_{1}(\ell+p) D_{1}(\ell)}\right],
\end{aligned}
$$

and, in the small momentum limit,

$$
\Pi_{88}^{00}(p)=\Pi_{88}^{00}(0)+\delta \Pi_{88}^{00}(p)=\frac{\mu^{2} g^{2}}{\pi^{2}}\left(1+\frac{p_{0}^{2}}{18 \Delta^{2}}\right)
$$

and

$$
\begin{gathered}
\Pi_{88}^{0 i}(p)=\delta \Pi_{88}^{0 i}(p)=\frac{\mu^{2} g^{2}}{54 \pi^{2} \Delta^{2}} p^{0} p^{i} \\
\Pi_{88}^{i j}(p)=\Pi_{88}^{i j}(0)+\delta \Pi_{88}^{i j}(p)=\frac{\mu^{2} g^{2}}{18 \pi^{2}}\left(4 \delta^{i j}+\frac{\delta^{i j} \vec{p}^{2}+2 p^{i} p^{j}}{15 \Delta^{2}}\right) .
\end{gathered}
$$

Also in this case there are contributions to the Debye and Meissner masses. The results are summarized in the following table.

\begin{tabular}{|c|c|c|}
\hline$a$ & $\Pi^{00}(0)$ & $-\Pi^{i j}(0)$ \\
\hline $1-3$ & 0 & 0 \\
\hline $4-7$ & $\frac{3}{2} m_{g}^{2}$ & $\frac{1}{2} m_{g}^{2}$ \\
\hline 8 & $3 m_{g}^{2}$ & $\frac{1}{3} m_{g}^{2}$ \\
\hline
\end{tabular}

Table 2.1: Debye and Meissner masses in the 2SC phase; $m_{g}^{2}=\frac{\mu^{2} g^{2}}{3 \pi^{2}}$.

These results are in agreement with a calculation performed by [54 with a different method. 


\section{Dispersion laws and light plasmons for gluons 4-8}

To get the dispersion law for the broken color gluons we follow the procedure used for the CFL case, Eq. (2.108) and its sequel.

Expanding (2.112) at first order in $p$ we find for $b=\beta=4,5,6,7$

$$
p^{0} A_{0}^{\beta}=\frac{1}{3} \vec{p} \cdot \vec{A}^{\beta},
$$

while for $b=8$

$$
p^{0} A_{0}^{8}=\frac{1}{9} \vec{p} \cdot \vec{A}^{8} .
$$

For colors 4-7 we use eqs. 2.156) - (2.158) and we get the following dispersion laws for the longitudinal and transverse modes:

$$
\begin{aligned}
& E_{L}=\sqrt{-\frac{7}{15}|\vec{p}|^{2}+2 \Delta^{2}}, \\
& E_{T}=\sqrt{-\frac{1}{5}|\vec{p}|^{2}+2 \Delta^{2}},
\end{aligned}
$$

The rest mass for these gluons, in the gradient expansion approximation, is given by

$$
m_{A_{L}}^{R}=m_{A_{T}}^{R}=\sqrt{2} \Delta .
$$

To estimate the validity of this approximation we can use the exact result, obtained analogously to the method described for the CFL case. The rest mass of the three species $A_{0}, A_{L}, A_{T}$ is given by

$$
m^{2}+\Pi_{2}\left(m^{2}, 0\right)=0,
$$

with

$$
\Pi_{2}\left(m^{2}, 0\right)=\frac{\mu^{2} g^{2}}{3 \pi^{2}}\left[-1+\int_{0}^{+\infty} d x \frac{x+\sqrt{x^{2}+1}}{\left(x+\sqrt{x^{2}+1}\right)^{2}-m^{2}}\left(1-\frac{x}{\sqrt{x^{2}+1}}\right)\right]
$$

The numerical result of (2.168) is

$$
m \equiv m_{R}=0.894 \Delta .
$$

A comparison with (2.167) shows that the difference is of the order of $40-50 \%$ and this is also the estimated difference for the dispersion law at small $|\vec{p}|$. We notice that also in this case, as for three flavors, the gradient expansion approximation tends to overestimate the correct result.

In the case of the gluon of color 8 we work as for the colors 4-7. We obtain the following dispersion laws for the longitudinal and transverse modes:

$$
\begin{aligned}
E_{L} & =\sqrt{\frac{4}{270} \frac{m_{M}^{2}}{\Delta^{2}}|\vec{p}|^{2}+m_{M}^{2}} \\
E_{T} & =\sqrt{-\frac{1}{30} \frac{m_{M}^{2}}{\Delta^{2}}|\vec{p}|^{2}+m_{M}^{2}}
\end{aligned}
$$


where the Meissner mass is in table 2.1. From these equations we see that

$$
m_{A_{L}}^{R}=m_{A_{T}}^{R}=m_{M} .
$$

The large rest mass of the 8th gluon is an exact result, independent of the small momentum approximation and is due to the fact that, in this case

$$
\Pi_{2}\left(m^{2}, 0\right)=-\frac{\mu^{2} g^{2}}{9 \pi^{2}}=-m_{M}^{2}
$$

\subsection{One-loop corrections to three- and four-point gluon vertices in the $2 \mathrm{SC}$ model}

To complete the effective lagrangian for the eight gluons in the two-flavor model one can compute the one loop corrections to the gluon vertices $\Gamma_{3}$ (3 gluons) and $\Gamma_{4}$ (4 gluons). We shall not consider in the sequel a possible light glueball which is discussed in [56]. Therefore we write the full gluon lagrangian as

$$
\mathcal{L}=-\frac{1}{4} F_{\mu \nu}^{a} F_{a}^{\mu \nu}+\frac{1}{2} \Pi_{a b}^{\mu \nu} A_{\mu}^{a} A_{\nu}^{b}+\mathcal{L}_{(3)}^{1}+\mathcal{L}_{(4)}^{1}
$$

where $\mathcal{L}_{(3)}^{1}$ and $\mathcal{L}_{(4)}^{1}$ are the one loop lagrangian terms for the three and four point gluon vertices respectively. For the three point gluon vertex only the diagram in Fig. 2.2 survives at leading order in $1 / \mu$; for the four point gluon vertex only the diagram in Fig. 2.3 survives in the same limit.

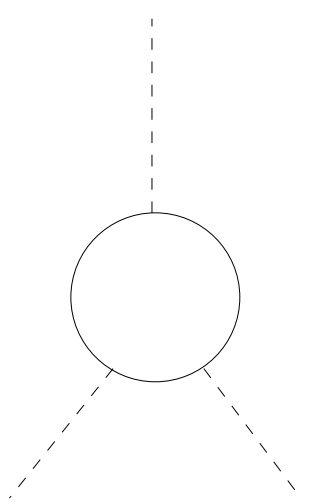

Fig. 2.2 Three gluon vertex. Dotted lines represent gluon fields; full lines are fermion propagators.

To start with, let us consider the three-point function. At the tree level $\left(\mathcal{L}=\mathcal{L}^{0}\right)$ the contribution to the lagrangian can be written in the form:

$$
\mathcal{L}_{(3)}^{0}=-g f_{a b c} A_{a}^{\mu} A_{b}^{\nu} \partial_{\mu} A_{c, \nu}
$$

At one loop $\left(\mathcal{L}=\mathcal{L}^{1}\right)$ we have to distinguish between the contribution of the diagram involving the gluons in the unbroken $S U(2)$ gauge group, which we call $\mathcal{L}_{(3), 1}^{1}$, and those involving gluons corresponding to broken generators, which we 


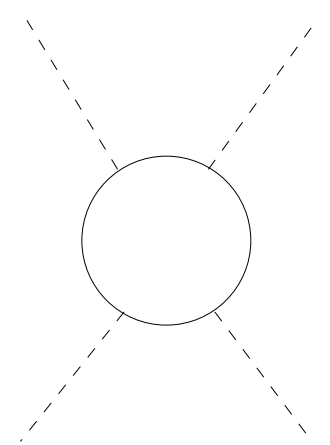

Fig. 2.3 Four gluon vertex. Dotted lines represent gluon fields; full lines are fermion propagators.

call $\mathcal{L}_{(3), 2}^{1}$. So the one-loop correction at the three gluon vertex may be written as follows:

$$
\mathcal{L}_{(3)}^{1}=\mathcal{L}_{(3), 1}^{1}+\mathcal{L}_{(3), 2}^{1} .
$$

For the $S U(2)$ contribution our result is as follows:

$$
\mathcal{L}_{(3), 1}^{1}=-g k f_{a b c} A_{a}^{\mu} A_{b}^{\nu} \partial^{\mu} A_{c}^{\nu}\left[\delta_{\mu 0} \delta_{\nu i}+\delta_{\mu i} \delta_{\nu 0}\right]
$$

with $a, b, c \in\{1,2,3\}$ and $k=\frac{g^{2} \mu^{2}}{18 \pi^{2} \Delta^{2}}$. This term is consistent with the fact that color superconductivity leaves invariant the subgroup $S U(2)_{c}$ generated by the $S U(3)_{c}$ generators $T^{a}, a=1,2,3$. It can be also obtained in a simpler manner, by requiring gauge invariance for the $S U(2)$ gluons. We do not write down explicitly $\mathcal{L}_{(3), 2}^{1}$ whose expression can be found in 31].

The same results are found for the four gluon vertex; again the structure of the vertex coincides with the one dictated by gauge invariance for the gluons 1,2 and 3 , while this invariance is broken for the other generators.

\subsection{Gap equation in HDET}

Let us conclude this section by deriving the gap equation in the High Density Effective Theory. Let us begin with the CFL case; to get the gap equation one can use the effective action formalism of Cornwall-Jackiw-Tomboulis [57]. In this context it corresponds to the calculation of the grand potential

$$
\Omega=\frac{1}{2} \frac{4 \pi \mu^{2}}{(2 \pi)^{4}} \int d^{2} \ell\left\{\operatorname{Tr} \ln \left[S_{\text {free }}^{-1}(\ell) S(\ell)\right]-\operatorname{Tr} S(\ell) \Sigma(\ell)\right\} .
$$

Here $S^{-1} \equiv S_{A B}^{-1}$ and $S_{\text {free }}^{-1} \equiv S_{A B}^{-1}$, free are respectively the inverse fermion propagator and the free inverse fermion propagator and $\Sigma=S^{-1}-S_{\text {free }}^{-1}$ is the proper self-energy. By extremization of the density energy with respect to the gap one gets the Schwinger-Dyson equations; the fermion equation is the desired gap equation. In our case the truncated Schwinger-Dyson equation assumes the form

$$
S_{A B}^{-1}(p=0)-S_{A B, \text { free }}^{-1}(p=0)=
$$




$$
=i \frac{4 \pi \mu^{2} g^{2}}{(2 \pi)^{4}} \int d^{2} \ell i D_{a b}^{\mu \nu} J_{\mu}^{a A C} i S_{C D}(\ell) J_{\mu}^{b D B} .
$$

Here

$$
J_{\mu}^{a A B}=g\left(\begin{array}{cc}
i V_{\mu} h_{A a B} & 0 \\
0 & -i \tilde{V}_{\mu} h_{A a B}^{*}
\end{array}\right)
$$

with $h_{A a B}$ given in (2.103). We assume a fictitious gluon propagator

$$
i D_{a b}^{\mu \nu}=i \frac{g^{\mu \nu} \delta^{a b}}{\Lambda^{2}},
$$

which corresponds to a local four fermion interactions similar to the NambuJona Lasinio model [2]. One obtains two equations:

$$
\begin{aligned}
\Delta & =-\frac{2 i \mu^{2} g^{2}}{4 \Lambda^{2} \pi^{3}}\left(\frac{\Delta_{9}}{6} I\left(\Delta_{9}\right)-\frac{\Delta}{3} I(\Delta)\right) \\
\Delta_{9} & =-\frac{2 i \mu^{2} g^{2} \Delta}{3 \Lambda^{2} \pi^{3}} I(\Delta)
\end{aligned}
$$

where

$$
I(\Delta)=\int \frac{d^{2} \ell}{V \cdot \ell \tilde{V} \cdot \ell-\Delta^{2}+i \epsilon}=-i 2 \pi \operatorname{arcsinh}\left(\frac{\delta}{|\Delta|}\right) .
$$

We fix $\Lambda / g$ by using the same equation and the same model for the gluon propagator at $\mu=0$, where the effect of the order parameter, in this case the chiral condensate, is to produce a constituent mass $M$ for the light quark. This assumption gives the equation

$$
1=\frac{4 g^{2}}{3 \Lambda^{2} \pi^{2}} \int_{0}^{K} \frac{p^{2}}{\sqrt{p^{2}+M^{2}}} .
$$

For $M=400 \mathrm{MeV}$ and the cutoff $K=800 \mathrm{MeV}(700 \mathrm{MeV})$ we get $\Lambda / g=181$ $\mathrm{MeV}(154 \mathrm{MeV})$. The corresponding values for the gap parameters are given in tables 2.2 and 2.3 .

\begin{tabular}{|c|c|c|c|}
\hline$K=\delta+\mu$ & $\Delta$ & $\Delta_{9}$ & $\Delta_{9} / \Delta$ \\
\hline $800 \mathrm{MeV}$ & $66 \mathrm{MeV}$ & $-150 \mathrm{MeV}$ & -2.27 \\
$700 \mathrm{MeV}$ & $77 \mathrm{MeV}$ & $-189 \mathrm{MeV}$ & -2.45 \\
\hline
\end{tabular}

Table $2.2 \Delta, \Delta_{9}$ and $\Delta_{9} / \Delta$ in the CFL model; $\mu=500 \mathrm{MeV}$.

\begin{tabular}{|c|c|c|c|}
\hline$K=\delta+\mu$ & $\Delta$ & $\Delta_{9}$ & $\Delta_{9} / \Delta$ \\
\hline $800 \mathrm{MeV}$ & $25 \mathrm{MeV}$ & $-57 \mathrm{MeV}$ & -2.28 \\
$700 \mathrm{MeV}$ & $51 \mathrm{MeV}$ & $-114 \mathrm{MeV}$ & -2.24 \\
\hline
\end{tabular}

Table 2.3 Same as in table 2.2, with $\mu=400 \mathrm{MeV}$.

In order to obtain these results we have used the same cutoff $K$ for the two gap equations at $\mu=0$ and $\mu \neq 0$; this fixes the value of $\delta$, the cutoff on the residual momentum along the Fermi velocity at $\mu \neq 0\left(\left|l_{\|}\right| \leq \delta\right)$ :

$$
\delta=K-\mu \text {. }
$$


A similar approach has been used by other authors, see for example the review [14] and references therein. Their calculation differs from the present one for two reasons: they include the $\mathcal{O}(1 / \mu)$ corrections to the gap equations and use a smooth cutoff

$$
F(p)=\left(\frac{\Lambda^{2}}{\Lambda^{2}+p^{2}}\right)^{\nu},
$$

with $\Lambda=800 \mathrm{MeV}$ and various values of $\nu$. They obtain at $\mu=400 \mathrm{MeV}$ $\Delta=80 \mathrm{MeV}$ and $\Delta_{9}=-176 \mathrm{MeV}$ while for $\mu=500 \mathrm{MeV} \Delta=109 \mathrm{MeV}$ and $\Delta_{9}=-249 \mathrm{MeV}$. Note that in any case the ratio $\Delta_{9} / \Delta \approx-2$ which means that the CFL ansatz is indeed justified by this analysis.

The analogous of eq. 2.183 for the 2SC case is

$$
1=\frac{i \mu^{2} g^{2}}{3 \Lambda^{2} \pi^{3}} I(\Delta)=\frac{2 \mu^{2} g^{2}}{3 \Lambda^{2} \pi^{2}} \operatorname{arcsinh}\left(\frac{\delta}{|\Delta|}\right)
$$

that can be solved explicitly:

$$
|\Delta|=\frac{\delta}{\sinh \left(\frac{3 \Lambda^{2} \pi^{2}}{2 \mu^{2} g^{2}}\right)} .
$$

With the same values of $\Lambda / g(\Lambda / g=181 \mathrm{MeV}$ and $154 \mathrm{MeV})$ we find the values for the gap parameter of the $2 \mathrm{SC}$ model reported in tables 2.4 and 2.5.

\begin{tabular}{|c|c|}
\hline$K=\delta+\mu$ & \multicolumn{1}{|c|}{$\Delta$} \\
\hline $800 \mathrm{MeV}$ & $88 \mathrm{MeV}$ \\
$700 \mathrm{MeV}$ & $105 \mathrm{MeV}$ \\
\hline
\end{tabular}

Table 2.4 Value of the parameter $\Delta$ in the $2 \mathrm{SC}$ model; $\mu=500 \mathrm{MeV}$.

\begin{tabular}{|c|c|}
\hline$K$ & $\Delta$ \\
\hline $800 \mathrm{MeV}$ & $39 \mathrm{MeV}$ \\
$700 \mathrm{MeV}$ & $68 \mathrm{MeV}$ \\
\hline
\end{tabular}

Table 2.5 Same as in Table 2.4 with $\mu=400 \mathrm{MeV}$.

\subsection{Appendix. A few useful integrals}

We list a few 2-D integrals used to obtain the results of this Section. Let us define

$$
I_{n}=\int \frac{d^{N} \ell}{\left(V \cdot \ell \tilde{V} \cdot \ell-\Delta^{2}+i \epsilon\right)^{n+1}}=\frac{i(-i)^{n+1} \pi^{\frac{N}{2}}}{n !} \frac{\Gamma\left(n+1-\frac{N}{2}\right)}{\Delta^{2 n+2-N}} ;
$$

therefore, for $N=2-\epsilon$ and with $\gamma$ the Euler-Mascheroni constant,

$$
\begin{aligned}
& I_{0}=-\frac{2 i \pi}{\epsilon}+i \pi \ln \pi \Delta^{2}+i \pi \gamma \\
& I_{1}=+\frac{i \pi}{\Delta^{2}}, I_{2}=-\frac{i \pi}{2 \Delta^{4}}, I_{3}=+\frac{i \pi}{3 \Delta^{6}} .
\end{aligned}
$$


Moreover we define

$$
I_{n, m}=\int \frac{d^{2} \ell}{\left(V \cdot \ell \tilde{V} \cdot \ell-\Delta^{2}+i \epsilon\right)^{n}\left(V \cdot \ell \tilde{V} \cdot \ell-\Delta^{\prime 2}+i \epsilon\right)^{m}} .
$$

We get

$$
\begin{aligned}
& I_{1,1}=\frac{i \pi}{\Delta^{2}-\Delta^{\prime 2}} \ln \frac{\Delta^{2}}{\Delta^{\prime 2}} \\
& I_{2,1}=i \pi\left[\frac{1}{\Delta^{2}\left(\Delta^{2}-\Delta^{\prime 2}\right)}-\frac{1}{\left(\Delta^{2}-\Delta^{\prime 2}\right)^{2}} \ln \frac{\Delta^{2}}{\Delta^{\prime 2}}\right] \\
& I_{3,1}=\frac{2 \pi}{2}\left[\frac{-1}{\Delta^{4}\left(\Delta^{2}-\Delta^{\prime 2}\right)}-\frac{2}{\Delta^{2}\left(\Delta^{2}-\Delta^{\prime 2}\right)^{2}}+\frac{2}{\left(\Delta^{2}-\Delta^{\prime 2}\right)^{3}} \ln \frac{\Delta^{2}}{\Delta^{\prime 2}}\right] \\
& I_{2,2}=i \pi\left[\left(\frac{1}{\Delta^{2}}+\frac{1}{\Delta^{\prime 2}}\right) \frac{1}{\left(\Delta^{2}-\Delta^{\prime 2}\right)^{2}}+\frac{2}{\left(\Delta^{2}-\Delta^{\prime 2}\right)^{3}} \ln \frac{\Delta^{2}}{\Delta^{\prime 2}}\right] .
\end{aligned}
$$

The following integral is used in the context of the 2SC model:

$$
\tilde{I}_{1}=\int \frac{(V \cdot \ell)^{2} d^{2} \ell}{(V \cdot \ell \tilde{V} \cdot \ell)^{2}}
$$

The divergence is cured going to imaginary frequencies and finite temperature $\left(\ell_{0}=i \omega_{n}, \omega_{n}=\pi T(2 n+1) ; T \rightarrow 0, \mu \rightarrow \infty\right)$ :

$$
\tilde{I}_{1}=2 \pi T i \int_{-\mu}^{+\mu} d x \sum_{-\infty}^{+\infty} \frac{\left(i \omega_{n}-x\right)^{2}}{\left(x^{2}+\omega_{n}^{2}\right)^{2}}=2 \pi T i\left(-\frac{1}{T} \tanh \frac{\mu}{2 T}\right) \rightarrow-2 \pi i .
$$

Other divergent integrals, such as

$$
\tilde{I}=\int \frac{d^{2} \ell}{(V \cdot \ell \tilde{V} \cdot \ell)\left(V \cdot \ell \tilde{V} \cdot \ell-\Delta^{2}\right)}
$$

are treated in a similar way.

Finally, useful angular integrations are:

$$
\begin{aligned}
\int \frac{d \vec{v}}{4 \pi} v^{j} v^{k} & =\frac{\delta^{j k}}{3} \\
\int \frac{d \vec{v}}{4 \pi} v^{i} v^{j} v^{k} v^{\ell} & =\frac{1}{15}\left(\delta^{i j} \delta^{k \ell}+\delta^{i k} \delta^{j \ell}+\delta^{i \ell} \delta^{j k}\right) .
\end{aligned}
$$




\section{Chapter 3}

\section{Theoretical developments}

\subsection{Advances in the CFL model}

In the last three years there has been a tremendous activity in the study of QCD at high density. To review these results is beyond the scope of this paper and I refer to some excellent review papers that have been already published [14]. I will survey here only some of the most important developments that in a way or in another are related to our subject, i.e. the effective lagrangian approach. To begin with, in this paragraph I report on some of these advances in the Color-Flavor-Locking model.

\subsubsection{Would-be NGBs masses}

In par. 1.5 the effective theory for the NGBs in the CFL was presented?. In the CFL model the Nambu Goldstone Bosons are the only elementary excitations that are massless in the limit of zero Dirac quark masses. To have a more realistic description one should however include the effect of Dirac quark masses, which implies that also the octet of would-be NGBs acquire mass (the superfluid mode remains massless). The Dirac masses induce a symmetry breaking term in the lagrangian that becomes:

$$
\mathcal{L}_{\text {eff }}=\frac{F^{2}}{4} \operatorname{Tr}\left(\partial_{t} \Sigma \partial_{t} \Sigma^{\dagger}-v^{2}|\vec{\nabla} \Sigma|^{2}\right)-c\left[\operatorname{det} M \operatorname{Tr}\left(M^{-1} \Sigma\right)+h . c .\right]
$$

where $M=\operatorname{diag}\left(m_{u}, m_{d}, m_{s}\right)$. The value of the parameter $c$ is 44, 47, 48, 49, 50]:

$$
c=\frac{3 \Delta^{2}}{2 \pi^{2}} \text {. }
$$

The masses of the different bosons in the octet depend linearly on the Dirac quark masses and have a pattern different from the analogous octet of pseudo NGBs of the chiral symmetry; for example one finds:

$$
m_{\pi^{ \pm}}^{2}=\frac{2 c}{F^{2}} m_{s}\left(m_{u}+m_{d}\right) . \quad m_{K^{ \pm}}^{2}=\frac{2 c}{F^{2}} m_{d}\left(m_{u}+m_{s}\right) .
$$

\footnotetext{
${ }^{1}$ In the $2 \mathrm{SC}$ model there is one NGB and three gluons remain massless. The effective theory for the 2SC model has been developed in 19]. Its coupling to electromagnetic field and weak currents is in 58 .
} 
Note that

$$
\frac{m_{K^{ \pm}}^{2}}{m_{\pi^{ \pm}}^{2}} \simeq \frac{m_{d}}{m_{u}+m_{d}},
$$

i.e. the kaon is lighter than the pion.

Numerically these masses are much smaller than the corresponding masses at zero densities, due to the suppression factor $\Delta / \mu$; for example the kaon mass, for $\mu \simeq 500 \mathrm{MeV}$ is in the range of $\sim 10 \mathrm{MeV}$.

\subsubsection{Electromagnetic interactions}

The diquark condensate breaks $U(1)_{e m}$, which is clear as it couples a quark pair with a non zero total charge. In presence of spontaneous breaking of electromagnetism photons acquire a Meissner mass and ordinary conductors become superconductors. In the case of color superconductors, however, the situation is different. Here a new $U(1)$ subgroup appears, arising from a combination of color and flavor; it remains unbroken and takes the role of electromagnetism in the high density phase, while the associated vector boson remains massless [3]. The situation is similar to the standard model of the electroweak interactions, where the photon arises as a combination of the two neutral bosons related to two diagonal generators of the $S U(2) \times U(1)$ electroweak group.

To determine the unbroken $U(1)$ group, let us show that the condensate

$$
\operatorname{Tr}\left[\psi^{T} \epsilon_{I} \psi \epsilon_{I}\right]
$$

see Eq. (2.33), is invariant under the $U(1)$ transformation

$$
\psi_{\alpha i} \rightarrow U_{i j}^{\alpha \beta} \psi_{\beta j}
$$

with

$$
U_{i j}^{\alpha \beta}=(\exp \{i \epsilon[\mathbf{1} \otimes Q+T \otimes \mathbf{1}]\})_{i j}^{\alpha \beta},
$$

where $Q=\operatorname{diag}(2 / 3,-1 / 3,-1 / 3)$ is the quark charge matrix in the flavor space and $T_{\alpha \beta}=1 / 3(-2,1,1)$ acts on color indices. In infinitesimal terms:

$$
\psi \rightarrow \psi+\delta \psi
$$

where

$$
\delta \psi_{\alpha i}=i \epsilon\left[\psi_{\alpha j} Q_{i j}+T_{\alpha \beta} \psi_{\beta j}\right],
$$

To prove the invariance under the $U(1)$ group generated by

$$
\tilde{Q}=\mathbf{1} \otimes Q+T \otimes \mathbf{1}
$$

it is sufficient to note that

$$
\delta \operatorname{Tr}\left[\psi^{T} \epsilon_{I} \psi \epsilon_{I}\right]=2 i \epsilon\{[\operatorname{Tr}(\psi T \psi+\psi \psi Q)-\operatorname{Tr}(\psi) \operatorname{Tr}(T \psi+\psi Q)]\}=0,
$$

where we have used (2.35). Therefore the unbroken electromagnetism arises from the combination of the original $U(1)_{e m}$ with the $U(1)$ subgroup generated by a color hypercharge. 
Let us now consider the vector boson taking the place of the photon. It would appear in the lagrangian through the covariant derivative

$$
D_{\mu}=\partial_{\mu}-i\left(e A_{\mu} Q+g G_{\mu}^{8} \sqrt{3} T\right)
$$

$\left(T^{8}=\sqrt{3} T\right)$. Writing

$$
\begin{aligned}
A_{\mu} & =\cos \theta \tilde{A}_{\mu}-\sin \theta \tilde{G}_{\mu}^{8}, \\
G_{\mu}^{8} & =+\sin \theta \tilde{A}_{\mu}+\cos \theta \tilde{G}_{\mu}^{8},
\end{aligned}
$$

one can see that the field coupled with a strength proportional to $\tilde{Q}$ is $\tilde{A}_{\mu}$, obtained from these equations with

$$
\cos \theta=\frac{g}{\sqrt{g^{2}+\frac{e^{2}}{3}}} .
$$

Therefore the field

$$
\tilde{A}_{\mu}=\cos \theta A_{\mu}+\sin \theta G_{\mu}^{8}
$$

takes the role of the massless photon field, while

$$
\tilde{G}_{\mu}^{8}=-\sin \theta A_{\mu}+\cos \theta G_{\mu}^{8}
$$

is a massive gluon. We note that, since $g \gg e$, the angle $\theta$ is small, $\theta \approx 0$ and the effective photon is mostly made by the ordinary photon. We also note that the electromagnetic charges $\tilde{Q}$ of the quarks are produced by combining two sets of charges, the charges $q_{i}=(2 / 3,-1 / 3,-1 / 3)$ and the charges $t_{\alpha}=1 / 3(-2,1,1)$; the quarks have therefore always integer electromagnetic charges, as in the old Han-Nambu model. The unit of the electric charge, corresponding to the absolute value of the electron charge, is 2

$$
\tilde{e}=\frac{e g}{\sqrt{g^{2}+\frac{e^{2}}{3}}} .
$$

The quarks up with colors $r, g, b$ have respectively charges $0,+\tilde{e},+\tilde{e}$; the quarks down (or strange) with colors $1,2,3$ have respectively charges $-\tilde{e}, 0,0$. It is worth noticing that also the gluons have integer electric charge $\tilde{Q}$, exactly as the octet of vector bosons of low density QCD. The discussion of the electroweak couplings in the context of the effective theories can be found in [58].

\subsubsection{Quark-hadron continuity}

Let us summarize once again the properties of the low energy spectrum in the CFL model. Besides the octet+singlet quasi-particles of spin $\frac{1}{2}$ with integer charge there is an octet of massive vector states, again with integer charges and an octet of pseudoscalar NGBs: they are massless in the limit of zero quark

\footnotetext{
${ }^{2}$ The electron charge is less than $e$ because the electron couples to the photon with a strength reduced by $\cos \theta$.
} 
masses, but acquire masses if the chiral symmetry is explicitly broken; finally there is a massless NGB associated with the breaking of $U(1)_{B}$ that remains massless even in presence of quark masses. There is also a light pseudoscalar bosons that is never massless: it is associated to the symmetry $U(1)_{A}$ which is broken by instantons. If we now compare these results with the symmetries of the 3-flavor hypernuclear matter [59] we see a considerable similarity. On one side we have the integer-charge low lying baryonic octet $N, \Sigma, \Lambda$ whose members can pair to produce $S U(3)$ condensates, e.g. $\Lambda \Lambda, \Sigma \Sigma$ etc. breaking the baryonic number; on the other side there are the octet+singlet quasiparticles of spin $1 / 2$ with integer charges as well. On the nuclear side we have the low mass pseudoscalar octet of pions, kaons etc. and on the other side the octet of NGB of the broken symmetry of the CFL phase. Finally to the low mass $J^{P}=1^{-}$octet of vector resonances $\left(\rho, K^{*}\right.$, etc.) would correspond the massive gluons. All that means that probably the two phases are contiguous and one passes from one to the other without phase transition. A further example will be discussed in paragraph 3.2.2.

\subsubsection{Asymptotic analysis}

We have derived in the paragraph 2.6 the gap equation in the Nambu-Jona Lasinio model where the gluon propagator is substituted by a local four-fermion coupling. Eq. (2.183) has the approximate solution in the CFL model

$$
\Delta \approx 2 \delta \exp \left\{-\frac{3 \Lambda^{2} \pi^{2}}{\mu^{2} g^{2}}\right\}
$$

A more refined treatment would involve the use of the gluon propagator and has been performed in [60], [61] (see also [62] and Barrois and Frautschi in [1]). The result of this analysis is given by the formula:

$$
\Delta \approx \frac{\mu}{g^{5}} \exp \left\{-\frac{3 \pi^{2}}{\sqrt{2} g}\right\} .
$$

One should note the different behavior

$$
\Delta \sim \exp (- \text { const } / g)
$$

instead of $\Delta \sim \exp \left(-\right.$ const $\left./ g^{2}\right)$ between (3.20) and (3.19), which is due to the fact that in the complete treatment the gluon propagator at high density has a collinear divergence at zero scattering angle. We can qualitatively understand the behavior (3.21) as follows [14]. The collinear divergence should be regulated by the gluon rest mass that, as we know, is of the order $\Delta$, see par. 2.4.2. Therefore the gap equation has the form

$$
\Delta \propto g^{2} \mu^{2} \int d \ell_{0} d \theta \frac{\Delta}{\sqrt{\ell_{0}^{2}+\Delta^{2}}} \frac{1}{\mu^{2} \theta+\Delta^{2}} .
$$

In this way one gets the relation

$$
1 \propto g^{2}\left(\ln \frac{\Delta}{\mu}\right)^{2}
$$

and therefore eq. (3.21). 


\subsection{Other models}

\subsubsection{One flavor}

In the real world $m_{u} \simeq m_{d} \simeq 0$ might be a good approximation; therefore, if

$$
\Delta>m_{s}^{2} /(2 \mu)
$$

the color flavor locking could be a good approximation as well. The reason for the condition (3.24) can be understood as follows (see [38] and the discussion on the strange quark mass in [⿶] and [7]). The main effect of the strange quark mass is kinematic and produces a shifting between the Fermi momenta of the $u, d$ quarks, $p_{F} \simeq \mu$, and that of the strange quark, $p_{F}=\sqrt{\mu^{2}-m_{s}^{2}} \simeq \mu-\frac{m_{s}^{2}}{2 \mu}$. The difference between the two momenta must be small for the pairing to occur, in particular it must be smaller than the smearing of the Fermi surface, of order $\Delta$, which explains (3.24).

On the other hand, if $m_{s}>\sqrt{2 \mu \Delta}$, one has to consider the role played by the strange quark explicitly. Quantum Cromo Dynamics at large chemical potential and small $T$ with one flavor (strangeness) has been considered in 43]. Also in this case the theory exhibits color superconductivity, however the symmetry breaking condensate, being antisymmetric in color, must be in an angular momentum 1 state, because there is no other way to obtain an antisymmetric wavefunction for the strange quark pair. In the non relativistic limit the computations are relatively simple 3 ; there are two possibilities for the order parameter $\Delta_{i}^{a}$, i.e.

$$
\left\langle\psi^{T} C \sigma^{i} \hat{\lambda}_{A}^{a} \psi\right\rangle, \quad\left\langle\psi^{T} C \hat{q}^{i} \hat{\lambda}_{A}^{a} \psi\right\rangle
$$

here $\hat{\lambda}_{A}^{a}(a=1,2,3)$ are the three Gell-Mann matrices antisymmetric in color (i.e. $\left.\lambda_{2}, \lambda_{5}, \lambda_{7}\right)$; the two terms in (3.25) are distinct because in the non relativistic limit spin and orbital angular momentum are separately conserved. Four possible cases were considered [43]:

$$
\begin{aligned}
\Delta_{i}^{a} & =\Delta \delta_{i}^{a}, \\
\Delta_{i}^{a} & =\Delta \delta^{a 3} \delta_{i 3}, \\
\Delta_{i}^{a} & =\Delta \delta^{a 3}\left(\delta_{i 1}+i \delta_{i 2}\right), \\
\Delta_{i}^{a} & =\Delta\left(\delta^{a 1} \delta_{i 1}+\delta^{a 2} \delta_{i 2}\right),
\end{aligned}
$$

called respectively Color Spin Locking (CSL), 2SC, A and planar phase. For the former condensate in (3.25) 1 the results are as follows. First one determines the value of the parameter $\Delta$ solving the Schwinger-Dyson equation for the four cases in (3.26); one obtains that the largest gap corresponds to the CSL case. Next one looks at the grand potential and observes that the solution minimizing it corresponds again to the Color Spin Locking phase. One can conclude that the $C S L$ phase is indeed favored for the case of one-flavor QCD at high density.

\footnotetext{
${ }^{3}$ The analysis of the ultrarelativistic limit does not change the conclusions obtained by the non relativistic approximation.

${ }^{4}$ The latter condensate produces either smaller or anisotropic gaps and the analysis is more involved, see 43 for further discussion.
} 
The global symmetries of the $C S L$ phase agree with the expectations based on $N_{f}=1 \mathrm{QCD}$ at high density, and therefore one can argue that also in this case, as for CFL model with $N_{f}=3$, low and high density phases are continuously connected.

\subsubsection{QCD at large isospin density}

It would be desirable to confront the analytical results obtained by different approximations for QCD at high density with the lattice QCD calculations. However this comparison is at present impossible because current methods to evaluate the QCD partition function use path integral with a complex fermion determinant. In the quenched QCD approximation one ignores the fermionic determinant, but this procedure leads to inconsistent results in the case of finite baryonic chemical potential $\mu_{B}$ [63], [64]. In absence of a consistent approximation scheme, one may hope to get a clue to the behaviour of quark matter at finite $\mu_{B}$ by considering QCD at finite $\mu_{I}$, where $\mu_{I}$ is the chemical potential associated to the third component of isospin, $I_{3}$. In 65 the case of finite $\mu_{I}$ and $\mu_{B}=0$ was considered, which, though unrealistic, can be of some utility because it should be accessible to lattice calculations [66]. The results found in 65] are as follows. When $\left|\mu_{I}\right|<m_{\pi}$ the vacuum does not differ from the $\mu_{I}=0$ case, because to excite a $\pi$ costs $m_{\pi}-\left|\mu_{I}\right|$. If $\left|\mu_{I}\right|$ is small, but larger than $m_{\pi}$, the excitation of the pions is favored and the ground state is a pion superfluid (with one massless pion and the other two massive). This state is characterized by the order parameter $\left\langle\pi^{-}>\neq 0\right.$. For large $\left|\mu_{I}\right|$ one goes to a region characterized by the order parameter $\left\langle\bar{u} \gamma_{5} d>\neq 0\right.$. Since the two order parameters $\left\langle\pi^{-}>\neq 0\right.$ and $\left\langle\bar{u} \gamma_{5} d\right\rangle \neq 0$ have the same quantum numbers and break the same symmetries it can be conjectured that there is no phase transition along the $\mu_{I}$ axis from the small to the high chemical potential 65] and one can move continuously from the hadron to the quark phase. This hypothesis is similar to the conjecture of quark hadron continuity discussed in the CFL model, see paragraph 3.1 .3 .

\subsection{Transport theory for quarks in dense matter}

The transport theory for colored particles in a Yang Mills external field can be used to derive quasi-particle properties for QCD at high density. This development not only allows a different derivation of known results, but also sheds a new light on the dynamics of the quasi-particles.

The classical equations describing the motion of a particle in colored external field have been derived by Wong [67]. Here we derive the Wong equations by the Fok Schwinger proper time method that presents the advantage of being covariant.

To begin with, we write the Dirac equation for the Feynman propagator

$$
\left(i D_{x}-m\right) S_{F}\left(x, x^{\prime}\right)=\delta^{4}\left(x-x^{\prime}\right)
$$

\footnotetext{
${ }^{5}$ The original Wong derivation used Dirac equation. The quadratic form of Dirac equation was used by Heinz 68 to disentangle quark spin.
} 
in an operatorial way

$$
(i \not D-m) S_{F}=1 \text {. }
$$

We have

$$
S_{F}=\frac{1}{i \not D-m}=\frac{i \not D+m}{-2 m} \frac{-2 m}{(i \not D)^{2}-m^{2}}=\frac{i \not D+m}{-2 m} G,
$$

with

$$
G=\frac{-2 m}{(i \not D)^{2}-m^{2}} \equiv \frac{1}{H} .
$$

Let us interpret $H$, given by

$$
H=\frac{(i \not D)^{2}-m^{2}}{-2 m},
$$

as a hamiltonian; with $\tau$ proper time of the quark we have:

$$
H \Psi=i \frac{\partial \Psi}{\partial \tau},
$$

i.e.

$$
i \frac{\partial U}{\partial \tau}=H U
$$

where

$$
U=U(\tau)=e^{-i H \tau}
$$

is the propagator. In the coordinate representation one has

$$
U\left(x, x^{\prime}, \tau\right)=<x|U(\tau)| x^{\prime}>.
$$

Since one has

$$
G=\frac{1}{H}=-i \int_{-\infty}^{0} d \tau e^{-i H \tau}
$$

we also have

$$
\begin{aligned}
i \partial_{\tau}<x|U(\tau)| x^{\prime}> & =<x|H U| x^{\prime}>=<x\left|U U^{\dagger} H U\right| x^{\prime}>= \\
& =<x(\tau)|H(x(\tau), \pi(\tau), Q(\tau))| x^{\prime}(0)>
\end{aligned}
$$

where

$$
\pi^{\mu}=i D^{\mu}=p^{\mu}+g A^{\mu}
$$

is the kinetic momentum, and

$$
\begin{aligned}
\mid x(\tau)> & =U^{\dagger}(\tau) \mid x(0)>, \\
\mid x> & =\mid x(0)>.
\end{aligned}
$$

Moreover

$$
U^{\dagger}(\tau) H U(\tau)=H(x(\tau), \pi(\tau), Q(\tau))
$$

is the hamiltonian and

$$
\pi(\tau)=U^{\dagger}(\tau) \pi U(\tau)
$$




$$
\begin{aligned}
x(\tau) & =U^{\dagger}(\tau) x U(\tau), \\
Q_{a}(\tau) & =U^{\dagger}(\tau) Q_{a} U(\tau)
\end{aligned}
$$

are operators in the Heisenberg representation.

The Heisenberg equations of motion are as follows:

$$
\begin{aligned}
\frac{d x^{\mu}}{d \tau} & =i\left[H, x^{\mu}\right], \\
\frac{d \pi^{\mu}}{d \tau} & =i\left[H, \pi^{\mu}\right], \\
\frac{d Q_{a}}{d \tau} & =i\left[H, Q_{a}\right] .
\end{aligned}
$$

Since the following expression holds for $H$ :

$$
\begin{aligned}
H & =\frac{-1}{2 m}(\not t-m)(\not t+m)= \\
& =\frac{-1}{2 m}\left[\left(p^{\mu}+g \hat{A}^{\mu}\right)^{2}+\frac{g}{2} \sigma \cdot \hat{F}-m^{2}\right],
\end{aligned}
$$

the Heisenberg equations are transformed as follows. The first equation in (3.32) becomes

$$
\frac{d x^{\mu}}{d \tau}=\frac{\pi^{\mu}}{m}
$$

as expected. The analogous of the Lorentz force is as follows:

$$
\frac{d \pi^{\mu}}{d \tau}=\frac{g}{2 m}\left(\pi_{\lambda} \hat{F}^{\lambda \mu}+\hat{F}^{\lambda \mu} \pi_{\lambda}-\frac{1}{2} D^{\mu}(\sigma \cdot \hat{F})\right),
$$

while the dynamics of the color charge is governed by the equation

$$
\frac{d Q_{a}}{d \tau}=\frac{-g}{2 m} f_{a b c}\left(\pi_{\lambda} A_{b}^{\lambda} Q^{c}+A_{b}^{\lambda} Q^{c} \pi_{\lambda}+\frac{1}{2}\left(\sigma \cdot F^{b}\right) Q^{c}\right) .
$$

Since in the classical limit all the operators commute, one has

$$
\begin{aligned}
{[\pi, F] } & =0, \\
{\left[Q^{a}, Q^{b}\right] } & =i \hbar f_{a b c} Q^{c} \rightarrow 0 .
\end{aligned}
$$

The spin terms can also be neglected [68] and one obtains the Wong equations 67] $\left(\hat{F}^{\lambda \mu}=-Q^{a} F^{a \lambda \mu}\right.$ and $Q^{a}$ analogous of $-\lambda / 2$ in the classical limit [68]):

$$
\begin{aligned}
& m \frac{d x^{\mu}}{d \tau}=\pi^{\mu} \\
& m \frac{d \pi^{\mu}}{d \tau}=g Q^{a} F^{a \mu \lambda} \pi_{\lambda}, \\
& m \frac{d Q_{a}}{d \tau}=-g f_{a b c} \pi_{\lambda} A^{b \lambda} Q^{c} .
\end{aligned}
$$


Let us now consider the classical transport equation for the one particle distribution function $f(x, p, Q)$ which is the probability for finding the particle in the state $(x, p, Q)$ :

$$
m \frac{d f(x, p, Q)}{d \tau}=C[f](x, p, Q)
$$

where $C[f]$ denotes the collision integral. Putting

$$
D_{\mu}=\partial_{\mu}-g f_{a b c} Q^{c} A_{\mu}^{b} \partial_{a}^{Q}
$$

from equations (3.35) in the approximation $C[f]=0$ we get therefore the Boltzmann equation in the collisionless case:

$$
\left[D_{t}+\vec{v} \cdot \vec{D}-g Q^{a}\left(\vec{E}^{a}+\vec{v} \wedge \vec{B}^{a}\right) \frac{\partial}{\partial \vec{p}}\right] f=0 .
$$

Notice that in (3.38) the velocity

$$
\vec{v} \equiv \frac{\partial \epsilon(\vec{p})}{\partial \vec{p}}
$$

is determined by the dispersion law of the quasiparticle.

Eq. (3.38), together with the Yang Mills equations, that we do not write here, represent the complete set of Boltzmann-Vlasov equations for the non abelian case and have been considered in 69] to derive the hard thermal loops of QCD. In [70] Litim and Manuel apply it to the two-flavor color superconductor. The method can be summarized as follows. First of all one writes the colored current associated to the motion of the quasi-particle:

$$
J_{\mu}^{a}(x)=g \sum \int \frac{d^{3} p}{(2 \pi \hbar)^{3}} d Q v_{\mu} Q^{a} f(x, p, Q),
$$

where the sum is over the helicities and $v^{\mu}=(1, \vec{v})$. Let us call

$$
f^{e q}=\frac{1}{1+e^{\beta \epsilon}}
$$

the Fermi equilibrium distribution function and let us assume a small, perturbative deviation from equilibrium:

$$
f=f^{e q}+g \delta f .
$$

One also defines the color density

$$
J^{a}(x, p)=g \int d Q Q^{a} f(x, p, Q),
$$

so that (3.40) can be written as follows:

$$
J_{\mu}^{a}(x)=\int \frac{d^{3} p}{(2 \pi \hbar)^{3}} v_{\mu} J^{a}(x, p) .
$$


Expanding (3.38) in $g$ one obtains the transport equation for the color density:

$$
\left[D_{t}+\vec{v} \cdot \vec{D}\right] J(x, p)=g^{2} N_{f} \vec{v} \cdot \vec{E} \frac{d f^{e q}}{d \epsilon} .
$$

The formal solution of 3.42 is

$$
J_{\mu}^{a}=g^{2} N_{f} \int \frac{d^{3} p d^{4} y}{(2 \pi)^{3}} v_{\mu} G^{a b} \vec{v} \cdot \vec{E}^{b}(y) \frac{d f^{e q}}{d \epsilon},
$$

where $G^{a b}=<y\left|(v \cdot D)^{-1}\right| x>_{a b}$ is the Green function. We observe that, since $D$ depends on the gluon fields $A, J_{\mu}^{a}$ is a functional of $A$. This treatment is analogous to the derivation of the Hard Thermal Loop and the Hard Dense Loop approximations from the transport theory [69, [71], [72]. For the non relativistic and Abelian case it reduces to the transport theory for the BCS superconductor [73].

All the relevant information is contained in the functional $J[A]$ because the effective action $\Gamma$ can be obtained from $J[A]=-\frac{\delta \Gamma}{\delta A}$ and all the diagrams can be obtained by deriving $\Gamma$. As a matter of fact, expanding $J[A]$ in powers of $A_{\mu}$ one gets

$$
J_{\mu}^{a}[A]=\Pi_{\mu \nu}^{a b} A_{b}^{\nu}+\frac{1}{2} \Gamma_{\mu \nu \lambda}^{a b c} A_{b}^{\nu} A_{c}^{\lambda} .
$$

Therefore from (3.43) one can obtain the various tensors, in particular the polarization tensor $\Pi_{\mu \nu}^{a b}$, that in [70] is computed for the 2SC model.

There are several advantages in computing the polarization tensor by this method. First of all one obtains at once not only the results at $T=0$, but also the thermal dependence. For example the $T$-dependence of the Debye mass is given by

$$
m_{D}^{2}=-\frac{g^{2} N_{f}}{2 \pi^{2}} \int_{0}^{\infty} d p p^{2} \frac{d f^{e q}}{d \epsilon} \equiv \frac{g^{2} \mu^{2} N_{f}}{2 \pi^{2}} \hat{I}_{0}\left(\frac{\Delta}{T}, \frac{T}{\mu}\right)
$$

where

$$
\hat{I}_{n}\left(\frac{\Delta}{T}, \frac{T}{\mu}\right)=-\frac{1}{\mu^{2}} \int_{0}^{\infty} d p p^{2} v^{n} \frac{d f^{e q}}{d \epsilon} .
$$

One can check that $I_{n}(\infty, 0)=0$, which implies that there is no Debye screening in the $2 \mathrm{SC}$ model at $T=0$, a result we already knew. For small temperatures one gets

$$
m_{D}^{2} \sim \frac{g^{2} \mu^{2} N_{f}}{2 \pi^{2}} \sqrt{\frac{2 \pi \Delta}{T}} e^{-\Delta / T} .
$$

From the real part of $\Pi_{\mu \nu}^{a b}$ one can get the dispersion laws; the resulting expressions are involved, but can be expanded for small momenta, similarly to the approximations presented in the par. 2.4.2. On the other hand the imaginary part of $\Pi_{\mu \nu}^{a b}$ gives the Landau damping, that, however, is absent for small energies, i.e. plasmon and transverse excitations are stable provided $E_{L, T}(k)>k$. In [70] a comparison with the results obtained by quantum field theory at $T \neq 0$ [54 is given and an agreement is found. The method based on the transport equation is however more powerful because it allows the calculation of other 
significant quantities beyond the leading order, such as, for example, the damping rate, or transport properties such as thermal and electrical conductivities or shear viscosity that are dominated by the excitations of the NGB or the gapless quarks. 


\section{Chapter 4}

\section{The LOFF phase}

\subsection{Overview of the crystalline superconductive (LOFF) state}

In nature flavor symmetry is broken not only explicitly by quark mass terms, but also by weak interactions. Therefore in the applications of the color superconductivity one has to take into account this symmetry breaking; for example in compact stars, considering only two flavors, isospin is broken by $\delta \mu=\mu_{u}-\mu_{d} \neq 0$, due to the process:

$$
d \rightarrow \text { uev }
$$

When the two quarks in the Cooper pair have different chemical potentials, the vacuum is characterized, for certain values of $\delta \mu$, by a non vanishing expectation value of a quark bilinear breaking translational and rotational invariance. The appearance of this condensate is a consequence of the fact that in a given range of $\delta \mu$ [35], the formation of a Cooper pair with a total momentum

$$
\vec{p}_{1}+\vec{p}_{2}=2 \vec{q} \neq \overrightarrow{0}
$$

is energetically favored in comparison to the BCS state characterized by $\vec{q}=$ $\vec{k}+(-\vec{k})=0$. For the two flavor case one finds 35

$$
\begin{aligned}
\delta \mu & \in\left[\delta \mu_{1}, \delta \mu_{2}\right], \\
\delta \mu_{1} & =0.71 \Delta, \quad \delta \mu_{2}=0.74 \Delta, \delta \mu_{j} \ll \mu
\end{aligned}
$$

where $\Delta$ is the BCS gap. A similar phenomenon was already observed many years ago in the context of the BCS theory for superconducting materials in presence of magnetic impurities by Larkin, Ovchinnikov, Fulde and Ferrel and the corresponding phase is named LOFF state 40.

The exact form of the order parameter (diquark condensate) breaking spacetime symmetries in the crystalline phase is not yet known. In 35 the following ansatz is made:

$$
\Delta(\vec{x})=\Delta e^{i 2 \vec{q} \cdot \vec{x}}=\Delta e^{i 2 q \vec{n} \cdot \vec{x}}
$$




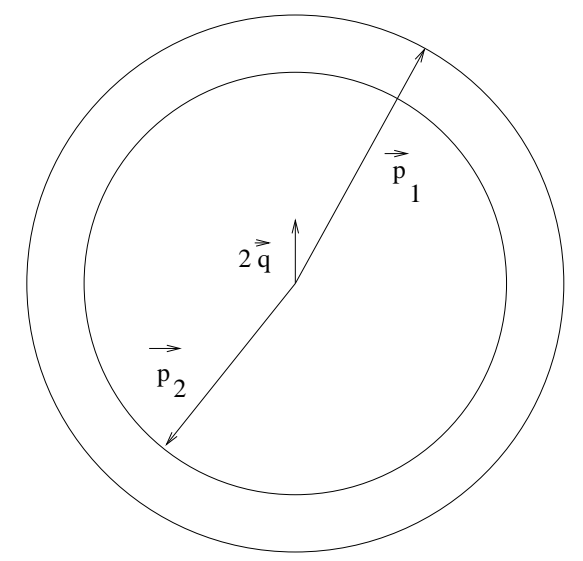

Fig. 4.1 LOFF kinematics. The Cooper pair has a total momentum $2 \vec{q} \neq 0$.

The value of $|\vec{q}|$ is fixed by the dynamics, while its direction $\vec{n}$ is spontaneously chosen. In the sequel I will assume the ansatz (4.4) as well. The order parameter (4.4) induces a lattice structure given by parallel planes perpendicular to $\vec{n}$ :

$$
\vec{n} \cdot \vec{x}=\frac{\pi k}{q} \quad(k=0, \pm 1, \pm 2, \ldots) .
$$

We can give the following physical picture of the lattice structure of the LOFF phase: Due to the interaction with the medium, the Majorana masses of the red and green up and down quarks have a periodic modulation in space, reaching on subsequent planes maxima and minima.

The Cooper pair can be formed only if quarks are in the antisymmetric channel, where there is a color attractive interaction; therefore it must be in antisymmetric flavor state if it in antisymmetric spin state $(S=0)$. The condensate has therefore the form

$$
-<0\left|\epsilon_{i j} \epsilon_{\alpha \beta 3} \psi^{i \alpha}(\vec{x}) C \psi^{j \beta}(\vec{x})\right| 0>=2 \Gamma_{A}^{L} e^{2 i \vec{q} \cdot \vec{x}} .
$$

The analysis of [35] shows that, besides the condensate (4.6) (scalar condensate), another different condensate is possible, i.e. one characterized by total spin 1 (vector condensate) and by a symmetric flavor state:

$$
i<0\left|\sigma_{i j}^{1} \epsilon_{\alpha \beta 3} \psi^{i \alpha}(\vec{r}) C \sigma^{03} \psi^{j \beta}(\vec{x})\right| 0>=2 \Gamma_{B}^{L} e^{2 i \vec{q} \cdot \vec{x}} .
$$

Note that in the BCS state the quarks forming the Cooper pair have necessarily $S=0$.

It goes without saying that the hypotheses of [35] are rather restrictive, as these authors assume only two flavors and make the ansatz of a plane wave behavior, Eq. (4.4). In any event their results are as follows. Assuming a point-like interaction as the origin of the Fermi surface instability, the LOFF state is energetically favored in the above-mentioned small range of values of $\delta \mu$ around $\delta \mu \sim 0.7 \Delta$. The actual value of the window range compatible with the presence of the LOFF state depends on the calculation by which the crystalline color state is computed. While the determination of the interval (4.3) is based 
on a local interaction, assuming gluon exchange, as in [36], the window opens up considerably. The value of the gap parameters will be discussed below, at the end of par. 4.3 .

The order parameters (4.6) and (4.7) spontaneously break rotational and translational symmetries. Associated with this breaking there will be Nambu Goldstone Bosons as in a crystal; these quasi-particles are known as phonons. Let us discuss them in some detail.

\subsection{Phonons in the LOFF phase}

For a generic lattice structure it is known that there are three phonons associated to the breaking of space symmetries. However one can show [32] that in order to describe the spontaneous breaking of space symmetries induced by the condensates (4.6) and (4.7) one NGB is sufficient. The argument (see Fig. 4.2) is as follows. Rotations and translations are not independent transformations, because the result of a translation plus a rotation, at least locally, can be made equivalent to a pure translation. Let us discuss the consequence of this fact and consider the goldstone field associated to the spontaneous breaking of the rotational and translational invariance. In the present case the NGB is a long

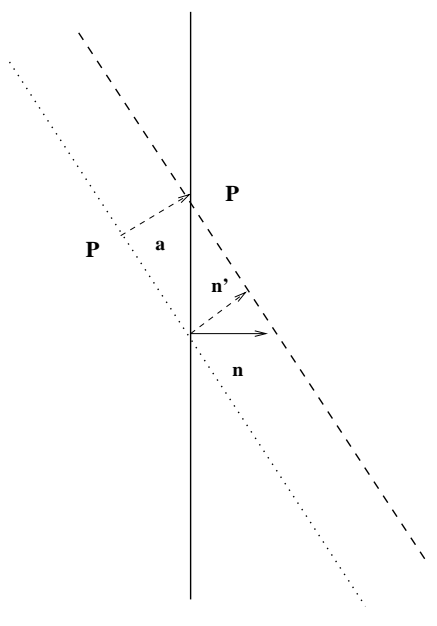

Fig. 4.2 In the point $\mathrm{P}$ the effect of the rotation $\vec{n} \rightarrow \vec{n}^{\prime}$ and the effect of the translation $\vec{x} \rightarrow \vec{x}+\vec{a}$ tend to compensate each other.

wavelength small amplitude variation of the condensate $\Delta(\vec{x}) \rightarrow \Delta(\vec{x}) e^{i \phi / f}$, with

$$
\phi / f=2 q(\vec{n}+\delta \vec{n}) \cdot(\vec{x}+\delta \vec{x})-2 q \vec{n} \cdot \vec{x}=2 q \vec{R} \cdot \vec{x}+T-2 q \vec{n} \cdot \vec{x},
$$

where we have introduced the auxiliary function $T$, given by $T=2 q \vec{R} \cdot \delta \vec{x}$.

Now the lattice fluctuations $\phi / f$ must be small; from this it follows that $T$ and $\vec{R}$ are not independent fields and therefore $T$ must depend functionally on

\footnotetext{
${ }^{1} \mathrm{~A}$ similar argument is used in [74] in a different context.
} 
$\vec{R}$, i.e. $T=F[\vec{R}]$, which, using again 4.8 , means that

$$
\Phi \equiv 2 q \vec{n} \cdot \vec{x}+\phi / f=2 q \vec{R} \cdot \vec{x}+F[\vec{R}] \equiv G[\vec{R}, \vec{x}] .
$$

The solution of this functional relation has the form

$$
\vec{R}=\vec{h}[\Phi]
$$

where $\vec{h}$ is a vector built out of the scalar function $\Phi$. By this function one can only円 form the vector $\vec{\nabla} \Phi$; therefore we get

$$
\vec{R}=\frac{\vec{\nabla} \Phi}{|\vec{\nabla} \Phi|}
$$

which satisfies $|\vec{R}|=1$ and $<\vec{R}>_{0}=\vec{n}$. In terms of the phonon field $\phi$ the field $\vec{R}$ is given at the first order in $\phi$ by the expression

$$
\vec{R}=\vec{n}+\frac{1}{2 f q}[\vec{\nabla} \phi-\vec{n}(\vec{n} \cdot \vec{\nabla} \phi)] .
$$

In order to derive the form of the interaction between the NGB and the quarks let us now write the effective lagrangian in the LOFF phase.

\subsection{HDET approach to the LOFF state}

To take into account the two non vanishing vacuum expectation values we add the term:

$$
\begin{aligned}
\mathcal{L}_{\Delta} & =\mathcal{L}_{\Delta}^{(s)}+\mathcal{L}_{\Delta}^{(v)}= \\
& =-\frac{e^{2 i \vec{q} \cdot \vec{x}}}{2} \epsilon^{\alpha \beta 3} \psi_{i \alpha}^{T}(x) C\left(\Delta^{(s)} \epsilon_{i j}+\vec{\alpha} \cdot \vec{n} \Delta^{(v)} \sigma_{i j}^{1}\right) \psi_{i \beta}(x) \\
& + \text { h.c. }
\end{aligned}
$$

to the lagrangian.

Let us first consider the scalar condensate:

$$
\mathcal{L}_{\Delta}^{(s)}=-\frac{\Delta^{(s)}}{2} e^{2 i \vec{q} \cdot \vec{x}} \epsilon^{\alpha \beta 3} \epsilon_{i j} \psi_{i \alpha}^{T}(x) C \psi_{i \beta}(x)-(L \rightarrow R)+\text { h.c. } .
$$

Here $\psi(x)$ are positive energy left-handed fermion fields discussed in the previous section. We neglect the negative energy states, consistently with the assumptions of HDET.

In order to introduce velocity dependent positive energy fields

$$
\psi_{+, \vec{v}_{i} ; i \alpha}
$$

\footnotetext{
${ }^{2}$ In principle there is a second vector, $\vec{x}$, on which $\vec{R}$ could depend linearly, but this possibility is excluded because $\vec{R}$ is a vector field transforming under translations as $\vec{R}(\vec{x}) \rightarrow$ $\vec{R}^{\prime}\left(\vec{x}^{\prime}\right)=\vec{R}(\vec{x})$.
} 
with flavor $i$, we have to decompose the fermion momenta:

$$
\vec{p}_{j}=\mu_{j} \vec{v}_{j}+\vec{\ell}_{j} \quad(j=1,2) .
$$

Therefore we have :

$$
\begin{aligned}
\mathcal{L}_{\Delta}^{(s)} & =-\frac{\Delta^{(s)}}{2} \sum_{\vec{v}_{1}, \vec{v}_{2}} \exp \left\{i \vec{x} \cdot \vec{\alpha}\left(\vec{v}_{1}, \vec{v}_{2}, \vec{q}\right)\right\} \epsilon_{i j} \\
& \times \epsilon^{\alpha \beta 3} \psi_{+,-\vec{v}_{i} ; i \alpha}(x) C \psi_{+,-\vec{v}_{j} ; j \beta}(x)-(L \rightarrow R)+\text { h.c. }
\end{aligned}
$$

where

$$
\vec{\alpha}\left(\vec{v}_{1}, \vec{v}_{2}, \vec{q}\right)=2 \vec{q}-\mu_{1} \vec{v}_{1}-\mu_{2} \vec{v}_{2} .
$$

We choose the $z$-axis along $\vec{q}$ and the vectors $\vec{v}_{1}, \vec{v}_{2}$ in the $x-z$ plane; if $\alpha_{1}, \alpha_{2}$ are the angles formed by the vectors $\vec{v}_{1}, \vec{v}_{2}$ with the $z$-axis we have

$$
\begin{aligned}
& \alpha_{x}=-\mu_{1} \sin \alpha_{1} \cos \phi_{1}-\mu_{2} \sin \alpha_{2} \cos \phi_{2}, \\
& \alpha_{y}=-\mu_{1} \sin \alpha_{1} \sin \phi_{1}-\mu_{2} \sin \alpha_{2} \sin \phi_{2}, \\
& \alpha_{z}=2 q-\mu_{1} \cos \alpha_{1}-\mu_{2} \cos \alpha_{2} .
\end{aligned}
$$

In the $\mu_{1}, \mu_{2} \rightarrow \infty$ limit the only non vanishing terms in the sum correspond to the condition

$$
\alpha_{x}=\alpha_{y}=0
$$

Let us introduce

$$
\begin{aligned}
\mu & =\frac{\mu_{1}+\mu_{2}}{2} \\
\delta \mu & =-\frac{\mu_{1}-\mu_{2}}{2} .
\end{aligned}
$$

Eq. 4.19) implies

$$
\alpha_{1}=\alpha_{2}+\pi+\mathcal{O}\left(\frac{1}{\mu}\right)
$$

i.e. the two velocities are opposite in this limit.

The limit $\mu \rightarrow \infty$ does not imply $\alpha_{z}=0$; however if we might take the limit $q \approx \delta \mu \rightarrow \infty$ as well, we would also have $\alpha_{z} \approx 0$; we will make this approximation [34], which is justified by the results of [35]: $q \simeq 1.2 \delta \mu$ and $\delta \mu \sim 0.7 \Delta_{B C S} \gg \Delta^{(s, v)}$. This approximation makes the loop calculations much simpler than the analogous in [35] and therefore it may be particularly useful in complex calculations. In any case corrections to this approximation can be implemented. We have up to terms $\mathcal{O}\left(\frac{1}{\mu^{2}}\right)$ :

$$
\begin{aligned}
& \cos \alpha_{1}=-\frac{\delta \mu}{q}+\frac{q^{2}-\delta \mu^{2}}{q \mu} \\
& \cos \alpha_{2}=+\frac{\delta \mu}{q}+\frac{q^{2}-\delta \mu^{2}}{q \mu},
\end{aligned}
$$

which, together with (4.19), has the solution

$$
\begin{aligned}
\alpha_{2} & \equiv \theta_{q}=\arccos \frac{\delta \mu}{q}-\frac{\epsilon}{2}, \\
\alpha_{1} & =\alpha_{2}+\pi-\epsilon
\end{aligned}
$$


with

$$
\epsilon=2 \frac{\sqrt{q^{2}-\delta \mu^{2}}}{\mu}
$$

Therefore, as anticipated, $\epsilon=\mathcal{O}\left(\frac{1}{\mu}\right)$ and in the limit $\mu \rightarrow \infty$ the two velocities are almost antiparallel:

$$
\vec{v}_{1} \simeq-\vec{v}_{2}
$$

Putting

$$
\psi_{+, \pm \vec{v}_{i} ; i \alpha}(x) \equiv \psi_{ \pm \vec{v}_{i} ; i \alpha}(x)
$$

eq. (4.16) becomes:

$$
\mathcal{L}_{\Delta}^{(s)}=-\frac{\Delta^{(s)}}{2} \sum_{\vec{v}} \epsilon_{i j} \epsilon^{\alpha \beta 3} \psi_{+\vec{v} ; i \alpha}(x) C \psi_{-\vec{v} ; j \beta}(x)-(L \rightarrow R)+\text { h.c. }
$$

In a similar way the term corresponding to the vector condensate in the lagrangian can be written as follows:

$$
\mathcal{L}_{\Delta}^{(v)}=-\frac{\Delta^{(v)}}{2} \sum_{\vec{v}} \sigma_{i j}^{1} \epsilon^{\alpha \beta 3} \psi_{+\vec{v} ; i \alpha}(x) C(\vec{v} \cdot \vec{n}) \psi_{-\vec{v} ; j \beta}(x)-(L \rightarrow R)+\text { h.c. }
$$

where $\vec{n}=\vec{q} /|\vec{q}|$ is the direction corresponding to the total momentum carried by the Cooper pair and we have used $\psi_{-}^{T} C \vec{\alpha} \cdot \vec{n} \psi_{+}=\vec{v} \cdot \vec{n} \psi_{-}^{T} C \psi_{+}$.

In the usual basis of the HDET the effective lagrangian is

$$
\begin{aligned}
\mathcal{L} & =\mathcal{L}_{0}+\mathcal{L}_{\Delta}^{(s)}+\mathcal{L}_{\Delta}^{(v)}= \\
& =\sum_{\vec{v}} \sum_{A=0}^{5} \chi^{A \dagger}\left(\begin{array}{cc}
i \delta_{A B} V \cdot \partial & \Delta_{A B}^{\dagger} \\
\Delta_{A B} & i \delta_{A B} \tilde{V} \cdot \partial
\end{array}\right) \chi^{B} .
\end{aligned}
$$

Here the matrix $\Delta_{A B}$ is as follows:

$$
\Delta_{A B}=0 \quad(A \text { or } B=4 \text { or } 5)
$$

while for $A, B=0, \ldots, 3$ we have:

$$
\Delta_{A B}=\left(\begin{array}{cccc}
\Delta_{0} & 0 & 0 & -\Delta_{1} \\
0 & -\Delta_{0} & -i \Delta_{1} & 0 \\
0 & +i \Delta_{1} & -\Delta_{0} & 0 \\
\Delta_{1} & 0 & 0 & -\Delta_{0}
\end{array}\right)
$$

with

$$
\Delta_{0}=\Delta^{(s)}, \quad \Delta_{1}=\vec{v} \cdot \vec{n} \Delta^{(v)} .
$$

Let us now discuss the precise meaning of the average over velocities. As discussed above we embodied a factor of $1 / 2$ in the average over velocities; however in the LOFF case the sum over the velocities is no longer symmetric and now reads:

$$
\sum_{\vec{v}} \equiv \frac{k_{R}}{2} \int \frac{d \phi}{2 \pi}
$$


if $k_{r}$ is kinematical factor of the order of 1 induced by the approximation of the Riemann-Lebesgue lemma [34] and

$$
\vec{v}=\left(\sin \theta_{q} \cos \phi, \sin \theta_{q} \sin \phi, \cos \theta_{q}\right),
$$

and $\theta_{q}$ given in (4.23). For future reference we observe that

$$
\sum_{\vec{v}} v_{i} v_{j}=k_{R}\left(\frac{\sin ^{2} \theta_{q}}{4}\left(\delta_{i 1} \delta_{j 1}+\delta_{i 2} \delta_{j 2}\right)+\frac{\cos ^{2} \theta_{q}}{2} \delta_{i 3} \delta_{j 3}\right) .
$$

The effective action for the fermi fields in momentum space reads:

$$
S=\sum_{\vec{v}} \sum_{A, B=0}^{5} \int \frac{d^{4} \ell}{(2 \pi)^{4}} \frac{d^{4} \ell^{\prime}}{(2 \pi)^{4}} \chi^{A \dagger}\left(\ell^{\prime}\right) D_{A B}^{-1}\left(\ell^{\prime}, \ell\right) \chi_{B}(\ell),
$$

where $D_{A B}^{-1}\left(\ell^{\prime}, \ell\right)$ is the inverse propagator, given by:

$$
D_{A B}^{-1}\left(\ell^{\prime}, \ell\right)=\left(\begin{array}{cc}
V \cdot \ell \delta_{A B} & \Delta_{A B}^{\dagger} \\
\Delta_{A B} & \tilde{V} \cdot \ell \delta_{A B}
\end{array}\right) \delta^{4}\left(\ell^{\prime}-\ell\right) .
$$

From these equations one can derive the quark propagator, given by

$$
D_{A B}\left(\ell, \ell^{\prime \prime}\right)=(2 \pi)^{4} \delta^{4}\left(\ell-\ell^{\prime \prime}\right) \times \sum_{C}\left(\begin{array}{cc}
\frac{\tilde{V} \cdot \ell \delta_{A C}}{\tilde{D}_{C B}(\ell)} & -\frac{\Delta_{A C}^{\dagger}}{D_{C B}(\ell)} \\
-\frac{\Delta_{A C}}{\tilde{D}_{C B}(\ell)} & \frac{V \cdot \ell \delta_{A C}}{D_{C B}(\ell)}
\end{array}\right)
$$

where

$$
\begin{aligned}
D_{C B}(\ell) & =\left(V \cdot \ell \tilde{V} \cdot \ell-\Delta \Delta^{\dagger}\right)_{C B} \\
\tilde{D}_{C B}(\ell) & =\left(V \cdot \ell \tilde{V} \cdot \ell-\Delta^{\dagger} \Delta\right)_{C B} .
\end{aligned}
$$

The propagator for the fields $\chi^{4,5}$ does not contain gap mass terms and is given by

$$
D\left(\ell, \ell^{\prime}\right)=(2 \pi)^{4} \delta^{4}\left(\ell-\ell^{\prime}\right)\left(\begin{array}{cc}
(V \cdot \ell)^{-1} & 0 \\
0 & (\tilde{V} \cdot \ell)^{-1}
\end{array}\right) .
$$

For the other fields $\chi^{A}, A=0, \cdots, 3$, it is useful to go to a representation where $\Delta \Delta^{\dagger}$ and $\Delta^{\dagger} \Delta$ are diagonal. It is accomplished by performing a unitary transformation which transforms the basis $\chi^{A}$ into the new basis $\tilde{\chi}^{A}$ defined by

$$
\tilde{\chi}^{A}=R_{A B} \chi^{B}
$$

with

$$
R_{A B}=\frac{1}{\sqrt{2}}\left(\begin{array}{cccc}
1 & 0 & 0 & 1 \\
0 & 1 & -i & 0 \\
0 & +i & -1 & 0 \\
1 & 0 & 0 & -1
\end{array}\right)
$$


In the new basis we have

$$
\begin{aligned}
& \left(\Delta^{\dagger}\right)_{A B}=\lambda_{A} \delta_{A B} \\
& \left(\Delta^{\dagger} \Delta\right)_{A B}=\tilde{\lambda}_{A} \delta_{A B}
\end{aligned}
$$

where $\lambda_{A}, \tilde{\lambda}_{A}$ can be approximated as

$$
\begin{aligned}
& \lambda_{A}=\left(\left(\Delta_{0}+\Delta_{1}\right)^{2},\left(\Delta_{0}-\Delta_{1}\right)^{2},\left(\Delta_{0}+\Delta_{1}\right)^{2},\left(\Delta_{0}-\Delta_{1}\right)^{2}\right), \\
& \tilde{\lambda}_{A}=\left(\left(\Delta_{0}-\Delta_{1}\right)^{2},\left(\Delta_{0}-\Delta_{1}\right)^{2},\left(\Delta_{0}+\Delta_{1}\right)^{2},\left(\Delta_{0}+\Delta_{1}\right)^{2}\right) .
\end{aligned}
$$

For further reference we also define

$$
\mu_{C}=\left(\Delta_{0}+\Delta_{1}, \Delta_{1}-\Delta_{0}, \Delta_{0}+\Delta_{1}, \Delta_{1}-\Delta_{0}\right) .
$$

As shown in 34 the integration measure for the LOFF case is

$$
\int \frac{d^{4} \ell}{(2 \pi)^{4}}=\frac{2 \pi \mu^{2}}{(2 \pi)^{3}} \int_{-\delta}^{+\delta} d \ell_{\|} \int_{-\infty}^{+\infty} \frac{d \ell_{0}}{2 \pi}
$$

Let us conclude this section by deriving the gap equation in the LOFF phase. As in the CFL and 2SC cases one writes a truncated Schwinger-Dyson equation. One obtains two equations:

$$
\begin{aligned}
& \Delta_{0}=i \frac{\mu^{2} k_{R}}{24 \Lambda^{2} \pi^{3}} \sum_{A=0}^{3}\left|\mu_{A}\right| I\left(\mu_{A}\right) \\
& \Delta_{1}=-i \frac{\mu^{2} k_{R}}{24 \Lambda^{2} \pi^{3}}\left(\mu_{0} I\left(\mu_{0}\right)+\mu_{1} I\left(\mu_{1}\right)\right),
\end{aligned}
$$

where

$$
I(\Delta)=\int \frac{d^{2} \ell}{V \cdot \ell \tilde{V} \cdot \ell-\Delta^{2}+i \epsilon}=-i 2 \pi \operatorname{arcsinh}\left(\frac{\delta}{|\Delta|}\right) .
$$

The values of the parameters have been fixed in paragraph 2.6. The coupled equations 2.183) have a non trivial solution; one gets [34]:

$$
\begin{aligned}
& \Delta_{0}=\Delta^{(s)}=\frac{\delta}{\sinh \left(\frac{3 \Lambda^{2} \pi^{2}}{\mu^{2} k_{R}}\right)} \\
& \Delta_{1}=\cos \theta_{q} \Delta^{(v)}=0 .
\end{aligned}
$$

These results are compatible with the findings of [35] where the scalar condensate is in the range of a few $\mathrm{MeV}$ and $\Delta^{(v)}$ is found to be negligible. Since in HDET one has $\Delta_{1}=0$ it can be argued that the result $\Delta_{1} \neq 0$ found in 35] comes from a non leading $\mathcal{O}\left(\frac{1}{\mu}\right)$ contribution. 


\subsection{Phonon-quark coupling}

Using the results of the two previous sections one gets the following 3-point and 4-point phonon-quark-quark couplings:

$$
\begin{aligned}
\mathcal{L}_{\phi \psi \psi}= & -\frac{i \phi}{f} \sum_{\vec{v}}\left[\Delta^{(s)} \epsilon_{i j}+\vec{v} \cdot \vec{n} \Delta^{(v)} \sigma_{i j}^{1}\right] \epsilon^{\alpha \beta 3} \psi_{i, \alpha, \vec{v}} C \psi_{j, \beta,-\vec{v}} \\
& -\frac{1}{2 f q} \sum_{\vec{v}} \vec{v} \cdot[\vec{\nabla} \phi-\vec{n}(\vec{n} \cdot \vec{\nabla} \phi)] \Delta^{(v)} \sigma_{i j}^{1} \epsilon^{\alpha \beta 3} \psi_{i, \alpha, \vec{v}} C \psi_{j, \beta,-\vec{v}} \\
& -(L \rightarrow R)+\text { h.c. }
\end{aligned}
$$

and

$$
\begin{aligned}
\mathcal{L}_{\phi \phi \psi \psi}= & \frac{\phi^{2}}{2 f^{2}} \sum_{\vec{v}}\left[\Delta^{(s)} \epsilon_{i j}+\vec{v} \cdot \vec{n} \Delta^{(v)} \sigma_{i j}^{1}\right] \epsilon^{\alpha \beta 3} \psi_{i, \alpha, \vec{v}} C \psi_{j, \beta,-\vec{v}} \\
& -\frac{i \phi}{f} \sum_{\vec{v}} \vec{v} \cdot[\vec{\nabla} \phi-\vec{n}(\vec{n} \cdot \vec{\nabla} \phi)] \Delta^{(v)} \sigma_{i j}^{1} \epsilon^{\alpha \beta 3} \psi_{i, \alpha, \vec{v}} C \psi_{j, \beta,-\vec{v}} \\
& -\frac{1}{4 f^{2} q^{2}} \sum_{\vec{v}}\left[\frac{\vec{v} \cdot \vec{n}}{2}\left(3(\vec{n} \cdot \vec{\nabla} \phi)^{2}-|\vec{\nabla} \phi|^{2}\right)\right. \\
- & (\vec{v} \cdot \vec{\nabla} \phi)(\vec{n} \cdot \vec{\nabla} \phi)] \times \\
& \times \Delta^{(v)} \sigma_{i j}^{1} \epsilon^{\alpha \beta 3} \psi_{i, \alpha, \vec{v}} C \psi_{j, \beta,-\vec{v}}-(L \rightarrow R)+\text { h.c. }
\end{aligned}
$$

In the basis of the $\chi$ fields one gets

$$
\mathcal{L}_{3}+\mathcal{L}_{4}=\sum_{\vec{v}} \sum_{A=0}^{3} \tilde{\chi}^{A \dagger}\left(\begin{array}{cc}
0 & g_{3}^{\dagger}+g_{4}^{\dagger} \\
g_{3}+g_{4} & 0
\end{array}\right) \tilde{\chi}^{B} .
$$

Here

$$
\begin{aligned}
& g_{3}=\left[\frac{i \phi \Delta_{A B}}{f}+\sigma_{A B} \hat{O}[\phi]\right], \\
& g_{4}=\left[-\frac{\phi^{2} \Delta_{A B}}{2 f^{2}}+\sigma_{A B}\left(\frac{i \phi}{f} \hat{O}[\phi]+\hat{Q}[\phi]\right)\right],
\end{aligned}
$$

with

$$
\begin{gathered}
\hat{O}[\phi]=\frac{1}{2 f q} \vec{v} \cdot[\vec{\nabla} \phi-\vec{n}(\vec{n} \cdot \vec{\nabla} \phi)] \Delta^{(v)} \\
\hat{Q}[\phi]=\frac{\Delta^{(v)}}{4 f^{2} q^{2}}\left[\frac{\vec{v} \cdot \vec{n}}{2}\left(3(\vec{n} \cdot \vec{\nabla} \phi)^{2}-|\vec{\nabla} \phi|^{2}\right)-(\vec{v} \cdot \vec{\nabla} \phi)(\vec{n} \cdot \vec{\nabla} \phi)\right], \\
\sigma_{A B}=\left(\begin{array}{cccc}
0 & 0 & 0 & -1 \\
0 & 0 & -i & 0 \\
0 & +i & 0 & 0 \\
+1 & 0 & 0 & 0
\end{array}\right) .
\end{gathered}
$$

Finally the effective action for the field $\phi, S[\phi]$, is obtained by the lagrangian as follows

$$
S=\int d t d x d y \frac{\pi}{q} \sum_{k=-\infty}^{+\infty} \mathcal{L}(\phi(t, x, y, k \pi / q) .
$$




\subsection{Dispersion law for the phonon field}

To introduce formally the NGB in the theory one uses the same gradient expansion discussed in the paragraph 2.3.1. At the lowest order one has to consider the diagrams in Fig. 2.1, i.e. the self-energy and the tadpole diagrams. At the second order in the momentum expansion one gets, after averaging over the Fermi velocities

$$
\Pi(p)=-\frac{\mu^{2} k_{R}}{4 \pi^{2} f^{2}}\left[p_{0}^{2}-v_{\perp}^{2}\left(p_{x}^{2}+p_{y}^{2}\right)-v_{\|}^{2} p_{z}^{2}\right] .
$$

One obtains canonical normalization for the kinetic term provided

$$
f^{2}=\frac{\mu^{2} k_{R}}{2 \pi^{2}}
$$

On the other hand, at the lowest order in $\Delta^{(v)}$ :

$$
\begin{aligned}
v_{\perp}^{2} & =\frac{1}{2} \sin ^{2} \theta_{q}+\left(1-3 \cos ^{2} \theta_{q}\right)\left(1-\log \frac{2 \delta}{\Delta_{0}}\right)\left(\frac{\Delta^{(v)}}{q}\right)^{2} \\
v_{\|}^{2} & =\cos ^{2} \theta_{q} .
\end{aligned}
$$

In conclusion, the dispersion law for the phonon is

$$
E(\vec{p})=\sqrt{v_{\perp}^{2}\left(p_{x}^{2}+p_{y}^{2}\right)+v_{\|}^{2} p_{z}^{2}}
$$

which is anisotropic. Besides the anisotropy related to $v_{\perp} \neq v_{\|}$, there is another source of anisotropy, due to the fact that $p_{z}$, the component of the momentum perpendicular to the planes (4.5), differently from $p_{x}$ and $p_{y}$ is a quasi momentum and not a real momentum. 


\section{Chapter 5}

\section{Astrophysical implications}

\subsection{Overview}

Should we look for a laboratory to test color superconductivity, we would face the problem that in the high energy physics programmes aiming at new states of matter, such as the Quark Gluon Plasma, the region of the $T-\mu$ plane under investigation is that of low density and high temperature. On the contrary we need physical situations characterized by low temperature and high densities. These conditions are supposed to occur in the inner core of neutron stars, under the hypothesis that, at the center of these compact stars, nuclear matter has become so dense as to allow the transition to quark matter. A schematic view of a neutron star is in Fig. 5.1. In the subsequent sections we shall give a

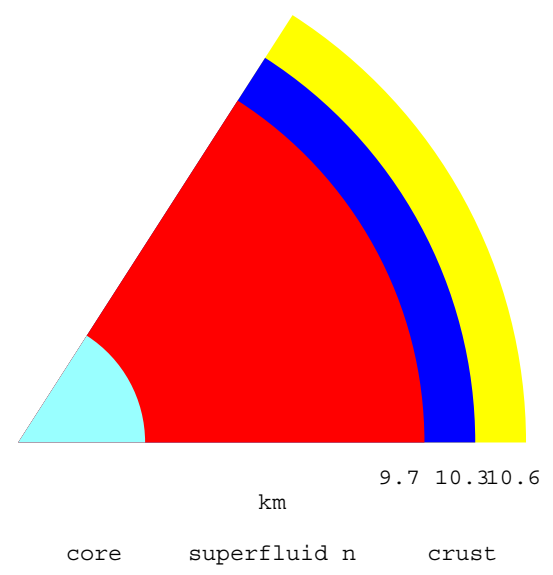

Fig. 5.1 Schematic view of a neutron star as computed by an equation of state with three nucleon interaction 75 .

pedagogical introduction to the physics of compact stars and we shall review some possible astrophysical implications of the color superconductivity. 


\subsection{A brief introduction to compact stars}

To begin with, let us show that for a fermion gas high chemical potential means high density. To simplify the argument we assume that the fermions are massless and not interacting, so that $\mu=\epsilon_{F}=p_{F}$. It follows from eq. (1.8) that

$$
\rho \propto \mu^{3}
$$

which means that the chemical potential increases as $\rho^{1 / 3}$ : This is the reason why we should search color superconductivity in media with very high baryonic density.

The equations (1.8)-(1.10) allow to determine the equation of state, which is obtained by solving the equations

$$
P=\Phi\left(x_{F}\right), \quad \rho \propto x_{F}^{3},
$$

where $x_{F}=p_{F} / m c$. For the simplest case (free massless fermions) we obtain

$$
P \propto \mu^{4}, \quad \rho \propto \mu^{3},
$$

and therefore the equation of state has the form

$$
P=K \rho^{\frac{4}{3}}
$$

Clearly this result also holds for massive fermions in the Ultra Relativistic (UR) case where the mass is negligible. One can prove that in the Non Relativistic (NR) case one has $P=K \rho^{\frac{5}{3}}$. More generally the equation of state can be approximated by the expression

$$
P=K \rho^{\gamma}
$$

and the two cases discussed above are characterized as follows:

$$
\begin{array}{cl}
N R\left(\gamma=\frac{5}{3}\right) & \left.\rho \ll 10^{6} \mathrm{~g} / \mathrm{cm}^{3} \text { \{electrons }\right\} \\
& \left.\rho \ll 10^{15} \mathrm{~g} / \mathrm{cm}^{3} \text { \{neutrons }\right\} \\
U R\left(\gamma=\frac{4}{3}\right) & \rho \gg 10^{6} \mathrm{~g} / \mathrm{cm}^{3},\{\text { electrons }\} \\
& \left.\rho \gg 10^{15} \mathrm{~g} / \mathrm{cm}^{3} \text { \{neutrons }\right\} .
\end{array}
$$

Let us note explicitly that, at $T=0, P \neq 0$. This is a quantum-mechanical effect due to the Pauli principle and the Fermi Dirac statistics (for comparison, for a classical Maxwell Boltzmann gas $P \rightarrow 0$ when $T \rightarrow 0$ ). In absence of other sources of outward pressure it is the pressure of the degenerate fermion gas that balances the gravity and avoids the stellar collapse. 
One can see that the densities that can be reached in the compact stars are very different depending on the nature of the fermions. The two cases correspond to two classes of compact stars, the white dwarfs and the neutron stars. White dwarfs (w.d.s) are stars that have exhausted nuclear fuel; well known examples are Sirius B, or 40 Eri B. In the Hertzsprung-Russel diagram w.d.s fill in a narrow corner below the main sequence. In a w.d. stellar equilibrium is reached through a compensation between the inward pressure generated by gravity and the outward the pressure of degenerate electrons. Typical values of the central density, mass and radius for a w.d. are $\rho=10^{6} \mathrm{~g} / \mathrm{cm}^{3}, M \sim M_{\odot}$, $R \sim 5,000 \mathrm{~km}$.

Suppose now that in the star higher values of $\rho$ are reached. If $\rho$ increases, inverse beta decay becomes important:

$$
e^{-} p \rightarrow n \nu .
$$

This process fixes the chemical composition at equilibrium

$$
\mu_{e}+\mu_{p}=\mu_{n}
$$

On the other hand one has to enforce neutrality:

$$
n_{e}=n_{p} .
$$

One proves that these two conditions fix the ratio of the number of proton $n_{p}$ to the number of neutrons $n_{n}$ for ultrarelativistic particles:

$$
\frac{n_{p}}{n_{n}}=\frac{1}{8}
$$

This number should be seen as a benchmark value, as it is derived under simplifying hypotheses, most notably the absence of interactions and the neglect of masses. In any event it suggests that, for higher densities, the star tends to have a relatively larger fraction of neutrons and therefore it is named a neutron star. It must be stressed that one of the relevant facts about neutron stars is that the general relativity effects cannot be ignored and the relevant equilibrium equations to be used are the Oppenheimer-Volkov equations of hydrostatic equilibrium.

The following simple argument, due to Landau (1932) can be used to evaluate the relevant parameters of white dwarfs and neutron stars (see the textbook [75]; more recent reviews of compact stars are in [76]). Let us consider $N$ fermions in a sphere of radius $R$ at $T=0$; the number of fermion per volume unit scales as $n \sim N / R^{3}$; the volume per fermion is therefore $\sim \frac{1}{n}$ and the uncertainty on the position is of the order of $n^{-1 / 3}$; the Fermi momentum is of the order of the uncertainty on the fermion momentum and therefore

$$
p_{F} \sim n^{1 / 3} \hbar,
$$

a result we obtained already under more stringent hypotheses (Fermi-Dirac distribution) and derived again here using only the uncertainty relations. The Fermi energy of the baryons is therefore

$$
\epsilon_{F} \sim \frac{\hbar c N^{1 / 3}}{R}
$$


if $N$ is the total number of baryons. Note that this applies both to neutron stars and to electron stars, because also in stars where the pressure mainly come from electrons there will be a considerable amount of protons and neutrons and the largest part of the energy comes from the baryons, not from the electrons. On the other hand the gravitational energy per baryon is

$$
E_{G} \sim-\frac{G N m_{B}^{2}}{R}
$$

and the total energy can be estimated as

$$
E=E_{G}+E_{F} \sim \frac{\hbar c N^{1 / 3}}{R}-\frac{G N m_{B}^{2}}{R} .
$$

Now equilibrium can exist only if $E \geq 0$. As a matter of fact if $E<0$ ( $N$ large) then $\lim _{R \rightarrow 0} E=-\infty$, which means that the energy is unbounded from below and the system is unstable. Therefore, $E \geq 0$ gives the maximum number of baryons as follows:

$$
N \leq N_{\max }=\left(\frac{\hbar c}{G m_{B}^{2}}\right)^{3 / 2} \sim 2 \times 10^{57} .
$$

As a consequence the maximum mass is

$$
M_{\text {max }}=N_{\max } m_{B}=1.5 M_{\odot} .
$$

This mass can be estimated better and its better determination $\left(\sim 1.4 M_{\odot}\right)$ is known as the Chandrasekhar limit; for our purposes the estimate (5.12) is however sufficient. Notice that the Chandrasekhar limit is similar for compact stars where the degeneracy pressure is mainly supplied by electrons and those where it is supplied by baryons.

One can also estimate the radius of a star whose mass is given by (5.12). One has

$$
\epsilon_{F} \geq m c^{2} \sim \frac{\hbar c}{R} N_{\text {max }}^{1 / 3} \sim \frac{\hbar c}{R}\left(\frac{\hbar c}{G m_{B}^{2}}\right)^{1 / 2}
$$

and, therefore,

$$
R \sim \frac{\hbar}{m c}\left(\frac{\hbar c}{G m_{B}^{2}}\right)^{1 / 2}=\left\{\begin{array}{l}
5 \times 10^{8} \mathrm{~cm}\left\{m=m_{e}\right\} \\
3 \times 10^{5} \mathrm{~cm}\left\{m=m_{n}\right\}
\end{array}\right.
$$

If a neutron star accretes its mass beyond the Chandrasekhar limit nothing can prevent the collapse and it becomes a black holef. In the following table we summarize our discussion; notice that we report for the various stars also the value of the parameter $G M / R c^{2}$ i.e. the ratio of the Schwarzschild radius to the star's radius. Its smallness measures the validity of the approximation of neglecting the general relativity effects; one can see that for the sun and the white dwarfs the newtonian treatment of gravity represents a fairly good approximation.

\footnotetext{
${ }^{1}$ The exact determination of the mass limit depends on the model for nuclear forces; for example in 77 the neutron star mass limit is increased beyond $1.4 M_{\odot}$.
} 


\begin{tabular}{|c|c|c|c|c|}
\hline & $M$ & $R$ & $\rho\left(\frac{g}{c^{3}}\right)$ & $\frac{G M}{R c^{2}}$ \\
\hline Sun & $M_{\odot}$ & $R_{\odot}$ & 1 & $10^{-6}$ \\
White Dwarf & $\leq M_{\odot}$ & $10^{-2} R_{\odot}$ & $\leq 10^{7}$ & $10^{-4}$ \\
Neutron Star & $1-3 M_{\odot}$ & $10^{-5} R_{\odot}$ & $\leq 10^{15}$ & $10^{-1}$ \\
Black Hole & arbitrary & $\frac{2 G M}{c^{2}}$ & $\sim \frac{M}{R^{3}}$ & 1 \\
\hline
\end{tabular}

Table 5.1 Paramers of different stellar objects.

Neutron stars are the most likely candidate for the theoretical description of pulsars. Pulsars are rapidly rotating stellar objects, discovered in 1967 by Hewish and collaborators and identified as rotating neutron stars by Gold [78]; so far about 1200 pulsars have been identified.

Pulsars are characterized by the presence of strong magnetic fields with the magnetic and rotational axis misaligned; therefore they continuously emit electromagnetic energy (in the form of radio waves) and constitute indeed a very efficient mean to convert rotational energy into electromagnetic radiation. The rotational energy loss is due to dipole radiation and is therefore given by

$$
\frac{d E}{d t}=I \omega \frac{d \omega}{d t}=-\frac{B^{2} R^{6} \omega^{4} \sin ^{2} \theta}{6 c^{3}} .
$$

Typical values in this formula are, for the moment of inertia $I \sim R^{5} \rho \sim$ $10^{45} \mathrm{~g} / \mathrm{cm}^{3}$, magnetic fields $B \sim 10^{12} \mathrm{G}$, periods $T=2 \pi / \omega$ in the range 1.5 msec-8.5 sec; these periods increase slowly 9 , with derivatives $\frac{d T}{d t} \sim 10^{-12}$ $10^{-21}$, and never decrease except for occasional jumps (called glitches).

Glitches were first observed in the Crab and Vela pulsars in 1969; the variations in the rotational frequency are of the order $10^{-8}-10^{-6}$.

This last feature is the most significant phenomenon pointing to neutron stars as a model of pulsars in comparison to other form of hadronic matter, such as strange quarks. It will be discussed in more detail in par. 5.6, where we will examine the possible role played by the crystalline superconducting phase. In the subsequent three paragraphs we will instead deal with other possible astrophysical implications of color superconductivity.

\footnotetext{
${ }^{2}$ Rotational period and its derivative can be used to estimate the pulsar's age by the approximate formula $\frac{T}{2 \frac{d T}{d t}}$, see e.g. 79.
} 


\subsection{Supernovae neutrinos and cooling of neutron stars}

Neutrino diffusion is the single most important mechanism in the cooling of young neutron stars, i.e. with an age $<10^{5}$ years; it affects both the early stage and the late time evolution of these compact stars. To begin with let us consider the early evolution of a Type II Supernova.

Type II supernovae are supposed to be born by collapse of massive $(M \sim$ $\left.8-20 M_{\odot}\right)$ stars $^{3}$. These massive stars have unstable iron cores ${ }^{\text {t }}$ with masses of the order of the Chandrasekhar mass. The explosion producing the supernova originates within the core, while the external mantle of the red giant star produces remnants that can be analyzed by different means, optical, radio and $\mathrm{X}$ rays. These studies agree with the hypothesis of a core explosion. The emitted energy $\left(\sim 10^{51} \mathrm{erg}\right)$ is much less than the total gravitational energy of the star, which confirms that the remnants are produced by the outer envelope of the massive star; the bulk of the gravitational energy, of the order of $10^{53} \mathrm{erg}$, becomes internal energy of the proto neutron star (PNS). The suggestion that neutron stars may be formed in supernovae explosions was advanced in 1934 by Baade and Zwicky [80] and it has been subsequently confirmed by the observation of the Crab pulsar in the remnant of the Crab supernova observed in China in 1054 A.D.

We do not proceed in this description as it is beyond the scope of this review and we concentrate our attention on the cooling of the PNS 9, which is mostly realized through neutrino diffusion. By this mechanism one passes from the initial temperature $T \sim 20-30 \mathrm{MeV}$ to the cooler temperatures of the neutron star at subsequent stages. This phase of fast cooling lasts 10-20 secs and the neutrinos emitted during it have mean energy $\sim 20 \mathrm{MeV}$. These properties, that can be predicted theoretically, are also confirmed by data from SN 1987A.

The role of quark color superconductivity at this stage of the evolution of the neutron stars has been discussed in [82]. In this paper the neutrino mean free path is computed in a color super-conducting medium made up by quarks in two flavor (2SC model). The results obtained indicate that the cooling process by neutrino emission slows down when the quark matter undergoes the phase transition to the superconducting phase at the critical temperature $T_{c}$, but then accelerates when $T$ decreases below $T_{c}$. There should be therefore changes in the neutrino emission by the PNS and they might be observed in some future supernova event; this would produce an interesting test for the existence of a color superconducting phase in compact stars.

Let us now consider the subsequent evolution of the neutron star, which also depends on neutrino diffusion. The simplest processes of neutrino production are the so called direct Urca processes

$$
f_{1}+\ell \rightarrow f_{2}+\nu_{\ell}
$$

\footnotetext{
${ }^{3}$ The other supernovae, i.e. type I supernovae, result from the complete explosion of a star with $4 M_{\odot} \leq M \leq 8 M_{\odot}$ with no remnants.

${ }^{4}$ Fusion processes favor the formation of iron, as the binding energy per nucleon in nuclei has a maximum for $A \sim 60$.

${ }^{5}$ See 81] for further discussions.
} 


$$
f_{2} \rightarrow f_{1}+\ell \rightarrow f_{2}+\bar{\nu}_{\ell}
$$

by these reactions, in absence of quark superconductivity, the interior temperature $T$ of the star drops below $10^{9} \mathrm{~K}(\sim 100 \mathrm{KeV})$ in a few minutes and in $10^{2}$ years to temperatures $\sim 10^{7} \mathrm{~K}$. Generally speaking the effect of the formation of gaps is to slow down the cooling, as it reduces both the emissivity and the specific heat. However not only quarks, if present in the neutron star, but also other fermions, such as neutrons, protons or hyperons have gaps, as the formation of fermion pairs is unavoidable if there is an attractive attraction in any channel (see the discussion in par. 1.3). Therefore, besides quark color superconductivity, one has also the phenomenon of baryon superconductivity and neutron superfluidity, which is the form assumed by this phenomenon for neutral particles. The analysis is therefore rather complicated; the thermal evolution of a late time neutron star has been discussed in [83], but no clear signature for the presence of color superconductivity seems to emerge from the theoretical simulations and, therefore, one may tentatively conclude that the late time evolution of the neutron stars does not offer a good laboratory to test the existence of color superconductivity in compact stars.

\section{$5.4 R$-mode instabilities in neutron stars and strange stars}

Rotating relativistic stars are in general unstable against the rotational mode ( $r$-mode instability) 84]. The instability is due to the emission of angular momentum by gravitational waves from the mode. Unless it is damped by viscosity effects, this instability would spin down the star in relatively short times. More recently it has been realized [85] that in neutron stars there is an important viscous interaction damping the $r$-mode i.e. that between the external metallic crust and the neutron superfluid. The consequence of this damping is that $r$-modes are significant only for young neutron stars, with periods $T<2 \mathrm{msec}$. For larger rotating periods the damping of the $r$-mode implies that the stars slow down only due to magnetic dipole braking.

All this discussion is relevant for the nature of pulsars: Are they neutron stars or strange stars?

The existence of strange stars, i.e. compact stars made of quarks $u, d, s$ in equal ratios would be a consequence of the existence of stable strangelets. This

hypothetical form of nuclear matter, made of a large number of $u, d, s$ quarks has been suggested by Bodmer [86] and Witten [87] as energetically favored in comparison to other hadronic phases when a large baryonic number is involved. The reason is that in this way the fermions, being of different flavors, could circumvent the Pauli principle and have a lower energy, in spite of the larger strange quark mass. If strangelets do exist, basically all the pulsars should be strange stars because the annihilation of strange stars, for example from a binary system, would fill the space around with strangelets that, in turn, would convert ordinary nuclear stars into strange stars. 
An argument in favor of the identification of pulsars with strange stars is the scarcity of pulsars with very high frequency $(T<2.5 \mathrm{msec})$.

This seems to indicate that indeed the $r$-mode instability is effective in slowing down the compact stars and favors strange stars, where, differently from neutron stars, the crust can be absent. Even in the presence of the external crust, that in a quark star can be formed by the gas after the supernova explosion or subsequent accretion, the dampening of the $r$-mode is less efficient. As a matter of fact, since electrons are only slightly bounded, in comparison with quarks that are confined, they tend to form an atmosphere having a thickness of a few hundred Fermi; this atmosphere produces a separation between the nuclear crust and the inner quark matter and therefore the viscosity is much smaller.

In quark matter with color superconductivity the presence of gaps $\Delta \gg T$ exponentially reduces the bulk and shear viscosity, which renders the $r$-mode unstable. According to Madsen 88, 89] this would rule out compact stars entirely made of quarks in the CFL case (the 2SC model would be marginally compatible, as there are ungapped quarks in this case). For example for $\Delta>1$ $\mathrm{MeV}$ any star having $T<10$ msec would be unstable, which would contradict the observed existence of pulsars with time period less than $10 \mathrm{msec}$.

However this conclusion does not rule out the possibility of neutron stars with a quark core in the color superconducting state, because as we have stressed already, for them the dampening of the $r$-mode instability would be provided by the viscous interaction between the nuclear crust and the neutron superfluid.

\subsection{Miscellaneous results}

Color superconductivity is a Fermi surface phenomenon and as such, it does not affect significantly the equation of state of the compact star. Effects of this phase could be seen in other astrophysical contexts, such as those considered in the two previous paragraphs or in relation to the pulsar glitches, which will be examined in the next paragraph. A few other investigations have been performed in the quest of possible astrophysical signatures of color superconductivity; for instance in [56] it has been suggested that the existence of a 2SC phase might be partly responsible of the gamma ray bursts, due to the presence in the two-flavor superconducting phase of a light glueball that can decay into two photons. Another interesting possibility is related to the stability of strangelets, because, as observed in [90], CFL strangelets, i.e. lumps of strange quark matter in the CFL phase may be significantly more stable than strangelets without color superconductivity.

Finally I wish to mention the observation of 11] concerning the evolution of the magnetic field in the interior of neutron stars. Inside an ordinary neutron

star, neutron pairs are responsible for superfluidity, while proton pairs produce BCS superconductivity. In this condition magnetic fields experience the ordinary Meissner effect and are either expelled or restricted to flux tubes where there is no pairing. In the CFL (and also 2SC) case, as we know from paragraph 
3.1.2, a particular $U(1)$ group generated by

$$
\tilde{Q}=\mathbf{1} \otimes Q+T \otimes \mathbf{1}
$$

remains unbroken and plays the role of electromagnetism. Instead of being totally dragged out or confined in flux tubes, the magnetic field will partly experience Meissner effect (the component $\tilde{A}_{\mu}$ ), while the remaining part will remain free in the star. During the slowing down this component of the magnetic field should not decay because, even though the color superconductor is not a BCS conductor for the group generated by (5.16), it may be a good conductor due to the presence of the electrons in the compact star. Therefore it has been suggested [11] that a quark matter core inside a neutron star may serve as an "anchor" for the magnetic field.

\subsection{Glitches in neutron stars}

Glitches are a typical phenomenon of of the pulsars, in the sense that probably all the pulsar have glitches (for a recent review see 91]). Several models have been proposed to explain the glitches. Their most popular explanation is based on the idea that these sudden jumps of the rotational frequency are due to the the angular momentum stored in the superfluid neutrons in the inner crust (see Fig. 5.1), more precisely in vortices pinned to nuclei. When the star slows down, the superfluid neutrons do not participate in the movement, until the state becomes unstable and there is a release of angular momentum to the crust, which is seen as a jump in the rotational frequency.

The presence of glitches is one of the main reasons for the identification of pulsars with neutron stars; as a matter of fact neutron stars are supposed to have a dense metallic crust, differently from quark stars where the crust is absent or, if present, is much less dense $\left(\approx 10^{11} \mathrm{~g} \mathrm{~cm}^{-3}\right)$.

A schematic and a somehow exaggerated diagram of glitches is shown in fig. 5.2. The interesting aspect of the LOFF phase discussed in chapter 1 is that even in quark stars, provided we are in a color superconductivity phase, one can have a crystal structure able to produce glitches; it would be given by a lattice characterized by a geometric array where the gap parameter varies periodically. This would avoid the objection raised in [92] that excludes the existence of strange stars because, if the strange matter exists, strange stars should be rather common, as we discussed in par. 5.4, in contrast with the widespread appearance of glitches in pulsars. Therefore, if the color crystalline structure is able to produce glitches, the argument in favor of the existence of strange stars would be reinforced.

In a more conservative vein one can also imagine that the LOFF phase be realized in the inner core of neutron star; in this case the crystalline color superconductivity could be partly responsible for the glitches of the pulsar. Some estimates of the effect are discussed in [35]; a detailed analysis of this scenario is however premature as one should first complete the study of the LOFF phase in two directions, first by including the third quark (all the study in section 6 was relative to the two flavor case) and, second, by sorting out the 


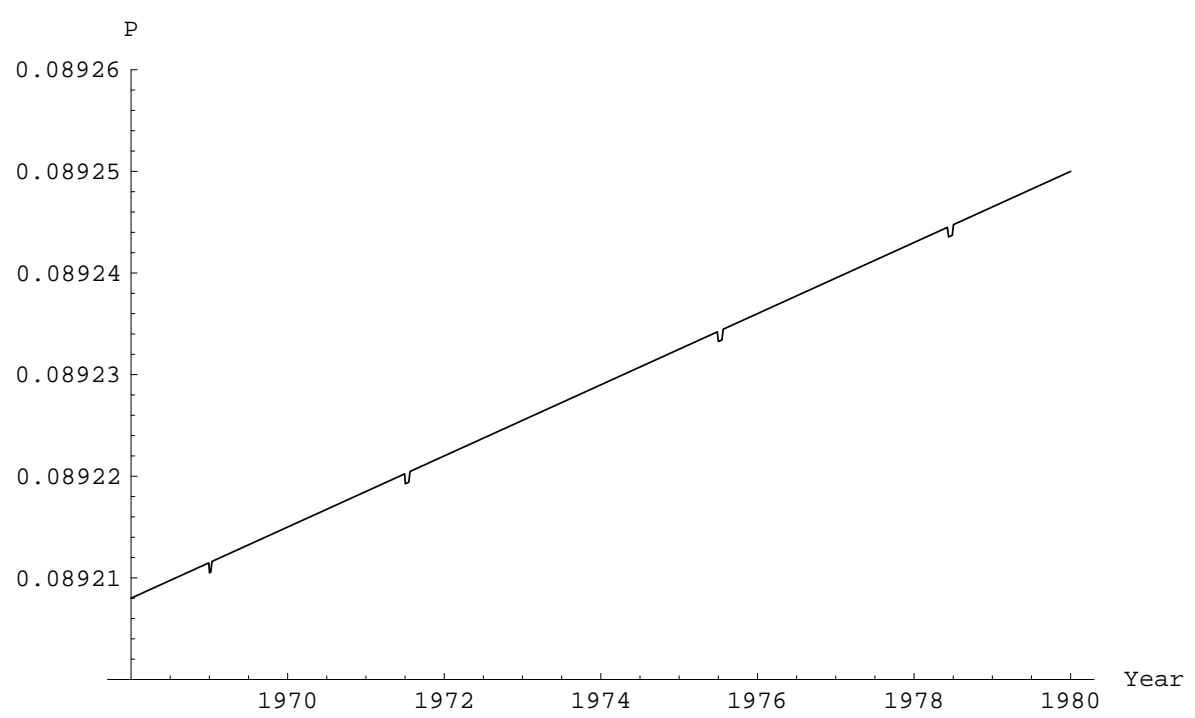

Fig. 5.2 Schematic view of the period $P$ of the PSR 0833-45 (Vela) showing four glitches in the years 1969-1980. Other glitches have been observed in the subsequent years.

exact form of the color lattice, as the plane wave ansatz discussed in section 4 might not correspond to the true vacuum of the theory.

\section{Acknowledgements}

I would like to thank R. Casalbuoni for reading the manuscript and for several long-distance, long-lasting calls that helped me in understanding the subject of this review. I am grateful to R. Gatto and M. Mannarelli for a very pleasant scientific collaboration on the themes of this review. It is also a pleasure to thank K. Rajagopal for many enlightening discussions on color superconductivity, I. Bombaci for a useful correspondence on the astrophysical consequences of the LOFF phase and J. R. Peláez for useful discussions. 


\section{Bibliography}

[1] BARROIS B.: Nucl. Phys. B, 129, (1977) 390; S. FRAUTSCHI, in Proceedings of the workshop on hadronic matter at extreme density, Erice 1978. See also: BAILIN D. AND LOVE A.: Phys. Rept., 107, (1984) 325, and references therein.

[2] Alford M., RAJAgOPAL K. AND WILCZEK F.: Phys. Lett. B, 422, (1998) 247 arXiv:hep-ph/9711395.

[3] Alford M., RAJAGOPAL K. AND WILCZEK F: Nucl. Phys. B, 537, (1999) 443 arXiv:hep-ph/9804403.

[4] AlFord M., BERGES J. AND RAJAGOPAL K.: Nucl. Phys. B, 558, (1999) 219 arXiv:hep-ph/9903502.

[5] SCHÄFER T. AND WILCZEK F.: Phys. Rev. Lett., 82, (1999) 3956 arXiv:hep-ph/9811473.

[6] SCHÄFER T. AND WILCZEK F.: Phys. Rev. D, 60, (1999) 114033 arXiv:hep-ph/9906512.

[7] SCHÄFER T. AND WILCZEK F.: Phys. Rev. D, 60, (1999) 074014 arXiv:hep-ph/9903503.

[8] SCHAFER T. AND WILCZEK F.: Phys. Lett. B, 450, (1999) 325 arXiv:hep-ph/9810509.

[9] CARTER G. W. AND DIAKONOV D: Phys. Rev. D, 60, (1999) 16004 arXiv:hep-ph/9812445.

[10] RAPP R., SCHÄFER T., SHURYAK E. V. AND M. VELKOVSKY M.: Phys. Rev. Lett., 81, (1998) 53 arXiv:nucl-th/9711396.

[11] Alford M., Berges J. AND RAJAGOPAL K.: Nucl. Phys. B, 571, (2000) 269 arXiv:hep-ph/9910254.

[12] PISARSKI R. D. AND RISCHKE D.H.: Phys. Rev. Lett., 83, (1999) 37 arXiv:nucl-th/9811104; Phys. Rev. D, 61, (2000) 051501 arXiv:nuclth/9907041].

[13] AGASIAN N. O., KERBIKOV B.O. AND SCHEVCHENKO V. I.: Phys. Rep., 320, (1999) 131 arXiv:hep-ph/9902335; SHUSTER E. AND SON 
D. T.: Nucl. Phys., 573, (2000) 434 arXiv:hep-ph/9905448; HONG D. K., MIRANSKY V. A., SHOVKOVY I. A. AND WIJEWARDHANA L. C. R.: Phys. Rev. D, 61, (2000) 056001, erratum, ibidem, 62, (2000) 59903 arXiv:hep-ph/9906478.

[14] RAJAGOPAL K. AND F. WILCZEK F.: in Handbook of $Q C D$, M.Shifman, ed. (World Scientific, 2001) arXiv:hep-ph/0011333.

[15] HONG D. K.: arXiv:hep-ph/0101025; ALFORD M.: arXiv:hep$\mathrm{ph} / 0102047$.

[16] HSU S. D. H.: arXiv:hep-ph/0003140.

[17] BARDEEN J., COOPER L.N. AND SCHRIEFFER J. R.: Phys. Rev., 106, (1957) 162; 108, (1957) 1175; see also SCHRIEFFER J. R.: Theory of Superconductivity (Benjamin/Cummings, Menlo Park) 1964.

[18] EBERT D., KHUDYAKOV V. V., ZHUKOVSKY V. C. AND KLIMENKO K. G.: Phys. Rev. D 65, 054024 (2002) arXiv:hep-ph/0106110; EBERT D., KHUDYAKOV V. V. , KLIMENKO K. G., TOKI H. AND ZHUKOVSKY V. C.: arXiv:hep-ph/0108185.

[19] CASAlbuOni R., DUAn Z. And SAnNino F., Phys. Rev. D, 62, (2000) 094004 arXiv:hep-ph/0004207.

[20] CASAlBuOni R. AND GATTO R.: Phys. Lett. B, 464, (1999) 111 arXiv:hep-ph/9908227.

[21] POLCHINSKI J.: in Proc. of the 1992 TASI, eds. J. Harvey and J. Polchinski (Singapore, 1993) arXiv:hep-th/9210046.

[22] SHANKAR R.: Rev. Mod. Phys. , 66, (1994) 129.

[23] Benfatto G. AND Gallavotti G.: J. Stat. Phys., 59, (1990) 541; Phys. Rev. C 42, (1990) 9967.

[24] WILSON K. G.: Phys. Rev. B, 4, (1981) 3174; 4, (1981) 3184.

[25] See e.g. ABRIKOSOV A. A., GORKOV L. P. AND DZYALOSHINSKI I. E.: Methods of Quantum Field Theory in Statistical Mechanics (Dover, New York) 1963.

[26] EVAnS N., HSU S. D. AND SCHWETZ M.: Nucl. Phys. B, 551, (1999) 275 arXiv:hep-ph/9808444.

[27] HONG D. K.: Phys. Lett. B, 473, (2000) 118 [arXiv: hep-ph/9812510]; HONG D. K.: Nucl. Phys. B, 582, (2000) 451 arXiv:hep-ph/9905523.

[28] BeAne S. R., BedAque P. F. And SAVAGE M. J.: Phys. Lett. B, 483, (2000) 131 arXiv:hep-ph/0002209

[29] CASAlBuOni R., GATtO R. AND NARDulli G.: Phys. Lett. B, 498, (2001) 179 arXiv:hep-ph/0010321]. 
[30] For reviews of the heavy quark effective theory see for example the textbook: MANOHAR A. V. AND WISE M. B.: Heavy Quark Physics (Cambridge Univ. Press, 2000) and CASALBUONI R. ET AL.: Phys. Rept., 281, (1997) 145 arXiv:hep-ph/9605342.

[31] CASALBUONI R., GATTO R., MANNARELLI M. AND NARDULli G.: Phys. Lett. B, 524, (2002) 144 arXiv:hep-ph/0107024].

[32] CASAlBUONI R., GATTO R., MANNARELLI M. AND NARDULLI G.: Phys. Lett. B 511, (2001) 218 arXiv:hep-ph/0101326.

[33] NARDULLI G.: arXiv:hep-ph/0111178

[34] CASAlBUONI R., GATTO R., MANNARELLI M. AND NARDULLI G.: arXiv:hep-ph/0201059.

[35] Alford M., Bowers J. A. And RAJAGOPAL K.; Phys. Rev. D, 63, (2001) 074016 arXiv:hep-ph/0008208]; BOWERS J. A., KUNDU J., RAJAGOPAL K. AND SHUSTER E.: Phys. Rev. D, 64, (2001) 014024 arXiv:hep-ph/0101067.

[36] LeiboviCH A. K., RAJAgOPAL K., SHUSteR E.: Phys. Rev. D, 64, (2001) 094005 arXiv:hep-ph/0104073.

[37] RAJAGOPAL K.: Acta Phys. Polon. B, 31, (2000) 3021 arXiv:hepph/0009058; ALFORD M., BOWERS J. A. AND RAJAGOPAL K.: J. Phys. G, 27, (2001) 541 arXiv:hep-ph/0009357.

[38] SCHÄFER T. AND SHURYAK E.: arXiv:nucl-th/0010049; K. RAJAGOPAL K.: in QCD@Work, P. Colangelo and G. Nardulli eds. (AIP, 2001) arXiv:hep-ph/0109135.

[39] DERYAGIN D. V., GRIGORIEV D. YU. AND RUBAKOV V.A.: Int. J. Mod. Phys. A, 7, (1992) 659.

[40] LARKIN A. I. AND OVCHINNIKOV YU. N.: Zh. Eksp. Teor. Fiz., 47, (1964) 1136 ( Sov. Phys. JETP, 20, (1965) 762); FULDE P. AND FERRELL R. A.: Phys. Rev., 135, (1964) A550.

[41] ELIASHBERG G. M.: Zh. Eksp- Teor. Fiz., 34, 735 (1960) (transl. Sov. Phys. JETP 11, (1960) 696.

[42] NAMBU Y. AND JONA LASINIO G., Phys. Rev., 122, (1961) $345 ; \mathbf{1 2 4}$, (1961) 246.

[43] SCHÄFER T.: Phys. Rev. D, 62, (2000) 094007 arXiv:hep-ph/0006034.

[44] BOGOLIUBOV N. N.: Nuovo Cimento 7, (1958) 694; VALATIN J.: Nuovo Cimento, 7, (1958) 843.

[45] SON D. T. AND STEPHANOV M. A.: Phys. Rev. D ,61, (2000) 074012 arXiv:hep-ph/9910491; ibidem 62, (2000) 059902 arXiv:hep$\mathrm{ph} / 0004095$. 
[46] ZAREMBO K.: Phys. Rev. D, 62, (2000) 054003 arXiv:hep-ph/0002123.

[47] RHO M., WIRZBA A. AND ZAHED I.: Phys. Lett. B, 473, (2000) 126 arXiv:hep-ph/9910550.

[48] RHO M., SHURYAK E., WIRZBA A. AND ZAHED I.: Nucl. Phys. A 676, (2000) 273 arXiv:hep-ph/0001104.

[49] HONG D. K., LEE T. AND MIN D.: Phys. Lett. B, 477, (2000) 137 arXiv:hep-ph/9912531.

[50] MANUEL C. AND TYTGAT M. H.: Phys. Lett. B, 479, (2000) 190 arXiv:hep-ph/0001095.

[51] BAlachandran A. P., STERn A. AND TRAhern C. G.: Phys. Rev. D, 19, (1979) 2416; BANDO M., KUGO T. AND YAMAWAKI K.: Phys. Rept., 164, (1988) 217.

[52] EGUCHI T.: Phys. Rev. D. 14, (1976) 2755.

[53] GUSYNIN V.P. AND SHOVKOVY I.A.: arXiv:hep-ph/0108175].

[54] RISCHKE D. H.: Phys. Rev. D, 62, (2000) 034007 arXiv:nuclth/0001040].

[55] RISCHKE D.H., SON D.T. AND STEPHANOV M.A.: Phys. Rev. Lett., 87, (2001) 062001 arXiv:hep-ph/0011379.

[56] OUYED R. AND SANNINO F.: Phys. Lett. B, 511, (2001) 66 arXiv:hep$\mathrm{ph} / 0103168$.

[57] CORNWAlL J. M., JACKIW R. AND TOMBOUliS E.: Phys. Rev. D, 10, (1974) 2428.

[58] CASAlBUONI R., DUAN Z. AND SANNINO F.: Phys. Rev. D, 63, (2001) 114026 arXiv:hep-ph/0011394.

[59] SCHÄFER T. AND WILCZEK F.: Phys. Rev. Lett., 82, (1999) 3956 arXiv:hep-ph/9811473.

[60] SON D. T.: Phys. Rev. D, 59, (1999) 094019 arXiv:hep-ph/9812287.

[61] PISARSKI R. D. AND RISCHKE D. H.: Phys. Rev. D, 61, (2000) 051501 arXiv:hep-ph/9812287.

[62] BARROIS B.: Nonperturbative effects in dense quark matter, preprint UMI 79-04847.mc (1979).

[63] KOGUT J. B., LOMBARDO M. P. AND SINClAIR D. K.: Phys. Rev. $D, \mathbf{5 1},(1995) 1282$ arXiv:hep-lat/9401039.

[64] KOGUT J. B., LOMBARDO M. P. AND SINCLAIR D. K.: Nucl. Phys. Proc. Suppl., 42, (1995) 514 arXiv:hep-lat/9412057. 
[65] SON D. T. AND STEPHANOV M. A.: Phys. Rev. Lett., 86, (2001) 592 arXiv:hep-ph/0005225.

[66] Alford M., KAPUStin A. AND WILCZEK F.: Phys. Rev. D, 59, (1999) 054502 arXiv:hep-lat/9807039.

[67] WONG S. K.: Nuovo Cimento, 65 A, (1970) 689.

[68] HEINZ U.: Phys. Lett. B, 144, (1984) 228.

[69] KELLY P.F., LIU Q., LUCCHESI C. AND MANUEL C.: arXiv:hepph/9406285.

[70] LITIM D. F. AND MANUEL C.: Phys. Rev. Lett., 87, (2001) 052002 arXiv:hep-ph/0103092.

[71] MANUEL C.: Phys. Rev. D, 53, (1996) 5866 arXiv:hep-ph/9512365].

[72] PISARSKI R. D.: Phys. Rev. Lett., 63, (1989) 1129; BRAATEN E. AND PISARSKI R. D.: Nucl. Phys. B, 337, (1990) 569; BLAIZOT J. P. AND IANCU E.: Phys. Rev. Lett., 70, (1993) 3376 arXiv:hep-ph/9301236 and Nucl. Phys. B, 417, (1994) 608 arXiv:hep-ph/9306294.

[73] LIFSHITZ E. AND PITAEVSKII L.: Physical Kinetics, (Pergamon Press, Oxford), 1981.

[74] LOW I. AND MANOHAR A. V.: arXiv:hep-ph/0110285.

[75] SHAPIRO S. L. AND TEUKOLSKI S. A.: Black Holes, White Dwarfs and Neutron Stars: The Physics of Compact Objects, (Wiley-Interscience), 1983.

[76] S. TSURUTA S.: Phys. Rep., 292, (1998) 1; PAGE D.: in The Many Faces of Neutron Stars, R. Bucheri, J. van Paradijs and M. A. Alpar eds. (Kluwer Academic Publishers, Dordrecht) 1998, p. 539.; HEISELBERG H. AND PANDHARIPANDE V.: arXiv:astro-ph/0003276.

[77] CAmeron A. G. W. AND CANUTO V.: Proc. 16th Solvay Conf. on Astrophysics and Gravitation, Universitè de Bruxelles, (1974) p. 221.

[78] GOLD T.: Nature, 221, (1969) 25.

[79] LORIMER D. R.: astro-ph/9911519.

[80] BAADE W. AND ZWICKY F.: Proc. Nat. Acad. Sci., 20, (1934) 255.

[81] COLGATE S. A. AND WHITE R. H.: Astrophys. Journ., 143 (1966) 626; IMSSHENNIK V. S. AND NADYOZHIN D. K.: Sov. Phys. JETP, 36, (1973) 821; BURROWS A. AND LATTIMER J.M.: Astrophys. Journ., 307, (1986) 178; KEIL W. AND JANKA H.T.: Astronomy and Astrophysics, 296, (1995) 145; PONS J. ET AL.: Astrophys. Journ., 513, (1999) 513. 
[82] CARTER G. AND REDDY S.: Phys. Rev. D, 62, (2000) 103002, arXiv:hep-ph/0005228.

[83] PAGE D., PRAKASH M., LATTIMER J. AND STEINER A.: Phys. Rev. Lett., 85, (2000) 2048 arXiv:hep-ph/0005094; BLASCHKE D., KLÄHN T. AND VOSKRESENSKY D. N.: arXiv:astro-ph/9908334.

[84] N. ANDERSSON, Astrophys. J., 502, (1998) 708.

[85] BILDSTEIN L. AND USHOMISRKY G.: arXiv:astro-ph/9911155; ANDERSSON N.: arXiv:astro-ph/0002114.

[86] BODMER A. R.: Phys. Rev D, 4, (1971) 1601.

[87] WITten E.: Phys. Rev D, 30, (1984) 272.

[88] MADSEN J.: Phys. Rev. Lett., 85, (2000) 10 arXiv:astro-ph/9912418.

[89] MADSEN J.: arXiv:astro-ph/0111417].

[90] MADSEN J.: arXiv:hep-ph/0108036].

[91] LINK B., EPSTEIN R. AND LATTIMER J. M.: arXiv:astro$\mathrm{ph} / 0001245$.

[92] CALDWELL R. R. AND FRIEDMAN J. L.: Phys. Lett. B, 264, (1991) 143. 